University of Louisville

ThinkIR: The University of Louisville's Institutional Repository

Electronic Theses and Dissertations

$12-2011$

\title{
The induction of positive affect in a community setting using electronic communications.
}

Philip Andrew Smeltzer

University of Louisville

Follow this and additional works at: https://ir.library.louisville.edu/etd

\section{Recommended Citation}

Smeltzer, Philip Andrew, "The induction of positive affect in a community setting using electronic communications." (2011). Electronic Theses and Dissertations. Paper 1345.

https://doi.org/10.18297/etd/1345

This Doctoral Dissertation is brought to you for free and open access by ThinkIR: The University of Louisville's Institutional Repository. It has been accepted for inclusion in Electronic Theses and Dissertations by an authorized administrator of ThinkIR: The University of Louisville's Institutional Repository. This title appears here courtesy of the author, who has retained all other copyrights. For more information, please contact thinkir@louisville.edu. 


\title{
THE INDUCTION OF POSITIVE AFFECT IN A COMMUNITY SETTING USING ELECTRONIC COMMUNICATIONS
}

\author{
By \\ Philip Andrew Smeltzer \\ B.A. Oregon State University, 1977 \\ M.S. Canisius College, 2001
A Dissertation
Submitted to the Faculty of the
School of Public Health and Information Sciences
In Partial Fulfillment of the Requirements
For the Degree of \\ Doctor of Philosophy \\ Department of Health Promotion and Behavior Science \\ University of Louisville \\ Louisville, Kentucky
}

December 2011 
Copyright 2011 by Philip Andrew Smeltzer

All rights reserved 
THE INDUCTION OF POSITIVE AFFECT

IN A COMMUNITY SETTING

USING ELECTRONIC COMMUNICATIONS

By

Philip Andrew Smeltzer

B.A. Oregon State University, 1977

M.S. Canisius College, 2001

A Dissertation Approved on

November 28, 2011

by the following Dissertation Committee:

Richard Wilson, Dissertation Director

Muriel Harris

Steven McCabe

Barbara Stetson 


\section{ACKNOWLEDGMENTS}

I would like to thank my dissertation chair, Dr. Richard Wilson, for his guidance, mentoring and patience. I would also like to thank my other committee members, Dr. Barbara Stetson, Dr. Steven McCabe and Dr. Muriel Harris, for their insights and suggestions on this document as well as scholarly leadership through various courses. Doctor's Maiying Kong and Timothy Wiemken provided statistical analysis advice that was greatly appreciated. I would like to acknowledge my wife Emily for her patience, encouragement and understanding throughout my academic journey. She has sacrificed much in support of my studies through the years beginning in Buffalo and concluding here in Louisville. Also, thanks to all members of my family including sons, daughters, brother and sisters. A special mention to previous doctors in the family who provided inspiration and life lessons including Mary A. Moffitt Ph.D., the late Warren Henry Smeltzer D.D.S., Warren Henry Smeltzer Jr. D.V.M., and Mary K. Smeltzer (Rice). 


\title{
ABSTRACT \\ THE INDUCTION OF POSITIVE AFFECT \\ IN A COMMUNITY SETTING \\ USING ELECTRONIC COMMUNICATIONS
}

\author{
Philip A. Smeltzer
}

November 28, 2011

Objective: To evaluate the influence of positive affect inductions on daily and weekly Positive and Negative Affect Scales (PANAS) in a community setting.

Methods: Humor messages were distributed to participants four times per day during a treatment week with general health messages distributed in the crossover week. Subjects completed a series of self-report psychosocial and behavioral survey instruments on a weekly basis and daily PANAS responses. Messages were delivered through either email or SMS/text messages.

Results: The daily and weekly PANAS scores were higher during treatment periods compared to the neutral periods. The observed trends were suggestive of successful positive affect induction although the statistical analysis did not indicate statistically significant group differences.

Conclusions: The induction of positive affect manipulations in a community setting appears to have potential. Future research with larger cohorts and refined methods to limit missing data may lead to further insights on positive 
affect influences. There are indications that positive affect may be increased through humor messages in an applied setting. 
TABLE OF CONTENTS

PAGE

ACKNOWLEDGMENTS

ABSTRACT

LIST OF TABLES

LIST OF FIGURES

iii

iv

ix

$x$

INTRODUCTION

$\begin{array}{lc}\text { Hypotheses } & 6\end{array}$

$\begin{array}{ll}\text { Delimitations } & 7\end{array}$

$\begin{array}{lr}\text { Limitations } & 8\end{array}$

Operational Definitions $\quad 8$

REVIEW OF LITERATURE

$\begin{array}{ll}\text { Introduction } & 12\end{array}$

Definitions 13

$\begin{array}{lr}\text { Overview } & 16\end{array}$

$\begin{array}{ll}\text { Physical, Cognitive, Neural Evidence } & 19\end{array}$

$\begin{array}{ll}\text { Theoretical Models } & 22\end{array}$

$\begin{array}{ll}\text { Measurement Challenges } & 26\end{array}$

Positive Affect Impact $\quad 30$

$\begin{array}{ll}\text { Health Impact of Positive Affect } & 39\end{array}$

$\begin{array}{ll}\text { Conclusion } & 46\end{array}$

$\begin{array}{ll}\text { Positive Affect Induction } & 48\end{array}$ 
Research Needs and Problems

METHODS AND MATERIALS

Research Problems $\quad 51$

Research Design and Methods $\quad 52$

Study Participants 53

$\begin{array}{ll}\text { Participant Screening } & 54\end{array}$

Pilot Validation Study and Methodology 55

$\begin{array}{ll}\text { Randomization } & 59\end{array}$

$\begin{array}{ll}\text { Participant Selection Criteria } & 61\end{array}$

$\begin{array}{ll}\text { Limitations, Delimitations } & 62\end{array}$

$\begin{array}{ll}\text { Study Design } & 62\end{array}$

Measurement Methods and Instrumentation 65

$\begin{array}{ll}\text { Measurement Challenges } & 77\end{array}$

$\begin{array}{ll}\text { Statistical Analysis Methodology } & 79\end{array}$

$\begin{array}{ll}\text { Outcome Measures } & 82\end{array}$

Safety Issues $\quad 84$

$\begin{array}{ll}\text { Study Closeout } & 87\end{array}$

$\begin{array}{ll}\text { Data Safety Monitoring Board } & 87\end{array}$

$\begin{array}{ll}\text { Benefits } & 87\end{array}$

Blinding, Unblinding, Masking $\quad 88$

Exploratory Study Summary $\quad 89$ 
MAIN STUDY RESULTS

Introduction

Participants

$\begin{array}{ll}\text { Research Questions } & 97\end{array}$

$\begin{array}{ll}\text { Results } & 98\end{array}$

$\begin{array}{ll}\text { Summary } & 107\end{array}$

SUMMARY, CONCLUSIONS, RECOMMENDATIONS

$\begin{array}{ll}\text { Introduction } & 108\end{array}$

$\begin{array}{ll}\text { Research Needs and Problems } & 110\end{array}$

$\begin{array}{ll}\text { Conclusions } & 111\end{array}$

$\begin{array}{ll}\text { Discussion } & 113\end{array}$

$\begin{array}{ll}\text { Recommendations } & 118\end{array}$

$\begin{array}{ll}\text { REFERENCES } & 121\end{array}$

APPENDICES

1. Participant Recruitment Flyer $\quad 130$

2. Humor Treatment Message Samples 131

3. Neutral Treatment, General Health Message Samples 133

4. Positive and Negative Affect Scale Survey Instrument 135

5. Pilot Study Summary 136

$\begin{array}{ll}\text { CURRICULUM VITAE } & 159\end{array}$ 


\section{LIST OF TABLES}

TABLE

PAGE

1. Positive Affect Measurement Methods

2. Positive Affect Induction Methods 29

3. Summary of Positive Affect Studies 38

4. Demographics 96

5. Cohort Characteristics 96

$\begin{array}{ll}\text { 6. Daily PANAS Scores } & 100\end{array}$

7. Seven-Day Recall PANAS Scores 100

8. Seven-Day Recall SF-36 Emotional Scales 102

9. Growth Curve Outputs 106 


\section{LIST OF FIGURES}

FIGURE PAGE

$\begin{array}{ll}\text { 1. Affect as Information } & 18\end{array}$

2. Affect Infusion Model 25

3. Emotion Induced Eating 46

4. Cohort Tree Diagram 60

5. Study Design and Methodology 65

6. Telephone Call Interaction Flow Chart 76

7. Cohort Study Diagram - Main Study 95

$\begin{array}{ll}\text { 8. Individual PANAS Score Plots } & 101\end{array}$

9. Treatment Group 1 and Treatment Group 2 Daily PANAS Scores 102 


\section{CHAPTER I \\ INTRODUCTION}

Public health issues in the United States over the last 100 years have moved from primarily communicable diseases to increased emphasis on healthy lifestyles and personal habits (USDHHS 2001). Lifestyle behaviors such as sedentary habits, poor nutritional intake and excess body fat have been identified as key factors in the development of chronic conditions including heart disease and cancer (Finkelstein, Fiebelkorn et al. 2003; Schroeder 2007). Behavioral change research has evolved in theories and constructs targeting lifestyle changes. There appear to be multiple theories, cognitive and behavioral techniques that have been studied in applied settings (Glanz, Rimer et al. 2002). The literature is informative regarding these theories and successful applications, yet the prevalence of lifestyle issues and chronic conditions continue to increase (USDHHS 2000).

The use of technology such as the Internet and phone-based counseling has been documented in the literature as effective and promising (Wing, Jeffery et al. 1996; Tate, Wing et al. 2001; Castro and King 2002; Harvey-Berino, Pintauro et al. 2002; Smith, Cameron et al. 2004; Vanwormer, Boucher et al. 
2006; Eakin, Lawler et al. 2007; Booth, Nowson et al. 2008; Estabrooks and Smith-Ray 2008; Hunter, Peterson et al. 2008; Tucker, Cook et al. 2008). An advantage of phone or Internet based interventions is the ability to increase efficiency and subsequently scale up to larger population based programs (Vanwormer, Boucher et al. 2006). Phone and Internet interaction has proven effective (Tate, Wing et al. 2001; Tate, Jackvony et al. 2006; Hunter, Peterson et al. 2008) and increases the number of interactions per resource. A drawback has been the percentage of a target population who access the Internet or phone resources without promotional efforts such as prompting. The utility of in-person counseling, Internet interventions, or phone-based interactions, all appear to have merit in changing behavior.

The emergence of the positive psychology field and the influence of positive and negative emotions on health behavior has begun to appear in the literature and is cited as a research area within the National Institute of Health (Fredrickson 2001; Seligman, Steen et al. 2005). The potential impact on health through improved application of behavior change techniques through positive emotion or positive affect treatments may have merit (Isen 2001; Macht 2008; Andrade and Ariely 2009). The use of proven techniques in combination with useful behavioral theories has the potential to improve lifestyles in targeted populations. The impact of these population based lifestyle improvements can result in improved health status and possibly reduced medical expenditures. This backdrop of needs for lifestyle changes across the U.S. population combined with national issues of increased chronic condition prevalence and 
related health care costs, support the investigation of new techniques to influence behavior change in individuals. Specifically, this proposed study will study the impact of positive affect induction in an applied setting.

Positive affect induction has not been widely studied in applied settings in a prospective randomized clinical trial design. Although positive affect and positive emotions are associated with healthier behaviors and increased health status, the temporal relationship and latency between the induction of positive affect and subsequent health behavior decisions has not been widely reported. The research implications and opportunities to explore the impact of positive affect on health care decisions and behavior appear to be numerous (Macht 2008). Meller's and McGraw's subjective expected pleasure theory has not been applied to health and medical decision making situations (Mellers and McGraw 2001). The role of acute emotions has not been applied to personal decisions regarding medical care and preventive care (Diffenbach, Miller et al. 2008), and is recommended for additional study of nutritional intake (Macht and Simons 2000). It appears that depressed mood supports indecisiveness, yet the impact of this circumstance on health related decisions has not been directly explored (Consedine 2008). The effects of specific emotions such as anger, sadness and fear has not been reported (Macht 2008). It appears that evidence is absent regarding the effect of positive affect on coping behavior and health behaviors (Lyubomirsky, King et al. 2005).

A review of the literature outlined the similarities and differences between mood, emotion and affect (Watson, Wiese et al. 1999; Fredrickson 2001; 
Consedine 2008). Emotion and affect have been reported through multiple studies cited to influence the decision making process (Isen and Levin 1972; Isen, Shalker et al. 1978; Isen, Daubman et al. 1987; Isen 2001; Isen 2008). Emotion interacting with decision making processes has been labeled an emotional schema by Izard (Izard 2009). This interwoven relationship between cognitive processes and emotional influence is a central aspect of examining the latent and temporal aspects between positive affect and health care decisions. Specifically, the focus of the reviewed studies in the following sections is on positive affect. The influence of positive affect on cognitive processes is evidenced in diverse tasks such as product evaluations; puzzle completions, word associations, personality appraisals and health related decisions. The extreme ranges of positive affect were not as effective as moderate affect on improving performance in these tasks (Isen 2000; Roehm and Roehm 2005; Isen 2008). Light to moderate inducement of positive affect has been shown across this range of tasks to improve the decision-making process and task outcomes. These findings can be used to compliment current behavioral change interventions. The potential implications are an increased effectiveness of behavioral changes using positive affect.

When positive affect is induced the resulting effect is specific and not a broadcast influence. The application of positive affect requires targeted use to increase the impact and yield desired results. Increasing positive emotional level can be driven by the cueing of positive affect and an upward spiral of emotions as more positive cues are recognized and this upward cycle begins to perpetuate 
(Fredrickson 2001). Positive affect is not a panacea for transforming clinical depression or high negativity moods or emotions within individuals. Individuals may express positive emotions on a given topic and be in a strong negative state for other issues. The affect infusion model (AIM) outlines a framework where positive affect is processed as information within the cognitive process (Forgas 1995). The cognitive process for a given issue may not tap into the positive affect when present, and thus the selectivity and treatment of affect as a data packet or information can describe the interaction of positive affect within decision making.

The study of cerebral processes involving cognition and emotion lend additional insight to the physical explanation for causal factors of positive affect. There is evidence revealing the influence of dopamine on activation patterns and neural firings enhances positive affect, the conclusion that positive emotions are treated as information within cognitive decision-making processes (Ashby, Isen et al. 1999; Dreisbach and Goschke 2004).

The approach to health related cognitions and behavior change through positive emotions is a relatively recent field of study compared to the studies around negative affect and the therapeutic value of cognitive behavioral therapy. The experiential research concludes that positive affect improves creativity, problem solving, innovation, social interaction and task motivation (Isen and Levin 1972; Isen, Shalker et al. 1978; Isen, Daubman et al. 1987; Isen, Nygren et al. 1988; Kahn and Isen 1993; Isen 2001). The implication of incorporating this influence into health behavior interventions has promising potential. A few recent 
studies have begun to explore this aspect of positive affect influence on health decisions. Similar to the processes associated with the transtheoretical model (Prochaska, Velicer et al. 1994), or the influence of self-efficacy on behaviors (Bandura 2001), the use of positive affect has potential to become a component in behavioral change models.

\section{The Questions:}

Can positive affect be induced in an applied setting across multiple days, comparing treatment and control groups?

A secondary research question is, will the induction of positive affect through a telephone based interactive voice response system (IVR) increase positive affect in the treatment group compared to a control group? The instrument to assess this change is the PANAS.

Another secondary research question framed becomes, will the induction of daily positive affect be an effective means of driving an increase in daily physical activity in participants in the treatment group compared to a control group in a community environment

\section{Hypotheses}

Ho-1: Patients who receive positive affect inductions will not increase their daily positive affect scores on a PANAS scale instrument.

Ho-2: Patients who are targeted for physical activity and receive positive affect induction will not yield a change in daily physical activity compared to comparable sets of control subjects. 


\section{Delimitations}

Delimitations for the proposed study include a maximum distance of 20 miles between a study site and geographical residence, pre-menopausal females, BMI $27 \mathrm{~kg} / \mathrm{m}^{2}$ to $35 \mathrm{~kg} / \mathrm{m}^{2}$, no confounding medical conditions. The distance between study site and residence is designed to decrease the cohort erosion from missed appointments. Sufficient volunteers are expected to be available within the urban boundaries of Louisville for the study. Emotional states, perceptions regarding exercise and prevalence of depression have been documented as areas that differ when comparing males vs. females. The delimitation of female participants is intended to reduce variability between participants and a potential confounding factor. The pre-menopausal criterion is designed to create a homogeneous sampling frame that excludes hormonal influence on emotions, typically noted during menopause. Depression and other negative emotional traits have been documented in the severely obese (Stunkard 2003; Simon, Ludman et al. 2008). The limit on body mass index from mid-range overweight to mid-range obesity is designed to elicit a population that has increased need for lifestyle changes, but not severe behavioral health issues. Medical conditions such as psychosis, suicidal ideation, depressive symptomology, pregnancy and physical limitations to mobility are criteria for exclusion. The medical factors are identified as probable confounding issues that have the potential to invalidate outcomes and findings. 


\section{Limitations}

The validity of positive affect induction through a phone interactive voice response system has not been established. The treatment effect of a multiple induction regimen including positive affect across several days has not been reported in the literature. The availability of cell phone network access, quality of phone communications and potential malfunction of the computer generated call system may limit the effectiveness of intended treatments. The participant willingness to accept and listen to scheduled calls is a potential limitation. Applied setting circumstances such as a massive failure of the cellular phone system as a result of a natural disaster or catastrophic system failure are potential limitations. Any of these factors may impede the treatments designed for delivery through the phone system.

\section{Assumptions}

Participants will engage in the phone-based interventions and listen to the full messages provided in the calls. The self-reported psychological, social and behavioral responses to survey instruments at baseline and final outcomes will be completed accurately and with honesty by participants.

\section{Operational Definitions}

\section{IVR - Interactive Voice Response}

IVR, interactive voice response is the label used to describe a computer driven telephone system that most commonly initiates a telephone call with a specific individual and engages the participant in a series of questions and

responses that allows the individual to tailor the content of the conversation. This 
technology is well suited for surveys, data collection and tailored conversations with the respondent (Delichatsios, Friedman et al. 2001). The IVR technology is the primary communication channel designed to induce positive affect in participants for the proposed study.

\section{Medical Provider}

The term medical provider is defined in this document as an individual who provides medical care to a participant. Although this term is commonly used as a synonym for physician, this term will be used in the more general definition of medical provider to include physician assistants, nurse practitioners, and physicians. Affiliated medical professionals such as exercise physiologists, nurses, and registered dieticians are not defined as medical providers within this study

\section{Positive Affect}

Positive affect is defined in this study as an emotional state that is transitory rather than dispositional. Positive affect is present when a participant expresses positive emotions such as happiness, joy, feeling good, pleasure (Isen 1999; Isen 2008).

\section{Positive Affect Induction}

Positive affect can be induced for an individual through the administration of small unexpected gifts, an uplifting film clip, and internalization of affirmations, cognitive recall of a pleasant autobiographical experience - typically through a meditative state, humor or sensory stimulation of a positive memory experience such as smell or taste. Several of these positive affect induction techniques will 
be used in the study and are referred to as positive affect induction (Isen, Daubman et al. 1987; Lyubomirsky, King et al. 2005; Pressman and Cohen 2005).

\section{Crossover Study Design}

The research methodology followed in this study uses a three-treatment group model. The first treatment group will include the positive affect induction call during the first period and then a neutral call the second period. The second treatment group will start with the neutral call and then crossover to the positive affect intervention in the second period. The switching of treatments or crossover between these two groups establishes the label of a crossover design. The third treatment group receives usual care for the duration of the treatment periods.

\section{Telephony, Telehealth or Health Dialog System}

The combination of an integrated phone system with a computer to interact with an individual is operationally defined as a telephony system (Castro and King 2002). The IVR technology or interactive system is considered synonymous for the purposes defined in this paper. Telephony systems as described elsewhere may not include the IVR capability used in this study, but may describe an integrated use of telephones with computer dialing, computer assisted answering and telephone routing. The use of a telephone in an intervention communication channel has also been referred to as a health dialog system (Bickmore and Giorgino 2006). 


\section{Pilot, Exploratory, Preliminary or Validation Study}

Prior to the major study proposed a smaller study is to be conducted. A qualitative study of induction techniques will be completed. This preliminary study is designed to empirically study the ability to induce positive affect in a participant group. The conclusions and findings of the pilot study may be used to modify the primary study methodology. 


\section{CHAPTER II \\ LITERATURE REVIEW}

\section{Literature Review Introduction}

A review of applicable literature within the defined boundaries of positive affect and subsequent influence of health follows. Multiple search strategies were employed to obtain a comprehensive review of published research studies. Online databases available through a university library system (OVID, Medline, PubMed, PsychINFO, ProQuest Dissertation Database) were searched, using key words including positive affect, positive emotions, negative affect, positive mood, negative mood. Each paper identified as applicable was reviewed and the ancestry method was used to comb each paper's reference list for both articles and periodical titles. Additional articles were identified through this method as well as a review of journal editions for the period 2004-2009. The final body of literature reviewed and cited in this study includes more than 140 papers, books and book chapters.

The review is organized into nine primary sections. The first two sections provide operational definitions of emotions, moods, and affect, followed by a review of general implications and a physiological basis for positive affect.

Theoretical models of positive affect are then reviewed, as well as measurement challenges for consideration. Next, the impacts of positive affect in general and then specifically how positive affect influences personal health decision-making. 
The implications and conclusions drawn from the literature are presented, followed by potential research needs and problems.

\section{Definitions}

Positive emotions are tangible feelings that are derived from a personally relevant situation. These emotions are relatively shorter in time expression compared to moods and can be drawn from our conscious awareness (Consedine 2008). Emotions are linked to a personally relevant experience or issue, and are expressed in the foreground of consciousness. The emotion is typically initiated when an individual forms an appraisal of a relevant event. The appraisal may be conscious or unconscious and begins a chain of responses that can be elicited across multiple physical expressions (Fredrickson 2001). An emotional state provides for physiological responses such as heart rate, blood pressure, muscle tension, hormonal release, facial expression, cognitive processing and attention focus. These physiological changes can occur almost instantly as an emotional state is induced (Consedine 2008).

All emotions have common as well as distinct characteristics. Interpersonal reactions and animal to human events can elicit emotions. The human interaction with inanimate stimuli such as music, weather, and loss of interpersonal communication can also provide strong emotional feelings. Emotions are unique as antecedent triggers stimulate them, create various physiologic responses and possess certain strength. Emotions are the product of an external trigger or environment. 
The temporal relationship between triggers and negative emotions is more distinct than triggers that drive positive emotions. A negative emotional trigger such as a loud noise, an angry dog or sad news will typically elicit emotional reactions that are immediate and directly related to the trigger. Negative emotions are many times triggered by events or instances that threaten well being (Isen 1999; Fredrickson 2005; Fredrickson and Cohn 2008). Thus the reactions to these triggers are rapid and typically include physical actions such as running, crying or a defensive posture (Zajonc 1980). Positive emotions are many times elicited through more subtle triggers. Examples of positive emotional triggers include humorous joke, an unexpected piece of good news, an unexpected compliment, a financial gift (Isen 1999). The relationship between negative emotional triggers and subsequent feelings is more direct. Positive emotional triggers are not as direct and may not be easily identified by the individual when queried (Isen and Erez 2007).

Emotions are not volitional states that can be willed. All emotions are noted by their short duration, almost instantaneous appearance, involuntary occurrence and consistent application (Ekman 1992). Emotions are commonly categorized into discrete compartments such as fear, interest, gratitude, contentment, pride, love, joy, anger (Fredrickson 2001).

Biological needs that are fulfilled can create a sense of pleasure such as sexual gratification, satiation of thirst or hunger. This pleasurable subjective feeling is comparable to positive emotions (Fredrickson 2005). The distinction between a physical sensation and an emotion is the cognitive assessment that is 
required to appraise an emotional state. The stimulation of physical senses dominates pleasure feelings, but is not typically either found or required to drive an emotion (Fredrickson and Cohn 2008). An emotional state can be described by strength and by category; intense fear, mild interest, overwhelming joy (Watson, Wiese et al. 1999). Emotions create psycho physiological changes in responses to a stimulus or change in the surroundings. Emotional states can dominate our consciousness. Emotions have evolved as a fast, efficient response to an external stimulus. Emotions force a conscious decision to react to the emotional trigger or stimulus. Emotions can move to the foreground of consciousness, dictating a decision on action. Moods and affect occupy a less conscious state of awareness (Rosenberg 1998; Izard 2009). The cognitive process is influenced by the affect, yet the affect is not available at a conscious level of recall. These emotional states are created through a direct circuit in the thalamus and amygdala bypassing cortical involvement (LeDoux 1996).

Moods are relatively longer in duration compared to emotions and are not as often derived from a specific circumstance. A mood is not as obvious and not easily drawn from consciousness (Rosenberg 1998). A mood is more of a disposition compared to an emotion as more transitory. Moods are not created or aroused by an antecedent event (Forgas 1994). This dispositional state is also stable. A mood can be expected to remain intact, until a change or modifying factor is infused (Erber and Erber 2000).

Affect is a more general construct that passes through emotions, sensations, attitudes, and moods. Affect refers to feelings that are accessible 
during cognition (Fredrickson 2001). The influence of affect is more general and not as anchored to a specific circumstance when compared to emotions (Ryff and Singer 1998; Russell and Carroll 1999), although affect can be induced through exposure to a specific trigger. Affect has been induced through a specific event or incident across a variety of settings, both positive and negative. Successful affect inductions have been reported using film clips, unexpected gifts and autobiographical recall (Isen and Erez 2007; Isen 2008). Affect is distinguishable as a longer lasting influence compared to emotion, but is typically defined as more transitory than a mood. Moods are more commonly categorized as a dispositional state (Ekman 1992; Forgas 1995; Fredrickson 1998; Keltner and Gross 1999; Isen and Erez 2007; Fredrickson and Cohn 2008; Isen 2008). Affect is also characterized as bipolar in dimension, positive or negative rather than discrete categories (Watson, Wiese et al. 1999; Barrett, Gross et al. 2001). Slovic and Peters define affect as "a faint whisper of emotion and the specific quality of positive or negative that, with or without consciousness, is related to a positive or negative quality of the circumstance" (Slovic, Peters et al. 2005). Positive affect has similarly been defined as a "mild increase in positive feelings brought about by commonplace, everyday events" (Isen 1999).

\section{Overview}

Positive affect can be induced through an external influence. The topic or issue that is the target of the influence must be viewed as neutral or in a positive perspective for the induction to be effective. Positive affect has not been proven to reverse a negative emotion or feeling. Positive affect appears to increase the 
positive valence when the issue of interest is viewed as positive or at a minimum neutral (Isen 2005; Isen 2008). This specificity of effect is a key aspect of positive affect. This specificity is not a mood shift that acts as a consistent bias, but a trigger than only effects certain issues (Isen 1999). Emotions and cognitions appear to be bidirectional causes and effects, based upon the trigger event. That is, an emotion can directly influence the cognitive process or the cognitive evaluation of a situation or, conversely, the environment can result in a changed emotional state (Isen 2008). Zajonc posited emotions as a major influence in decision-making. Emotional reactions and the route of activation in the brain often precede any cognitive reaction to certain events, preceding the initial cognitive or physical response. Our personal-initial interpretation of an event or an object may be an automatic response of emotions (Zajonc 1980; Zajonc 1984). Emotional heuristics or shortcuts may be associated with memory events or stimulus. An individual will access this heuristic when the trigger or memory event is encountered and thus produce an influence in the decision process (Finucane, Alhakami et al. 2000). Affect can overwhelm cognition and dominate the decision making process in certain circumstances where temptation for immediate gratification can diverge from more distal risk considerations (Lawton, Conner et al. 2009).

Affect can be viewed as information, not just an influence of valence. Affect would then be included as data used in the decision-making process including risk perceptions (Diffenbach, Miller et al. 2008). The affect as information model is depicted in Figure 1. This flow chart model outlines several 
of the concepts reviewed in the literature. The chart outlines how affect is treated as both new information and as a subsequent influence on future behaviors. The feedback loop effect illustrated by Diffenback, Miller et al. seems to capture the essence of how affect is both information for current decisions as well as a stored packet of information or memory that can be recalled in future cognitive processes. Affect as information can be processed as useful input, similar to currency. Affect can also be used to highlight or spotlight the affective input or other information. When new information is identified, this affective data is available for context and influence for subsequent cognitive processes and affective judgments.

Figure 1. Affect as Information

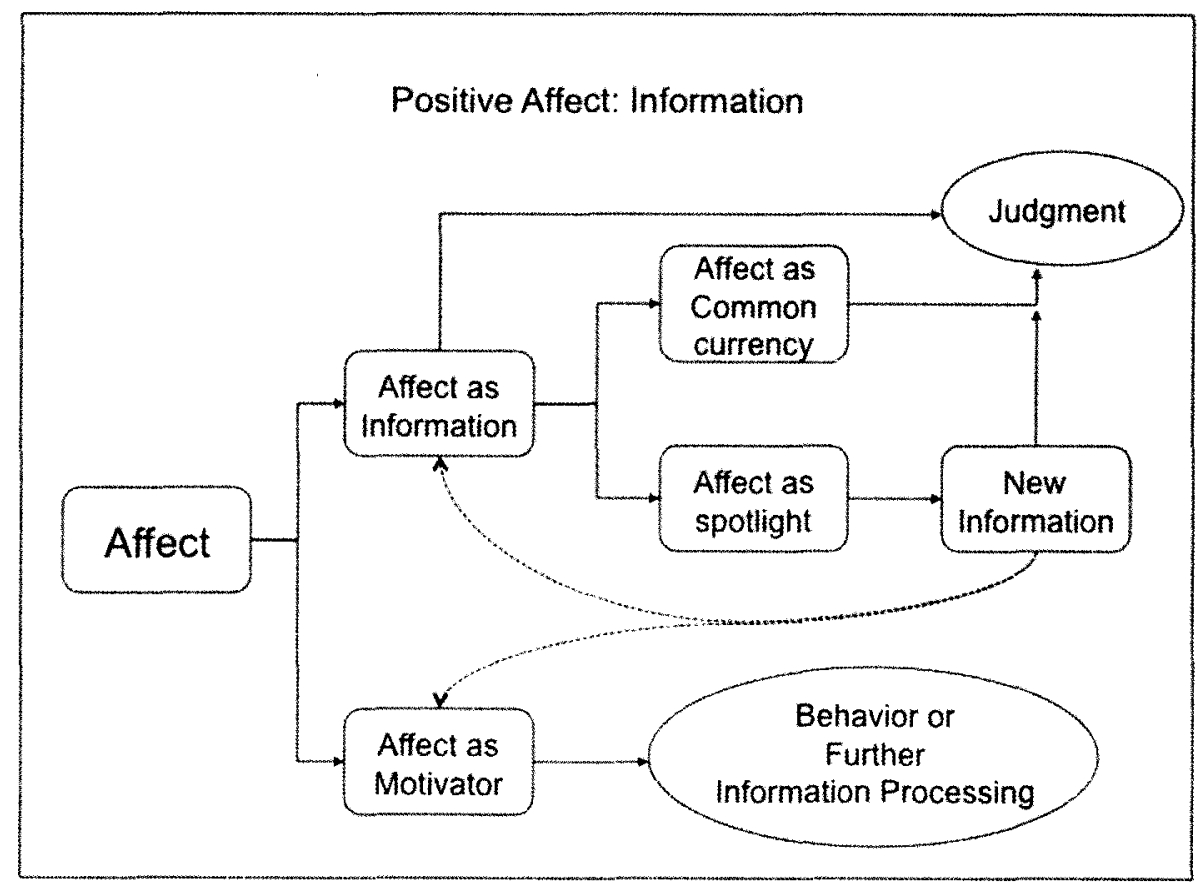

Adapted from (Diffenbach, Miller et al. 2008)

The review of current behavior change models and subsequent theories and construct discussion lack the consideration of emotions as part of any model 
(Diffenbach, Miller et al. 2008). Positive affect may provide a cue for decision making points, which may impact the evaluation of probabilities and the likelihood of a specific scenario occurring (Zajonc 1980; Slovic, Peters et al. 2005). Affect serves as an additional packet of information in a cognitive process, guiding judgments along the decision-making path. Thus, a positive affect influences the consideration of pro's and con's for any given decision point (Diffenbach, Miller et al. 2008). When an individual is in a more positive state of emotion or affect, more positive cues from memory are available. Positive information stored in memory is diverse and extensive and produces a broader, more complex packet of ideas and considerations. This richer and fuller set of information leads to likely solutions that are creative and innovative as a result of this positive affective state (Isen 1999). Study participants in a positive mood try harder, are more motivated, perform tasks better and stay on task longer compared to individuals in a neutral mood (Erez and Isen 2002).

\section{Physical, Cognitive and Neural Evidence}

The literature is informative regarding the physical, chemical and neurotransmitter processes between emotions as they develop and interact with cognitive processing. Different emotion states such as joy, anger, sadness and others have been mapped to unique neural patterns that support the influence of emotion and affect on decision making and behavior (Damasio, Grabowski et al. 2000). The activation of various regions in the brain have been associated with specific emotions (Ashby, Isen et al. 1999). These conclusions provide a 
physiological basis for the influence of positive affect on behavior and the decision making process.

The neurotransmitter and chemical explanation for the induction of positive affect has been linked to the release of dopamine. Unexpected reward or signals of an anticipated reward cause dopamine release in animals. The frontal cortical brain sectors contain dopamine projections. These same sectors that include dopamine influence, the anterior cingulate cortex, are associated with cerebral processes that include thinking and working memory (Ashby, Isen et al. 1999). Increased emotional states can influence other physiological responses. Immunological system responses were activated when mood states were heightened, both positive affect and other emotions. Heart rates, T-cell counts, cortisol levels and physical movement were all impacted by longer term moods (Futterman, Kemeny et al. 1994). There is evidence that increased brain dopamine associated with positive affect improves cognitive flexibility (Dreisbach and Goschke 2004). The stimulation and subsequent release of dopamine when an unexpected gift is introduced can last in excess of 30 minutes, yet an anticipated reward seems to stimulate the firing of dopamine for as few as 10 seconds (Ashby, Isen et al. 1999).

Positive affective states trigger memories relating to positive events. The positive affective state does not influence memories that are categorized as negative material or negative memory packets (Isen 1999). Positive emotions open the door and allow us to explore potential opportunities, as opposed to negative emotions that have evolved as more protective in nature, typically in 
response to a threat (Lyubomirsky, King et al. 2005; Fredrickson and Cohn 2008). Affect has been defined as stemming from one of two unique functional stimuli, either integral or incidental. Integral affect is linked to a stimulus that results in experienced emotion, and the focus of interest in this literature review. Incidental affect, which is not the focus of this study, are emotions that are independent of any stimulus (Peters, Vastfjall et al. 2006),.

Individuals who exhibit dominant extraversion traits report increased cheerfulness, boldness, self-assurance, enthusiasm and energy compared to introverts. The relationship between the positive affectivity and social activity is bidirectional. The extraversion increases positive emotionality and the feelings of cheerfulness, liveliness and enthusiasm are associated with a desire for more interpersonal contact. Positive affectivity is both a cause and an effect of social behavior (Watson 2005). Rosenberg summarized six studies that reported a correlation between self-reported personality traits such as extraversion and neuroticism and the emotion scores after an induction treatment. The induction of positive affect was reported to be more profound in extraverts, compared to introverts (Rosenberg 1998). Positive affect when induced in a work group decreases antagonism and conflict through both interpersonal and intergroup impact (Dovidio, Gaertner et al. 1998). Positive affect can be promoted through positive social support, such as healthy behavior practice or reducing barriers to change with a partner who serves as the social control agent. A social control agent is an actor who serves as a central figure who influences another individual. Social control agents have been identified as spouses, friends, 
medical providers or celebrities. The positive affect through social support appears to have a greater influence on women than men (Tucker, Orlando et al. 2006).

\section{Theoretical Models of Positive Affect}

Fredrickson's broaden-and-build theory is a model of emotion designed to outline the unique aspects of positive emotion rather than the previous models of emotion that were dominated by negative considerations (Fredrickson 1998).

The broaden-and-build theory posits that positive emotions are exhibited through "broadened thought-action repertoires", and then describes the functional impact as the construction of long-lasting assets for personal use in the future (Fredrickson 1998; Fredrickson 2001). The broaden component of the broadenand-build theory explains that positive emotions increase the number of thoughtaction options or broaden the momentary repertoire for the individual to access (Fredrickson and Branigan 2005). These transitory states of positive emotions begin to build over time. The accumulation and increase of broadened range of actions provides new resources for the individual and constitutes the buildingeffect of the broaden-and-build theory (Fredrickson and Branigan 2005). The broaden and build theory is supported by the findings of Isen and Kahn, where positive affect induction resulted in more creative cognitive processes and increased consideration of alternate decisions (Isen, Daubman et al. 1987; Kahn and Isen 1993). This broaden-and-build model is positioned as a lasting effect on future behavior. The positive memory repertoires are available to be 
extracted during future positive emotional states and begin another cycle of broaden-and-build.

An examination of the broaden-and-build theory was completed on a treatment group over an eight-week period. Participants were trained in meditation. Treatment group participants meditated daily with specific focus on kindness and love. The treatment group participants began reporting higher levels of positive emotions compared to a wait-list control group at the three week mark (Fredrickson and Branigan 2005). The increase in positive emotions and broadened cognitive processes become predictive of future increased positive affect levels. The end result of these upward and increasing spirals of positivity is a richer and deeper set of coping resources when faced with future challenges (Fredrickson and Joiner 2002). This component of the broaden-andbuild theory helps account for long-lasting impact on thoughts and actions that results from a short-lived successful positive affect induction (Fredrickson and Branigan 2005).

A second theoretical model to explain the influence of positive affect is the affect infusion model (AIM). This model outlined by Forgas defines the process where affectively loaded information is introduced and then influences the subsequent judgments and decisions of the individual. This process is incorporated into the judge's considerations and eventually influences the end point decision or judgmental outcome (Forgas 1995).

AIM outlines four routes of judgment; direct access, motivation, heuristic, substantive strategy, summarized below. The direct access strategy extracts 
previously stored judgments that are strong and firmly formed. Direct access is employed when the topic of interest is well known. The motivation processing strategy selectively extracts judgments that will support a desired outcome. The goal in motivation processing is to reach an accurate appraisal of the topic of interest or target. Use of heuristic processing is an efficient use of limited information to form a judgment. The use of heuristic processing is likely when the target topic is well known without strong personal relevance and the availability of cognitive processing capacity is limited. When the substantive processing strategy is employed, the individual selects novel information on the target topic and relates this information to pre-existing information. The strategy of substantive processing is more likely when the target topic is complex or unfamiliar. The latter two strategies of substantive and heuristic processing are thought to allow for more avenues of affect infusion (Forgas 1994).

The selection of a judgment route is influenced by: the actual decision target, the judge or decision-maker and the environmental context that surrounds the target and judge to provide situational consideration (Forgas 1995). Mood effects on judgments can be expected when either affect-priming, constructive processing or heuristic processing are the major mechanisms of affect infusion (Bower 1999; Forgas and Bower 1988; Schwarz 1990 in (Forgas 1994)). As information is processed during the judgment and decision forming phase, the influence of affect increases.

The flow of information processed during judgments is summarized in figure 2. The environmental context from the task at hand, the state of the judge 
or person and various situational issues are processed. The substantive and heuristic routes on the left side produce the highest affect influence. Motivated processing and direct access on the right side of the figure are the decision routes least influenced by affect.

Figure 2. (Forgas 1995)

\section{The Affect Infusion Model (AIM)}

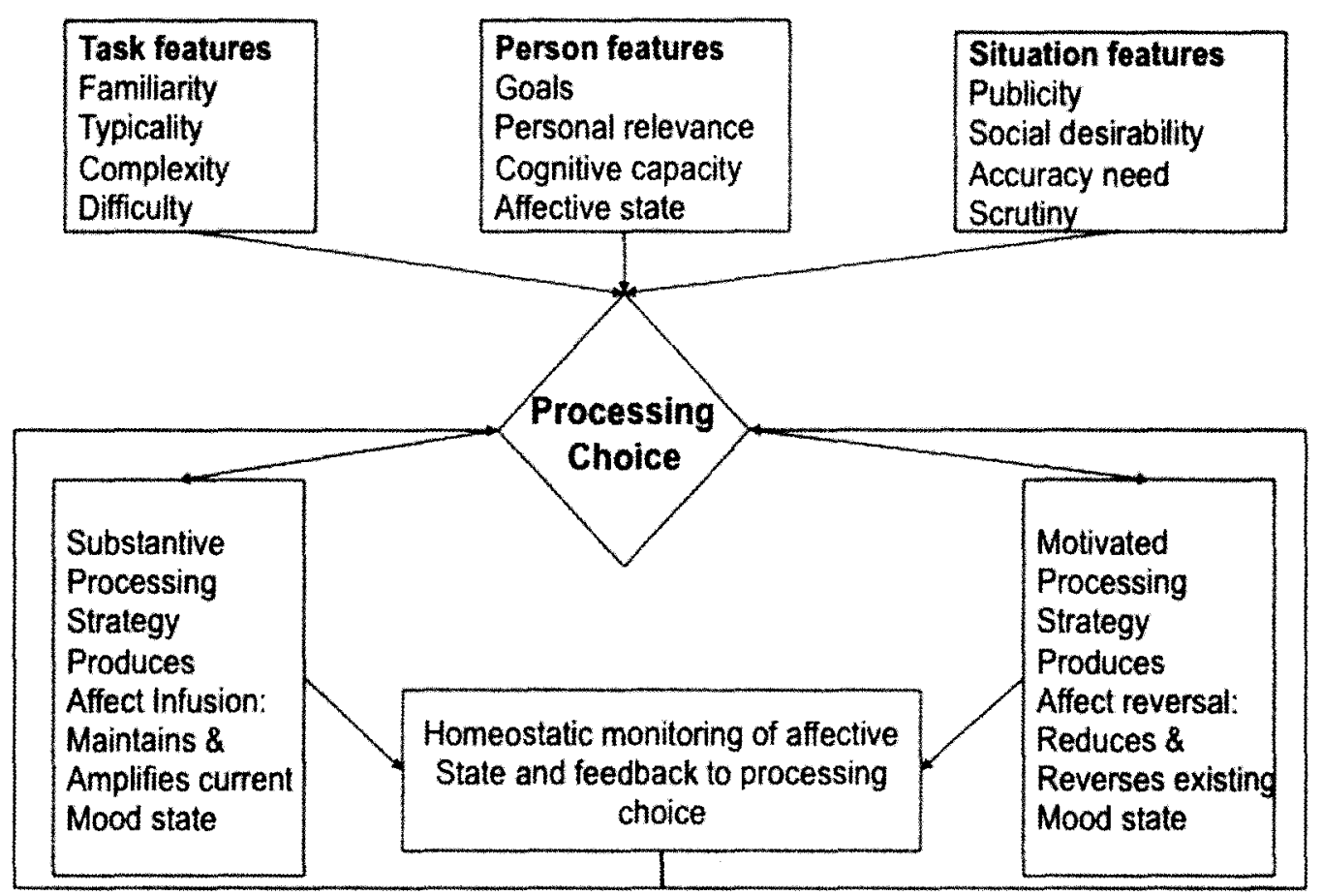

Positive affect can serve as a cue for the selective use of information in the constructive processing of judgments. This results in the selection, attention, encoding and retrieval of congruent information as well as selective associations and interpretations. Affect-congruent information is highlighted and is more likely to be accessed than any detail that is not supportive of the positive mood (Forgas 1995). 


\section{Measurement Challenges}

Self-reporting of positive affect has limitations. The inducement of positive affect through introspect or mindfulness can be particularly problematic given the difficulty of interpreting the feeling descriptions of the subjects (Isen and Erez 2007). A recommendation by Isen and Erez is to evaluate affect through multiple perspectives such as cognitive evidence, physiological measures and comparisons to a cohort that is expected to perform differently considering theoretical expectations (Isen and Erez 2007). One-item scales are considered unreliable. A preferred approach is to ask responses on a 5,7 or 9-point scale. Using synonymous terms in multiple questions can help assess the same emotional state, such as how happy, how pleased, how pleasant. When a single affective state is the focus of study, including additional affective states with multiple questions for each can improve the construct validity and allow for discriminant validation (Isen and Erez 2007).

Potential issues regarding the measurement of well being may provide insight into affect measurement challenges. Self-reported well being and health have been found to be associated in multiple studies (Croyle and Uretsky 1987; Salovey and Birnbaum 1989; Goldman, Kraemer et al. 1996; Salovey, Detweiler et al. 2001; McCaul and Mullens 2003; Barger, Burke et al. 2007; Moskowitz, Epel et al. 2008). As an example of the drawbacks in using self-reported variables, Barger and colleagues reported that negative mood was not associated with self reported health ratings when the health ratings in the 
response set were conducted with established ecological and criterion validity(Barger, Burke et al. 2007). Barger, Burke et al. highlight issues of selfreported emotional states using poorly designed survey instruments. These opposing conclusions on well-being measures highlight the challenge of emotional and affect measurement with self-reported data.

Positive affect is subject to biorhythms related to typical waking patterns in the day. Positive affect parallels the circadian rhythm of body temperature during the day. Positive affect has been measured to begin increasing one hour post waking and reach an approximate steady state approximately three hours later, peaking at the eight-hour mark. The level of positive affect slowly degrades from the eight-hour peak, experiencing an acute drop at the 12-hour post waking point. Positive affect, sleep, depression and other mood disorders all appear to be influenced by sleep patterns and energy exertion in daily cycles. Negative affect has not been observed to follow any cyclical or circadian patterns (Watson, Wiese et al. 1999). A summary of measurement methods and related considerations are provided in Table 1.

The induction of positive affect has been designed through several methods across multiple studies. The various induction methodologies are provided below in four categories for discussion, and summarized in Table 2.

The use of film clips with visual and audio stimuli has been a common and practical method for affect induction. A variation of film clips has been the use of audio only tapes. This induction method is suited to a comparison of positive, negative and neutral treatment groups and the resulting change in affect. The 
use of prerecorded induction techniques reduces variability in the treatment and potential interaction bias between the actor and study participants.

The induction of an unexpected gift has been used to observe a change in behavior or judgment between the treatment group and control. This induction method is easier to administer in a variety of settings and the time for induction effect is reduced to virtually zero. The methodology for maintaining an unexpected delivery of the gift and selecting a gift that creates the designed impact across multiple individuals must be well planned.

Word lists, meditation sessions and autobiographical recall have been used to induce affect. This intrinsic method of altered affective state can be easier to introduce and repeat periodically over time. There is potential for significant reporting bias when participants have not changed their affective state, but report such an effect.

The induction of affect can be attempted through one or more of the previously cited methods. The measurement of affect also poses challenges and threats to accuracy and reliability. The most common method of measurement is the self-reported affective state in one of many survey instruments. This allows for multiple questions to gauge constructs of interest and provides for standardized evaluation with increased reliability. A second method of measurement is the observed changes in facial expression. This observation method has the potential for inter-evaluator reliability issues. Emotional changes from happy to sad or peaceful to anxious may be better suited than milder changes in affect. The ability to observe subjects in an applied setting and 
control for environmental variables may pose challenging. A third measurement technique is to monitor behavior as the dependent variable. The induction of affect may influence behavior. The observation and measurement of behavior changes between treatment groups can provide another method of gauging the impact of affect induction.

Table 1. Positive Affect Measurement Methods

\begin{tabular}{|l|l|l|}
\hline \multicolumn{1}{|c|}{ Method of Measurement } & \multicolumn{1}{|c|}{ Advantages } & \multicolumn{1}{c|}{ Detracting Issues } \\
\hline Self Reporting Survey & $\begin{array}{l}5,7,9 \text { point scale better } \\
\text { Multiple terms improve validity }\end{array}$ & $\begin{array}{l}\text { 2 point scale unreliable } \\
\text { Single term not preferred } \\
\text { Self report difficult to validate }\end{array}$ \\
\hline $\begin{array}{l}\text { Physical Behavior, Facial } \\
\text { Expressions }\end{array}$ & $\begin{array}{l}\text { Physical evidence } \\
\text { Links emotion to physical } \\
\text { change }\end{array}$ & $\begin{array}{l}\text { Inter-observer reliability } \\
\text { May not be practical in applied } \\
\text { settings }\end{array}$ \\
\hline $\begin{array}{l}\text { Inter Group or Inter Subject } \\
\text { Comparisons }\end{array}$ & $\begin{array}{l}\text { Inter group improves } \\
\text { discriminant validity }\end{array}$ & Inter subject not as reliable \\
\hline Time of Day Considerations & $\begin{array}{l}\text { Same time measures reduce } \\
\text { biorhythm variation }\end{array}$ & $\begin{array}{l}\text { Non-standardized time } \\
\text { measurements may increase } \\
\text { variability, confounding }\end{array}$ \\
\hline $\begin{array}{l}\text { Cognitive Performance, } \\
\text { Performance of a physical or } \\
\text { mental task }\end{array}$ & $\begin{array}{l}\text { Evidence of task performance, } \\
\text { similar to physical evidence } \\
\text { Links emotion to behavioral } \\
\text { change }\end{array}$ & $\begin{array}{l}\text { May not be practical in applied } \\
\text { settings }\end{array}$ \\
\hline $\begin{array}{l}\text { Judgment, Opinion of the } \\
\text { Subject }\end{array}$ & $\begin{array}{l}\text { Allows for comparison } \\
\text { between treatment and control } \\
\text { group and differences }\end{array}$ & $\begin{array}{l}\text { Subjective judgments difficult } \\
\text { to validate }\end{array}$ \\
\hline
\end{tabular}

Table 2. Positive Affect Induction Methods

\begin{tabular}{|l|l|l|}
\hline \multicolumn{1}{|c|}{$\begin{array}{c}\text { Positive Affect } \\
\text { Induction Method }\end{array}$} & Supporting Issues for Use & Detracting Issues for Use \\
\hline Audio \& Film Clips & $\begin{array}{l}\text { Reduce variability, } \\
\text { Reduce evaluator bias }\end{array}$ & $\begin{array}{l}\text { Video not practical in applied } \\
\text { settings } \\
\text { Audio more practical }\end{array}$ \\
\hline Unexpected Gifts & Suited for applied settings & $\begin{array}{l}\text { Repeated induction may reduce } \\
\text { effect }\end{array}$ \\
\hline $\begin{array}{l}\text { Autobiographical } \\
\text { Recall }\end{array}$ & $\begin{array}{l}\text { Tailored for each individual } \\
\text { Suited for applied settings }\end{array}$ & Difficult to appraise true impact \\
\hline Word Lists & $\begin{array}{l}\text { Reduce variability } \\
\text { Reduce evaluator bias } \\
\text { Can be tailored to individuals }\end{array}$ & Difficult to appraise true impact \\
\hline
\end{tabular}




\section{Positive Affect Impact}

The influence of positive affect on select thought processes is subtle. Individuals may not notice the effects of positive feelings, but it has been documented that the impact of even small changes in positive affect can influence decision-making, leading to more efficient and productive decisions and solutions to problems (Isen 2008). The positive yet small events we run across in our daily living are adequate to induce this positive affect (Isen 1999). As an example, individuals who viewed a film intended to induce a positive mood rated unfamiliar words more positively than subjects in a neutral film comparison group. Simple daily events that can induce positive affect, even mildly, impact behavior and the openness to new ideas (Isen, Daubman et al. 1987). Individuals finding a dime in a public telephone (1978) were more likely to help a stranger pick up dropped papers, compared to those who did not find a dime (Isen, Shalker et al. 1978).

Affect can have several functions during the information-processing phase of decision-making, highlighting specific information. Positive affect opens up channels for new information to be considered. This new information is then fed into the evaluation of information, as well as providing an influence on the motivation, if action is part of the final decision (Diffenbach, Miller et al. 2008). Lyubomirsky and colleagues examined studies that included over 275,000 participants on the topic of happiness, positive affect and well-being. Individuals who express happiness, which could be interpreted as positive emotions, have more success in life in the domains of work ( $r=.17)$, relationships $(r=.17)$ and 
health ( $r=.32)$ (Lyubomirsky, King et al. 2005). This meta-analysis concludes that happiness precedes favorable life-events such as hiring, productive work and fulfilling efforts across multiple studies with consistent results. The key point in this conclusion is the temporal relationship of happiness preceding success (Lyubomirsky, King et al. 2005; Pressman and Cohen 2005).

Positive affect influences the recall of memories and cognitive processing and can be considered to serve as information (Forgas 1995), as well as providing directional influence on decisions (Diffenbach, Miller et al. 2008). Participants in a positive affect state concluded they obtained all necessary information in a reading exercise in less time compared to the negative affect group (Martin, Ward et al. 1993). Smith and Shaffer conducted several experiments to explore the influence of positive affect on cognitive processing. The evidence appeared to support parallel processing of emotion states and messages. Emotions and affect influence cognitive processes in separate pathways rather than over the same circuit and operate as their own separate process. The impact of positive affect did not appear to decrease cognitive capacity and increased motivational interpretation (Smith and Shaffer 1991).

Positive affect has been shown to stimulate an increase in elaboration when the topic context is neutral or positive. When an individual is performing a task, or is ready to start a given task that is enjoyable, the induction of positive affect will result in increased effort or an elaboration increase. If the same positive affect induction occurs when the individual is performing a distasteful task, the elaboration and increased effort does not take place. A negative task or 
tedious material is not influenced when positive affect is induced. The increase in elaboration for the positive and neutral material draws upon a broader set of cognitive information and increases the creative or flexible options for consideration (Isen 2008). Positive affect may not have much of an impact on motivation. Isen and Reeve noted that motivation as measured by desire to complete a dull, routine work task was not effected by positive affect (Isen and Reeve 2005). This same research question was studied; researchers did observe an increased liking when the task was considered enjoyable (Kahn and Isen 1993). The resulting influence of the positive affect inducement is selective, based on the individual's competing considerations such as goals, barriers, fears, and competing tasks. The positive affective state is not a catalyst for all action, but a selective influence based upon any one of multiple variables (Isen 2008). Positive affect appears to cue the memories of positive experience and serves to influence the cognitive process. The influence of positive affect becomes selective, that is negative emotions or issues viewed as negative are not reversed. Positive affect influence is observed to impact positive issues primarily (Isen, Shalker et al. 1978; Teasdale and Fogarty 1979; Teasdale and Russell 1983). Positive affect influences individuals to favor self-protection and the maintenance of the status quo; it is risk-averse (Isen 2000).

Positive affect has been observed to influence decision making for at least four hours (Isen and Levin 1972; Isen, Daubman et al. 1987) after induction. When daily inductions were affirmations and word list recitals, over a multi-week period the positive affect scores were maintained for several days after 
withdrawal of the inductions (Fredrickson and Branigan 2005). Positive affect has been measured in a variety of settings including college students, employer worksites, physician practice offices, consumer retail locations and business negotiation context (Isen and Levin 1972; Carnevale and Isen 1986; Isen, Daubman et al. 1987; Hakn and Isen 1993; Kahn and Isen 1993; Estrada, Isen et al. 1994; Estrada, Isen et al. 1997; Ashby, Isen et al. 1999; Hermalin and Isen 2008).

There are several related cognitive skills that are influenced by the positive affect state. Research provides evidence that positive affect enhances problem solving, creativity, innovation, social interaction such as helpfulness, friendliness, socially responsible behavior, and motivation for a variety of tasks (Isen and Levin 1972; Hakn and Isen 1993; Estrada, Isen et al. 1994; Bless, Clore et al. 1996; Salovey, Detweiler et al. 2001; Erez and Isen 2002; Dreisbach and Goschke 2004; Fredrickson and Branigan 2005; Chapman and Coups 2006; Isen 2008). Decisions reached under a state of positive affect can be expected to draw upon more creative, flexible and wider set of considerations than decisions not influenced by positive affect (Estrada, Isen et al. 1994; Isen 1999). This selective extraction of positive memory material accounts for the specificity of positive affect. Positive affect draws upon previously stored memories to stimulate new cognition, rather than a blanket influence that impacts all cognitive functions. Individuals who are more positive feel less threatened compared to individuals who feel more negative and behave cautiously for current tasks, and are more open to debate logic (Bless, Bohner et al. 1990). 
Humor research may have applications for positive affect induction. Adaptive humor styles include facilitative, affiliative, and self-enhancing humor. The use of humor under adverse conditions increases the coping skills of individuals with these traits (Kuiper and McHale 2009). The use of humor in a facilitative fashion leads to higher levels of social self-esteem and lower levels of depression. Individuals compare their personal situation to others in their social circles or networks. The self-evaluation of social standing includes an interaction effect with emotional and affective state (Aspinwall and Taylor 1993). Study participants rating the disposition of individuals under positive affect provide higher, more positive appraisals (Forgas and Bower 1987). Participants in a positive affective state are more likely to make value decisions that are congruent with implicit values compared to participants in negative affect. As an example, when an individual is in a positive emotional state the world is perceived to be more positive. Individuals who are more negative in emotional state have a tendency to see the world in a more negative perspective. Thus, participants in positive emotional state rely on previously stored positive values to render decisions (Huntsinger and Smith 2009).

Positive affect influences motivation and task performance. Study participants in the positive affect treatment group performed better, were more persistent in task activity, approached assigned tasks with increased intensity and reported higher motivation levels compared to participants receiving neutral mood treatments (Erez and Isen 2002). When an individual considers tasks to be negative, unsolvable, unpleasant or distasteful, the influence of positive affect 
has not been observed (Isen, Daubman et al. 1987; Kahn and Isen 1993; Isen and Reeve 2005). There is impact on both intrinsic and extrinsic expectancy considerations when positive affect is present (Isen and Reeve 2005). Positive affect can be viewed as one component in the decision-making process. This process recognizes multiple variables that interact and influence the final outcome or decision. The variables involved in decision-making include the task motivation or desire to complete, the priority status of the task, the positive or negative context of the task environment, individual motivation and personal goals surrounding the context of the task. The influence of positive affect on cognition is selective. The decision-making process or cognitive effort for negative topics or disliked options is not impacted by a positive affect state. Positive affect has been observed to impact decision-making and other cognitive functions, only when the topic or material is of a neutral or positive. The final degree of influence or positive affect impact is determined at the intersection of several factors. The context or milieu of factors that determine the degree of positive affect influence includes the novelty or desire to engage in the target behavior, the utility of the decision, the general context and how the behavior is viewed, the degree that the target behavior compliments other goals and motivations. These factors interact and provide the general context to guide the total impact of positive affect (Isen 2008). Individuals in a happy or positive affect condition make use of heuristics and common patterns to interpret problems and information (Bless, Clore et al. 1996). 
The influence of positive affect is stronger when the individual is not in a negative state and when the strength of the affective induction is moderate. The following studies by Kahn and Isen; Isen, Daubman et al; and Roehm and Roehm are informative regarding these patterns. When study participants were provided a choice of new food products, the positive affect influence was only present when the choice was considered favorable. Participants were willing to consider new products if those products were viewed in a positive context. Products that were thought to taste bad or were totally unfamiliar were not selected (Kahn and Isen 1993). When study participants rated unfamiliar words after positive affect induction viewing a film clip, ratings were higher than neutral treatment participants (Isen, Daubman et al. 1987). Mild positive affect increased variety-seeking behavior for new food products. In mild positive affect groups; likelihood to seek variety was higher than both neutral and extreme positive mood. There appears to be a curvilinear or inverted-U response to positive affect dose for cognitive effect reported in multiple studies; affect induction by video clip on product decisions (Roehm and Roehm 2005); emotional levels on food choices (Macht 2008); affect induction on product choices (Kahn and Isen 1993).

A study of decision-making by physicians after positive affect induction displayed the positive affect impact. Physicians in the treatment group reached a diagnosis decision faster and were willing to consider alternate diagnosis to a greater degree compared to neutral affect physicians. This influence on decision making highlights the increased creativity and problem solving cognitions that positive affect can create (Estrada, Isen et al. 1997). 
Positive affect was observed to influence negotiation behavior.

Negotiation study participants reported their emotional state as more positive and more pleasant than control participants. Qualitative assessment of confrontational negotiation techniques in positive affect participants was less than the control group (Carnevale and Isen 1986).

Negotiators who were placed in a position of control displayed an interaction effect with emotion, happy vs. angry. Participants in a happy emotional state were more likely to accept a disadvantaged financial offer (Andrade and Ariely 2009).

A summary of reviewed literature studies is provided in Table 3. The study, lead author, induction method and dependent variables including positive affect change are summarized. 
Table 3.

Summary of Positive Affect Studies

\begin{tabular}{|c|c|c|c|c|c|}
\hline & & & Table 3. & & \\
\hline & & & & & \\
\hline $\begin{array}{l}\text { Lead } \\
\text { Study } \\
\text { Author }\end{array}$ & $\mathbf{n}$ & $\begin{array}{l}\text { Induction } \\
\text { Technique }\end{array}$ & $\begin{array}{l}\text { Emotion/Affect } \\
\text { Results }\end{array}$ & Task & Dependent Measure Results \\
\hline & & & & & \\
\hline Andrade & 110 & Film Clips & $\begin{array}{l}F(1,49)=40.9 \\
p<.001, n 2 p=.45\end{array}$ & Negotiation & $\begin{array}{l}M(\text { happy })=\$ 8.0, S E=.39 ; F(1,49) \\
=3.15, p=.08, n 2 p=.06\end{array}$ \\
\hline Aspinwall & 80 & $\begin{array}{l}\text { Autobiography } \\
\text { recall }\end{array}$ & $\begin{array}{l}M s=40.33 \text { vs. } 22.2 \\
F(1,76)=135.46 \\
p<.0001\end{array}$ & $\begin{array}{l}\text { Social } \\
\text { Comparison }\end{array}$ & $F(1,77)=20.65, p<.0001$ \\
\hline Barger & 168 & Film Clips & $\begin{array}{l}M=13.89 \text { vs. } 3.86 \\
t(165)=-12.06 \\
p<.001\end{array}$ & $\begin{array}{l}\text { Self Reported } \\
\text { Health }\end{array}$ & $\begin{array}{l}t(164)=0.51, p=.61 \text { [evidence not } \\
\text { found] }\end{array}$ \\
\hline Bless & 82 & $\begin{array}{l}\text { Autobiography } \\
\text { recall }\end{array}$ & $\begin{array}{l}M=7.1 \text { vs. } \\
5.4, F(1,78)=16.19 \\
p<.001\end{array}$ & $\begin{array}{l}\text { Use of memory } \\
\text { heuristics }\end{array}$ & $\begin{array}{l}M s=67.0 \% \text { vs. } 57.9 \% \\
F(1,78)=5.64, p<.02\end{array}$ \\
\hline Bless & 87 & $\begin{array}{l}\text { Autobiography } \\
\text { recall }\end{array}$ & $\begin{array}{l}M=7.0 \text { vs. } 6.1 \\
F(1,70)=5.01 \\
p<.03\end{array}$ & $\begin{array}{l}\text { Attitudes towards } \\
\text { an argument }\end{array}$ & $F(2,58)=5.65, p<.01$ \\
\hline Bless & 75 & $\begin{array}{l}\text { Autobiography } \\
\text { recall }\end{array}$ & $\begin{array}{l}M=6.3 \text { vs. } 5.4 \\
F(1,67)=4.18 \\
p<.04\end{array}$ & $\begin{array}{l}\text { Attitude under } \\
\text { positive mood } \\
\text { and distracted }\end{array}$ & $\begin{array}{l}F(2,61)=3.02, p<.06 \text {, [evidence not } \\
\text { found] }\end{array}$ \\
\hline Carnevale & 80 & Cartoons + gift & $F(1,36)=5.59, p<.03$ & Negotiation & $F(1,26)=16.9, p<.01$ \\
\hline Croyie & 44 & Film Clips & $\begin{array}{l}M=7.67 \text { vs. } 3.48 \\
t(42)=9.29, p<.001\end{array}$ & $\begin{array}{l}\text { Self Reported } \\
\text { Health }\end{array}$ & $M=7.67$ vs. $6.83, t(42)-2.07, p<.05$ \\
\hline Dovidio & 132 & Candy gift & $\begin{array}{l}F(1,81)=7.24 \\
p<.014\end{array}$ & Group Appraisal & $M s=4.59$ vs 3.58 \\
\hline Isen & 65 & Film Clips & $t(25)=2.00, p<.05$ & $\begin{array}{l}\text { Candle task } \\
\text { puzzle }\end{array}$ & $\times 2(1, N=27)=8.19, p<.01$ \\
\hline Erez & 97 & Candy gift & $\begin{array}{l}\mathrm{M}=3.83 \text { vs } 3.57 \\
\mathrm{~F}(1,96)=4.05, \mathrm{p}<.05\end{array}$ & Solve anagrams & $\begin{array}{l}M=3.6 \text { vs. } 2.77, F(2,48)=4.36 \\
p<.05\end{array}$ \\
\hline Estrada & 44 & Candy gift & not tested & creativity test & $47 \%$ vs. $13 \%, p=0.028,1$ tailed \\
\hline Estrada & 44 & Candy gift & not tested & clinical case study & Time $20 \%$ vs $39 \%, t=2.85, p=.008$ \\
\hline Forgas & 52 & $\begin{array}{l}\text { Feedback on a } \\
\text { bogus test }\end{array}$ & $\begin{array}{l}F(1,50)=6.54 \\
p<.025\end{array}$ & \begin{tabular}{|l|}
$\begin{array}{l}\text { Appraisal of } \\
\text { individuals }\end{array}$ \\
\end{tabular} & $\begin{array}{l}3.87 \text { vs, } 6.19, F(1,50)=14.25 \\
p<.01\end{array}$ \\
\hline Futterman & 66 & $\begin{array}{l}\text { Actors using } \\
\text { method acting } \\
\text { technique }\end{array}$ & $M=5.51$, cut - off 4.0 & $\begin{array}{l}\text { Immune } \\
\text { Responses }\end{array}$ & $C D 8$ counts $F(1,16)=5.16, p<.04$ \\
\hline
\end{tabular}




\begin{tabular}{|c|c|c|c|c|c|}
\hline & & & Table 3. & & \\
\hline & & & (Continued) & & \\
\hline $\begin{array}{l}\text { Lead } \\
\text { Study } \\
\text { Author }\end{array}$ & $\mathbf{n}$ & $\begin{array}{l}\text { Induction } \\
\text { Technique }\end{array}$ & $\begin{array}{l}\text { Emotion/Affect } \\
\text { Results }\end{array}$ & Task & Dependent Measure Results \\
\hline & & & & & \\
\hline Isen & 66 & Candy gift & not tested & $\begin{array}{l}\text { Motivation } \\
\text { appraisal using } \\
\text { Puzzles }\end{array}$ & $\begin{array}{l}M=233.3 \text { vs. } 127.4 \\
5(58)=2.35, p<.05\end{array}$ \\
\hline Isen & 52 & Cookies & not tested & Willing to help & $t=1.96, p<.05$ \\
\hline Isen & 74 & $\begin{array}{l}\$ 0.10 \text { in } \\
\text { telephone }\end{array}$ & not tested & Willing to help & $F(1,50)=8.22, p=0.006$ \\
\hline Macht & 210 & $\begin{array}{l}\text { Self-induced } \\
\text { emotion with } \\
\text { word list }\end{array}$ & not tested & $\begin{array}{l}\text { Self Reported } \\
\text { eating behavior }\end{array}$ & $\begin{array}{l}\text { Anger/Joy vs, fear/sadness - higher } \\
F(557,3)=24.95, p<0.001\end{array}$ \\
\hline Martin & 51 & Film Clips & $\begin{array}{l}M=3.91 \text { vs } 2.64 \\
F(1,47)=49.23 \\
p<.001\end{array}$ & $\begin{array}{l}\text { Time to reach } \\
\text { cognitive goal }\end{array}$ & $\begin{array}{l}191.7 \mathrm{~s} \text { vs. } 149.63 \mathrm{~s}, \mathrm{~F}(1,47)=9.46 \\
p<.003\end{array}$ \\
\hline Roehm & 55 & Film Clips & $\begin{array}{l}M=4.89 \text { vs. } 3.75 \\
F(2,29)=25.79 \\
p<.01, n 2=.50\end{array}$ & $\begin{array}{l}\text { Variety seeking in } \\
\text { products }\end{array}$ & $F(2,57)=11.38, p<.01, n 2=.21$ \\
\hline Salovey & 66 & Audio Clip & $\begin{array}{l}\text { Wilks's }=0.36 \\
F(4,120)=20.02 \\
p<.0001\end{array}$ & $\begin{array}{l}\text { Reported physical } \\
\text { symptoms }\end{array}$ & $F(1,63)+8.38, p<.005$ \\
\hline Smith & & Film Clip & $\begin{array}{l}M=28.54 \text { vs } 23.56 \\
F(1,53)=4.55 \\
p<.05\end{array}$ & $\begin{array}{l}\text { Attitudes towards } \\
\text { a target issue }\end{array}$ & $\begin{array}{l}M=5.17,6.31 \text { vs. } 5.23,6.79 \\
\text { [evidence not found] }\end{array}$ \\
\hline Teasdale & 32 & $\begin{array}{l}\text { Self-induced } \\
\text { emotion with } \\
\text { word list } \\
\end{array}$ & $\begin{array}{l}66.7 \text { vs } 31.8 \\
t(11.78), p<0.001\end{array}$ & Word recall & $F(2,62)=6.27, p<0.01$ \\
\hline
\end{tabular}

\section{Health Impact of Positive Affect}

The influence of positive affect on any health related decision is the arena that explores the translational research aspect of the many previous findings. The theory of self-regulation outlines a constantly fluid set of decision points for an individual to navigate (Diffenbach, Miller et al. 2008). These decisions are both deliberate and without forethought but more reflexive. Individuals in a positive affective state self report increased well-being compared to negative affective state individuals who report negative judgments of health (Croyle and Uretsky 1987; Ryan and Frederick 1997). An association between self-rated health and positive affect in blue-collar women workers was observed, even after controlling for age, education, BMI, negative affect and stress (Kelsey, DeVellis 
et al. 2006). Isen proposes that interventions that influence affect or emotion may be as effective as direct behavioral interventions (Isen 2001). Andrade and Ariely conclude that decisions based on a fleeting affect can influence not only a proximate decision but also further decision-making processes that are more distal (Andrade and Ariely 2009). The literature is informative regarding the influence of emotions and affect on a variety of decisions. There is no evidence that supports excluding health behavior decisions. The inclusion of emotion or affect as a component of behavior change interventions is suggested as a further research by Isen, Andrade and Ariely and Macht (Isen 2001; Macht 2008; Andrade and Ariely 2009).

The use of positive affect is not a panacea for all health behaviors. Positive affect will not reverse a divergent disposition towards a behavior. The greater the divergence between the current and desired behavior the less the influence of a positive affect induction (Lawton, Conner et al. 2009). Positive emotion induction is suggestive of a yield for a positive influence on long-term health. The influence of positive affect across multiple organ systems such as heart rate, respiration rate and endocrine secretion process supports the broad based influence and the prospective contribution of positive emotion as a limiting effect on disease development (Richman, Kubzansky et al. 2005).

A study by Zinser et al. found that urges to smoke for individuals attempting to quit were negatively associated with positive affect (Zinser et al in (Lyubomirsky, King et al. 2005)). When positive affective attitude was at odds with a health behavior, the influence of the positive affect diminished (Lawton, 
Conner et al. 2009). Lawton et al. regressed affective attitude on preventive health behaviors. The health promoting behaviors of exercise, fruit and vegetable intake and vitamin supplement intake were not significant, even in the presence of strong affective attitude and intention relating to these behaviors. Behaviors that are enjoyable such as tobacco use or alcohol intake, but viewed as harmful are more likely to display low levels of successful change, even when intention values are high. Lawton et al. suggest that affect and emotions are strong factors in health behavior decisions and may even exceed cognition in self-reported intention (Lawton, Conner et al. 2009). The literature review performed by Lawton et al. examined 225 scientific papers. Cross-sectional, longitudinal, and experimental data supported the conclusion that happy disposition or positive affect preceded the higher health status and increased adherence to healthier behaviors.

The broaden and build theory outlined how positive affect can both influence immediate decision-making and cognitive processes as well as build a repertoire of positive affect memories for future use (Fredrickson and Branigan 2005). The use of a health behavior such as physical activity may present the opportunity to be an additive influence in the increase of positive affective states (Lyubomirsky, King et al. 2005). The positive affect emotion is positively correlated with higher levels of physical exercise. This outcome can lead to increased health benefits through the indirect inducement of positive affect (Lyubomirsky, King et al. 2005). Runners reported a higher positive emotional level compared to anaerobic exercisers. The mechanism that drives the 
increased positive affect has not been proven, but aerobic exercise is suggestive of creating positive affect (Dyer and Crouch 1988). When positive emotions are detailed with specific emotional types, joy has been associated with increased physical play and exploration that is suggestive of an arousal component (Fredrickson 1998). The induction of positive affect and subsequent engagement in exercise may initiate a cycle of broaden and build processes similar to those outlined by Fredrickson (Fredrickson 2001).

Mood has been correlated to self-efficacy and is a predictor of health behaviors such as a healthy diet or smoking cessation. Individuals who are happier rate their self-efficacy higher when considering health-promoting behaviors. Salovey and Birnhaum conducted three separate experiments to evaluate the influence of emotion on symptom appraisal, self-efficacy, outcome expectations and vulnerability perceptions. Happy, sad and neutral affect inductions were introduced to subjects ( $n=66$ undergraduates) with acute illness symptoms of cold or flu, in two studies and healthy state subjects in the third study. The affect inductions were autobiographical recalls administered through an audiotape. Individuals in a happy state were more likely to indicate an intention to make health-promoting decisions regarding cold and flu treatment and protective behaviors in the future and were somewhat more likely to believe these actions would improve health status. Individuals in a sad state were more likely to report more ill symptoms and less confident they could implement these health-promoting behaviors (Salovey and Birnbaum 1989). 
The consumption of food can induce pleasure through satiety and when alleviating the negative effects of hunger and energy deficit (Macdiarmid and Hetherington 1995). Negative emotions have been associated with food craving in study participants (Hill, Weaver et al. 1991). When carbohydrates are consumed the amino acid tryptophan becomes more available and subsequently increased serotonin levels (Fernstron \& Wurtman 1971 in (Macdiarmid and Hetherington 1995)). Interestingly, negative moods associated with craving chocolate were not relieved after consumption. Weingarten \& Elston found that the consumption of chocolate to satisfy a chocolate craving resulted in a negative affect in women compared to men (Weingarten \& Elston 1991 in (Macdiarmid and Hetherington 1995). These findings support the subjective expected pleasure theory of Mellers and McGraw, that individuals evaluate anticipated pleasure, and then relate this to stored information, and use this memory to value the current decision option. Related to this anticipatory phase, individuals who failed in dieting behavior were less displeased than they had predicted (Mellers and McGraw 2001).

Mood may impact health behavior by mediating self-efficacy for health related decisions. The arousal of emotions may result in judgment shifts and the cueing of congruent memories as part of the health related decision-making process. These differences in the motivating properties of affective arousal have been termed emotional intelligence (Salovey and Birnbaum 1989). Our decision making process appears to be a confluence of both cognitive and emotional factors. Our daily decisions on health related issues are driven to at least some 
degree by our feelings (Watson, Wiese et al. 1999; Lawton, Conner et al. 2009). The clustered relationship between positive affect, sleep and mood disorders reflect energy expense and conservation as a continuous cycle (Watson, Wiese et al. 1999).

A 5-way model has been developed by Macht to explain the impact of emotions on eating behavior (Macht 2008). Three dimensions of emotions are integral to the eating behavior model. The emotional dimensions in Macht's model include valence, arousal and intensity. Emotions that are cued by food can affect food selections. Strong or extreme emotions will suppress eating as a result of emotional responses that are not congruent with food intake. Reported hunger was more intense during negative emotions. Anger, sadness and fear seem to influence binge eating. Joy increases hedonic consumption (Macht 1999; Macht and Simons 2000; Macht 2008). Moderate emotions influence eating behavior dependent on the motivation at the time to eat. An individual, who wants to restrain eating, increases food intake with negative and positive emotions due to the impairment of cognitive control through the emotional inducement. The sensation of hunger and other stomach sensations were observed among respondents in negative affective states, but not positive states. This cross-cultural study included 779 respondents in eight European countries. Anger, sadness and fear elicited these stomach sensations but joy did not (Macht and Simons 2000). Food intake that is driven by an emotional basis results in increased high-fat and sweet foods. An individual who is in a normal eating state is influenced by emotions in congruence with their cognitive and motivational 
features (Macht 1999; Macht and Simons 2000; Macht 2008). Although Macht's model is specific to eating behavior, the conclusions in research studies are supportive of other findings regarding the influence of positive affect on health behavior. Extremes of low or high emotional induction can lead to adverse decisions regarding a target behavior. Moderate emotional inductions contribute to more open-minded decision-making (Isen, Daubman et al. 1987; Salovey and Birnbaum 1989; Isen 2001; Isen 2008; Macht 2008). Positive affect induces individuals to make healthier eating choices, as long as the individual is not in a negative emotional state (Macht 2008).

The five-way model is illustrated in Figure 3 . The five antecedents to eating behavior are depicted on the left. Each situation leads to a subsequent influence on eating behavior. The intake of food is a stimulus that impacts food selection. Extreme emotion tends to suppress food intake. Moderate emotional arousal has the greatest influence on food decisions. Individuals who are restraining their eating intake exhibit a loss of control. Individuals who are in an emotional eating state increase intake when a negative affective state is reached or induced. 
Figure 3. (Macht 2008)

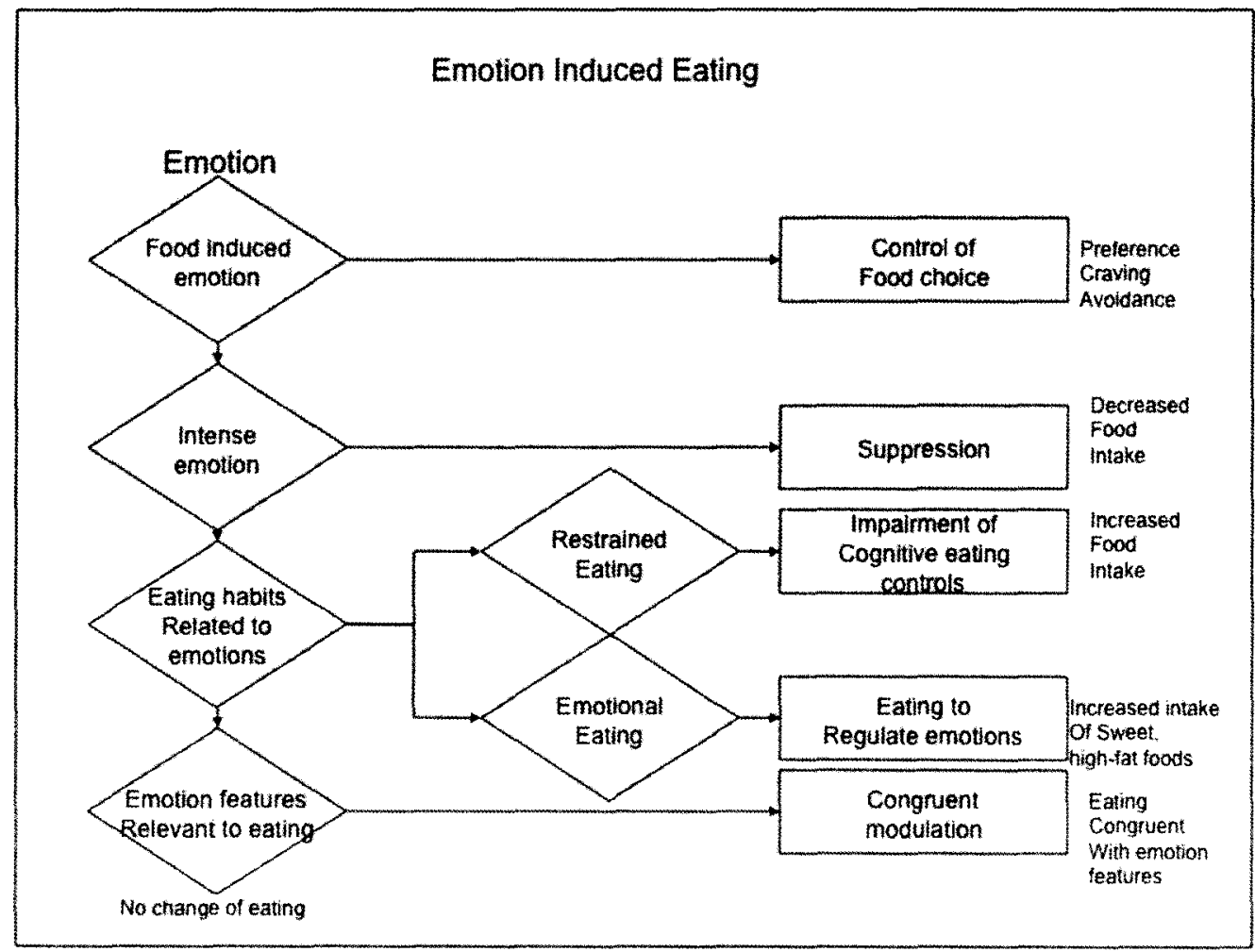

\section{Conclusion}

This review outlined the similarities and differences between mood, emotion and effect. The transitory emotion and affect have been shown through the multiple studies cited to influence the decision making process. Specifically, the focus of these studies was on positive affect. The influence of positive affect on cognitive processes was evidenced in diverse tasks such as product evaluations; puzzle completions, word associations, and personality appraisals. The extreme ranges of positive affect valence were not as effective as moderate affect on improving performance in these tasks. Light to moderate inducement of positive affect has been shown across this range of tasks to improve the decision-making process and task outcomes. These findings can be considered 
for use to compliment current behavioral change interventions. The potential implications are an increased effectiveness of behavioral changes using positive affect.

The specificity of influence observed and the cueing effect on positive material can yield an upward spiral of positive emotions. This selective impact requires targeted use of positive affect, since the result of positive affect inducement is not a consistent positive bias across all cognitive material. The treatment of positive affect as information to be accessed in a cognitive process, allows for straightforward intervention designs for health issues. The study of cerebral processes involving cognition and emotion lend additional insight to the physical explanation for causal factors of positive affect. The evidence that supports the influence of dopamine on activation patterns and neural firings supports the conclusion that positive emotions are treated as information within cognitive decision-making processes.

The traits associated with an extrovert, including positivity may be supportive of an association between improved health and optimism. This approach to health through positive emotions is a relatively recent field of study compared to the studies around the therapeutic value of cognitive behavioral therapy. The experiential research cited in this paper concludes that positive affect improves creativity, problem solving, innovation, social interaction and task motivation. The implication of incorporating this influence into health behavior interventions has tremendous potential. Recent studies have begun to explore this aspect of positive affect influence on health decisions. Similar to the 
processes associated with the stages of change theory (Prochaska, Velicer et al. 1994), or the influence of self-efficacy on behaviors (Bandura 2001), the use of positive affect may merit consideration as a component in health behavior change models.

\section{Positive Affect Induction}

There are various types of positive affect induction that include imagery, audiotapes, humorous videotapes, video clips, reflection on positive scenarios, autobiographical recall of positive events, fragrance, personal diary of positive events, candy, positive word list/association (Lyubomirsky, King et al. 2005; Isen and Erez 2007). The most important factor in consideration of introducing positive affect, is the amount of time or latency, an individual is influenced rather than the intensity of the affect (Lyubomirsky, King et al. 2005). Studies previously cited are presented in Table 3 and constitute controlled laboratory studies that have not been tested in an applied setting.

The evaluation of positive affect inductions in applied settings across multiple days may be influenced by factors not previously identified. As an example, there is a definite circadian rhythm to levels of positive affect. After rising from sleep, positive affect increases and maintains levels throughout the day, peaks at approximately 8-hours post waking, and then decreases in the evening (Watson, Wiese et al. 1999; Lawton, Conner et al. 2009).

The use of autobiographical recall or similar intrinsic induction methods have been criticized for the lack of validation and inability to eliminate a reporting bias (Isen and Erez 2007). The use of video clips has been reported in several 
studies with reports of successful affect induction (Croyle and Uretsky 1987; Isen, Daubman et al. 1987; Smith and Shaffer 1991; Martin, Ward et al. 1993; Roehm and Roehm 2005; Barger, Burke et al. 2007; Andrade and Ariely 2009). Audio tape induction methods have the potential for delivery via telephone or digital recordings and have been reported successful (Salovey, Detweiler et al. 2001). Word lists have been induced in printed or audio media and reported successful (Teasdale and Fogarty 1979; Teasdale and Russell 1983; Macht 2008).

\section{Research Need and Problems}

The research implications and opportunities to explore the impact of positive affect on health care decisions and behavior appears to be numerous. Meller's and McGraw's subjective expected pleasure theory has not been applied to health and medical decision making situations (Mellers and McGraw 2001). The role of emotions has not been applied to personal decisions regarding medical care and preventive care (Diffenbach, Miller et al. 2008), and is recommended for additional study of nutritional intake (Macht and Simons 2000). It appears that depressed affect supports indecisiveness, yet the impact of this circumstance on health related decisions has not been directly explored (Consedine 2008). The effects of specific emotions such as anger, sadness and fear has not been reported (Macht 2008). It appears that evidence is absent regarding the effect of positive affect on coping behavior and health behaviors (Lyubomirsky, King et al. 2005). 
The literature reviewed appears to be missing several facets of positive affect study. Practical application of induction techniques outside of controlled conditions has not been reported. The use of the telephone has been reported for lifestyle behavior change interventions (Wing, Jeffery et al. 1996; Bickmore and Giorgino 2006; Eakin, Lawler et al. 2007; Busey and Michael 2008; Estabrooks and Smith-Ray 2008; Newman, Flatt et al. 2008; Tucker, Cook et al. 2008; Estabrooks, Shoup et al. 2009), but a phone-based positive affect induction intervention was not found in the literature. A multi-week correlation study of self-reported emotions has been published (Macht and Simons 2000), but no prospective randomized experimental study of positive affect was found. 


\section{CHAPTER III \\ METHODS AND MATERIALS}

\section{The Research Problem}

Can positive affect be induced in an applied setting across multiple days, comparing treatment and control groups?

A secondary research question is, will the induction of positive affect through a telephone based interactive voice response system (IVR) increase positive affect in the treatment group compared to a control group? The instrument to assess this change is the PANAS.

Another secondary research question framed becomes, will the induction of daily positive affect be an effective means of driving an increase in daily physical activity in patients in the treatment group compared to a control group in a community environment.

Exploration of additional research questions include, will the induction of daily positive affect influence behavioral change factors, including self-efficacy, intention, motivation, and cognitions regarding physical activity.

Physical activity will be monitored through a memory chip enabled pedometer for daily activity. A self-report questionnaire for physical activity will also be collected. Additional parameters to substantiate behavioral change include the following measures; daily call acceptance, phone call completion rate. 


\section{Research Design and Methods.}

This study is designed as a practical exploratory clinical trial in that it will:

- Compare clinically relevant alternative treatment interventions in an applied setting;

- Recruit participants from heterogeneous family practice medical settings through the University of Louisville medical offices;

This limited study is a short-term project that is designed to evaluate the ability to induce positive affect through phone contact or other methods on a daily basis. This study will provide results that will deliver valuable knowledge for use in a larger clinical trial to be conducted later, which may evaluate longer-term impact of positive affect on health behaviors. A pilot study will be conducted prior to the primary study start and is described later. The pilot study will test induction messages and serve to test protocols and study designs.

The evaluation of positive affect influence through daily interactions Monday - Saturday, as a method of behavioral change is the primary research objective. The evaluation period will be two weeks, a two-week run-in and final outcome measurements one week after the final positive affect induction. The total timeframe is five weeks from screening session to the final outcome measure.

A two-arm crossover study is designed with a third usual care arm. The study arms are:

- No daily treatments,

- Daily treatments for positive affect induction 
- Daily treatments with no positive affect induction

\section{Study Participants}

The approval of human subject research was sought through the University of Louisville Institutional Review Board. Approval was granted as case 10.0219 dated June 18, 2010. An initial pool of 20,000 patients that includes 9,700 female patients is the expected initial sample frame requirement. Medical provider offices in family medicine will be solicited to assist in the recruitment of study participants. Eight to ten family practice medical providers, with a patient panel of 3,000 to 5,000 individuals per provider in the University of Louisville Physician Practice will be recruited to pre-screen and obtain volunteers for the study. Orientation sessions for physicians and office staff will be conducted to stimulate the recruitment effort. Posters and information flyers will be provided for the medical practice staff and candidate participants. Medical offices in locations of high-density ethnic diversity within urban Louisville, Kentucky will be

sought. A cohort tree diagram in Figure 4 illustrates the flow and branching from the initial pool of 20,000 total, with 9,700 females into the eventual two treatment arms and one control arm of the study.

Each medical provider is expected to examine 300 female patients within a one-month recruitment window. 3,000 patients from the 8-10 physicians are expected to be potential study volunteers. A single page recruitment memo in a sealed envelope will be distributed to each potential study participant, who meet the inclusion criteria and volunteer during a routine outpatient office. When available, female patients who meet the BMI criteria $\left(27 \mathrm{~kg} / \mathrm{m}^{2}\right.$ to $\left.35 \mathrm{~kg} / \mathrm{m}^{2}\right)$, will 
be contacted through a recruitment letter from the treating medical provider. The memo will provide an overview of the proposed study and be signed by the study investigator and medical provider. The 3,000 physician office contacts and 7,000 postal letters, for a total of 10,000 recruiting contacts are the projected pool required.

Although cross sectional and correlation analysis reveal similar selfreported emotion and mood scores between gender (Watson 2005), emotional reactions and transitional states relative to preventive health care and weight management behaviors are significantly different between women and men (Wardle, Haase et al. 2004), and may be attributed to both physiological and socio-cultural factors (Huntsinger and Smith 2009). Although the research intent of this study is limited to the subject of positive affect and physical activity, future investigation may include the study of various health behaviors. The screening and selection of participants is designed to reduce potential variations and generally include females who are likely to initiate a physical activity or weight management program in the future. The upper limitation of BMI within the sampling frame is designed to reduce potential confounding emotional and psychological variables. Psychological dysfunction is associated with the severe obese to a greater degree than overweight or level 1 obesity (Friedman and Brownell 1995; Ball and Crawford 2006; Maio, Haddock et al. 2007).

\section{Participant Screening}

$10 \%$ of the initial 9,700 females $(n=970)$ are projected to volunteer or express an interest in participating. $30 \%$ of the volunteers $(n=290)$ are expected 
to meet the BMI inclusion criteria. Individuals who express an interest in study participation will be asked to return an interest form and review of general study information to the study investigator via postal mail, electronic mail or by phone. Communication type will be the preference of each individual. Prospective participants will be recruited to complete an intake questionnaire with which to determine preliminary eligibility. An informed consent form will also be forwarded for review prior to the initial interview.

\section{Pilot and Validation Study Methodology}

A group of 50 participants will be segregated from the cohort of 290 after preliminary eligibility is determined and prior to randomization into the treatment and control arms (Figure 4). The 50 volunteers in the validation study are projected to yield 30 participants, a $40 \%$ erosion due to filtering from inclusion and exclusion criteria, to begin this pilot phase. The pilot study is intended to increase the accuracy of the induction methodology and serve as a validation of the screening procedures and other methods. Participant tolerance for the daily phone calls and pedometer use will also be validated.

A pilot study is planned to validate positive affect induction methods. A qualitative study of induction techniques followed by a limited quantitative analysis will be conducted. This preliminary study is designed to empirically study the ability to induce positive affect in a participant group. The conclusions and findings are expected to support the successful induction of positive affect. If the findings conclude that one or all methods are not effective in increasing positive affect, the primary study design may require change. 
Qualitative studies with three cohorts of 10 participants will be conducted prior to randomization of the participants into the main study arms. Audio and printed media using phone, email or postal mail as inductions methods will be piloted in the preliminary study. Positive affect induction methods to be evaluated will include word lists, humorous vignettes, social support of peers, autobiographical recall, unexpected gifts, positive feedback and affirmations.

The 30 participants will complete all forms and screening instruments. Three group meetings of 10 will participate in the qualitative study to evaluate the various induction methods and obtain satisfaction feedback and scoring on the effectiveness of each method to induce positive affect. A survey instrument will be requested to be completed for each participant in the validation study. Survey questions will include satisfaction with induction methods, self-appraisal of induction effectiveness and willingness to accept future inductions. The humor vignettes and jokes were obtained through an Internet search and www. Jokes.com for health related humor. The humor vignettes, word lists, affirmative statements and scripts for autobiographical recall and encouraging social support are detailed in the appendix.

Each individual in the pilot group will receive a pedometer and be instructed on proper use. Pilot study participants will receive the positive affect inductions daily per over a five-day period to evaluate tolerance and validate induction system operations. A survey will be conducted with each participant daily and at the conclusion of the pilot study to obtain feedback on the induction techniques and methodology procedures. 


\section{Study Participant Screening Protocol}

A copy of the informed consent form and intake questionnaire will be forwarded to the estimated volunteers $(n=290)$ prior to the intake and screening session interview. Study participants will be invited to schedule a 30 -minute appointment for intake and screening $(n=200) .200$ or $80 \%$ of the 290 volunteers are estimated to survive the volunteer and initial paper survey screening process from the original sampling frame of 9,700 females (see Figure 4).

Informed consent will be obtained from each volunteer at the screening session. The study investigator with research assistants will conduct the intake and screening sessions. Potential participants will complete Internet accessed surveys for the intake and screening sessions in the presence of research team staff. A research assistant and the study director will review all surveys and response data.

The 180 participants who are filtered and remain viable volunteers after screening and informed consent will be available for randomization. The randomization process is expected to result in $90 \%$ retention of volunteers $(n=141)$.

Thus, 141 volunteers will be available and result in the two treatment and one control arm of 47 participants each ( 3 arms $\times 47=141$ ). The estimated treatment and control arms with 47 participants are projected to result in 40 study participants who complete the study and all final measurements. The sample size calculation methodology is provided in a subsequent section. 
Individual screening sessions will be scheduled after work hours across a 1-week period, 5:30 pm - 8:30 pm Sunday through Thursday for 5 consecutive days and 9:00 am to 4:00 pm on Saturdays. This schedule provides 22 clock hours of available screening appointments. Rooms in a quiet area of a community or office practice location with privacy will be used. 71 screeninghours are estimated to complete the screening and intake process. The volunteers who have survived the inclusion and exclusion phase will be scheduled. Each screening session is estimated to require 30 minutes of evaluator interaction. The projected no-show rate for appointments is $20-25 \%$. Eight-intake staff for each scheduled hour of intake screening is planned. Four staff available for each of the 22 clock hours of screening, allows for 176 appointment slots across 88 hours, which exceeds the estimated 71 examiner hours and allows for scheduling inefficiency. The principal investigator and eight research assistants (M.S. or M.P.H. or M.S/M.P.H. students) will conduct the screening, intake and initial evaluation sessions.

The flow of each volunteer from initial contact through to randomization includes the following sequence.

1. Nominated and/or volunteer through personal medical provider or postal mail recruitment. 3,000 medical provider recruitment, 7,000 postal mail recruitment $(n=10,000)$

2. Initial demographic form completed and forwarded to study investigator $(n=970)$ 
3. Initial inclusion criteria met, surveys and informed consent forms forward to volunteers $(n=290)$. Pilot and validation study group $(n=50)$ is segregated for qualitative analysis of the study protocol.

4. Surveys completed, screening criteria, exclusion criteria examination of each participant, baseline measurements reviewed by examiners including behavioral theory variables, physical activity through pedometer steps and physical activity through self-reported recall. $(n=200)$.

5. Randomization of two treatment and control groups $(n=180)$

Prior to randomization, all participants are screened to exclude those with medical disorders or behavioral issues that make them unsuitable for the study. The rationale for exclusion criteria is provided in a subsequent section. Prior to randomization, the study director will review the screening survey responses and attest to suitability for randomization.

\section{Randomization}

It is ethically appropriate to randomize willing participants to treatment approaches that include a variety of strategies including behavioral therapy, positive affect induction, and daily telephone contact. Previous research protocols have using these treatment methods have not reported any harm or adverse impact on study participants(Isen, Daubman et al. 1987; Wing and Greeno 1994; Wing, Jeffery et al. 1996; Vanwormer, Boucher et al. 2006; Isen 2008; Estabrooks, Shoup et al. 2009). 


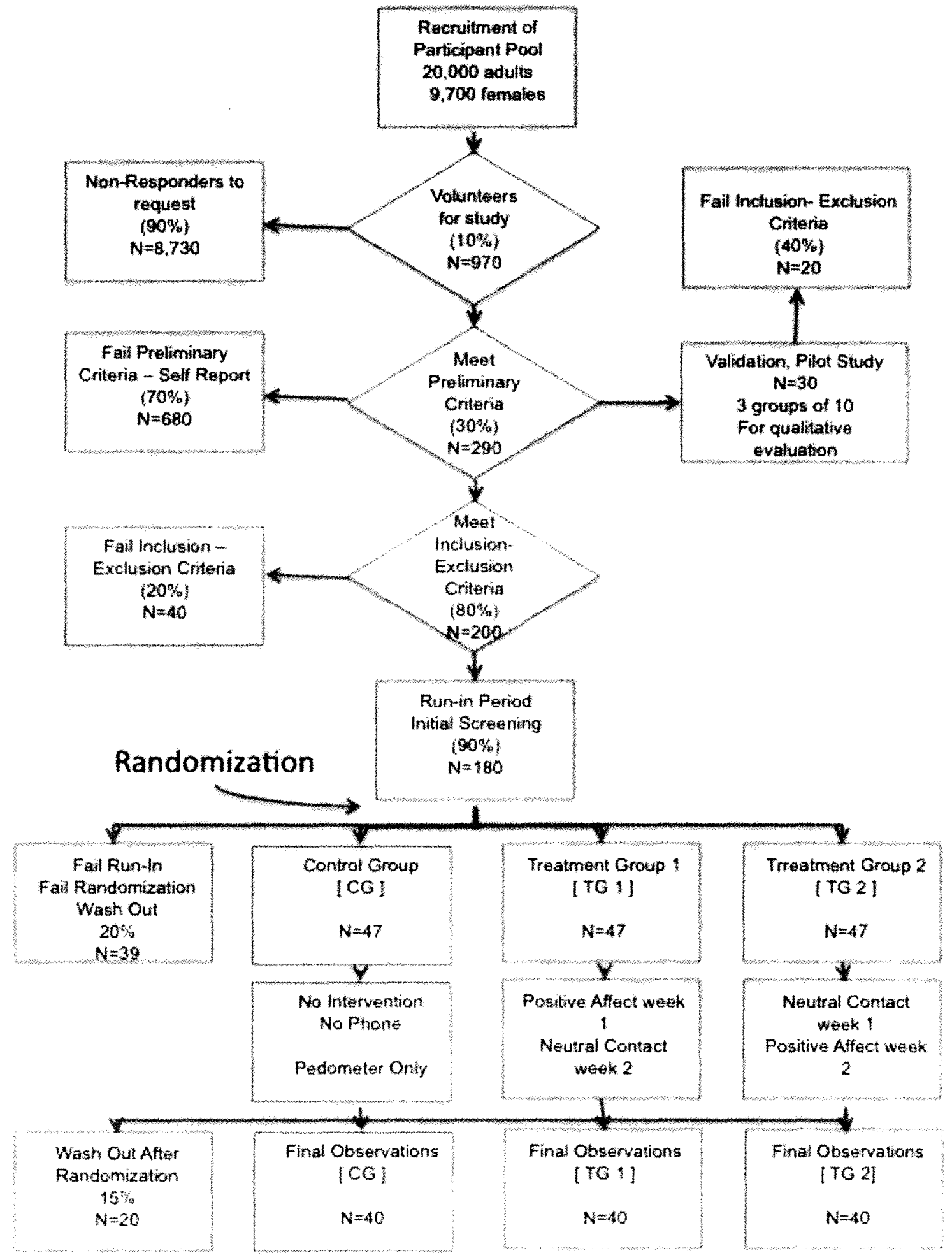




\section{Participant Selection Criteria}

Make-up of the study population will be guided by the following criteriaInclusion Criteria

$30 \%$ of the initial volunteers are expected to meet the BMI criteria. $80 \%$ of the volunteers who meet the BMI criteria are projected to meet all of the following inclusion criteria, see Figure 4.

- Agree to randomized treatment assignment

- Female

- Calculated BMI $27 \mathrm{~kg} / \mathrm{m}^{2}$ to $35 \mathrm{~kg} / \mathrm{m}^{2}$

- Current physical activity less than recommended levels.

- Able to provide written informed consent to participate

- Possess a cell phone for daily contacts

- Able to comply with study procedures

- Pre-Menopausal (intended to reduce hormonal variance as a result of menopause)

\section{Exclusion Criteria}

- Unwilling or unable to provide written informed consent

- Diagnosis or pharmacologic treatment for-

- Life threatening illness

- Limiting physical disabilities or handicaps impacting physical activity

- High-risk co-morbid conditions (uncontrolled hypertension, psychosis etc.)

- Pregnancy or intent to conceive pregnancy within the next 6 months. 
- Active diagnosis or facility admission within the previous 12 months for depression or treatment with anti-depressant pharmacotherapy or presence of depressive symptomology.

- Travel or vacation plans that inhibit full participation

\section{Delimitations}

Delimitations for the proposed study include geographical residence distance not to exceed 20 miles to the study locations; females, pre-menopausal, approximate BMI $27 \mathrm{~kg} / \mathrm{m}^{2}$ to $35 \mathrm{~kg} / \mathrm{m}^{2}$, no confounding medical conditions.

\section{Limitations}

The availability of cell phone network access, quality of phone communications and potential malfunction of the computer generated call system may limit the effectiveness of intended treatments. The participant willingness to accept and listen to scheduled calls is a potential limitation. Applied setting circumstances such as a massive failure of the cellular phone system as a result of a natural disaster or catastrophic system failure are potential limitations. Any of these factors may impede the treatments designed for delivery through the phone system.

\section{Study Design}

The study design and methodology is illustrated in Figure 5. The experimental treatment groups ( $n=47$ in each group) will receive 12 periods of interaction (TG1 and TG2): Two daily phone interactions six days per week (MSa), in each weekly period are planned. Positive affect induction will be included in all interactions in TG1 during week one, and in TG2 during week two. The 
second treatment group ( $n=96$ ) will receive the same number and duration of interactions, without any positive affect influence (TG2) during week one. Physical activity for TG1 and TG2 will be tracked through the use of a pedometer and automated collection of daily steps. A control group $(n=47)$ not receiving any treatment will participate in the two-week program (CG). The control group is designed to mimic normal care in the community. Subjects in the treatment and control groups will be evaluated at baseline and after two weeks of treatment, for variables of interest and outcome variables. Pedometer steps will be uploaded weekly. All participants will receive a booklet with promotional and knowledge content on physical activity.

Treatment Group One (TG1). TG1 is the cohort that will receive the daily phone calls with positive affect induction through humor and affirmation treatments in week one.

Treatment Group Two (TG2). TG2 is the cohort that will receive the identical interactions and treatments as TG1 with the exception that phone call interactions will be neutral health related messages, without any positive affect inductions in week 1 , with positive affect inductions starting in week two.

Control Group (CG). The CG is intended to serve as the treatment group that mimics typical community intervention activity. The CG will not receive any phone treatments. Participants randomized to the $\mathrm{CG}$ will receive a pedometer and instructions for pedometer use and a physical activity instructional booklet. CG participants will not receive any additional education or interventions from the research team. 
The rotation of the two treatment arms from positive affect treatment during week one to neutral treatment in week two for TG1 is a characteristic of a crossover study methodology. The crossover procedure is designed to hold constant the variation between individuals and allow participants to serve as their own controls for comparison between the treatment week and the neutral or control week. A concern for carryover of the treatment effect is not a consideration for this study design (Gordis 2004). The literature is informative on this issue, with no longer lasting effect on emotional scores with less than three weeks of daily treatments (Fredrickson and Branigan 2005). A third cohort has been included in the planned methodology. The third arm of the study is designed to act as a control that does not receive phone call treatments. The emotional scores of the PANAS instrument in the control group can help control for any historical or reporting bias in the data. 


\section{Figure 5. Study Design and Methodology}

\begin{tabular}{|c|c|c|c|c|c|c|c|c|}
\hline & \multicolumn{3}{|c|}{ Pre Treatment } & \multicolumn{5}{|c|}{ Week 1} \\
\hline & Randomization & Phy Activity & Baseline \& & Dally & 4 time/day & Daily & Pedometer & Weekly \\
\hline & & Booklet & Screening & Messages & Pos Affect & PANAS & & PANAS \& \\
\hline & & & & & & & & Physical Act \\
\hline TG1 & $\mathrm{R}$ & $\mathrm{X}$ & 0 & $\mathrm{X}$ & $\mathrm{X}$ & 0 & $\mathrm{X}$ & 0 \\
\hline TG2 & $R$ & $x$ & $\mathrm{O}$ & $\mathrm{X}$ & & 0 & $\mathrm{X}$ & $\mathrm{O}$ \\
\hline \multirow[t]{4}{*}{ CG } & $\mathbf{R}$ & $\mathrm{X}$ & 0 & & & & $\mathrm{x}$ & $\mathrm{O}$ \\
\hline & & \multicolumn{6}{|c|}{ Week 2} & Saturday \\
\hline & & Daily & Daily Msgs & Daily & Pedometer & Weekly & Collect & Final \\
\hline & & Messages & Pos Affect & PANAS & & $\begin{array}{l}\text { PANAS \& } \\
\text { Physical Act }\end{array}$ & $\begin{array}{l}\text { Pedometer } \\
\text { Steps }\end{array}$ & Outcomes \\
\hline TG1 & & $\mathrm{X}$ & & 0 & $\mathrm{x}$ & 0 & 0 & 0 \\
\hline TG2 & & $\mathrm{X}$ & $x$ & 0 & $x$ & 0 & 0 & 0 \\
\hline CG & & & & & $\mathrm{x}$ & 0 & 0 & 0 \\
\hline \multicolumn{2}{|c|}{ Baseline and Screening } & \multicolumn{7}{|c|}{$\begin{array}{l}\text { Med Hx, CES-D, SF-36, PAR-Q, Steps, PAR- } 7 \text { day recall, Physical Activity- Steps, } \\
\text { PANAS, Self Efficacy, Behavioral Staging }\end{array}$} \\
\hline \multirow{2}{*}{\multicolumn{2}{|c|}{ Daily Messages }} & \multirow{2}{*}{\multicolumn{7}{|c|}{ Text (SMS) or email contact at $10 \mathrm{am} 2 \mathrm{pm}, 4 \mathrm{pm}, 7 \mathrm{pm}$. }} \\
\hline & & & & & & & & \\
\hline \multicolumn{2}{|c|}{ Daily Msgs + Positive Affect } & \multicolumn{7}{|c|}{ Daily messages include positive affect induction } \\
\hline \multicolumn{2}{|c|}{ Daily PANAS } & \multicolumn{7}{|c|}{ Internet Survey Apptication at $1: 00 \mathrm{pm}$} \\
\hline \multicolumn{2}{|c|}{ Weekly Activity } & \multicolumn{7}{|c|}{ Pedometer steps uploaded, PANAS, PAR- 7 day recall , Physical Activity-Steps } \\
\hline Final Outc & & $\begin{array}{l}\text { SF }-36, \text { Steps } \\
\text { PANAS, Self }\end{array}$ & $\begin{array}{l}\text { S, PAR- } 7 \text { da } \\
\text { IEfficacy, Be }\end{array}$ & $\begin{array}{l}\text { ay recall } \mathrm{Ph} \\
\text { ehevioral St }\end{array}$ & $\begin{array}{l}\text { yssical Activi } \\
\text { taging }\end{array}$ & ity-Steps, & & \\
\hline
\end{tabular}

\section{Measurement Methods and Instrumentation}

Positive Affect

\section{Emotion Scores - PANAS}

Positive affect as measured through the PANAS scale is the study's primary dependent variable. Participants will record emotional state (PANAS) at intake, the conclusion of weeks one and two treatment periods and at a final outcome evaluation. The time frame in which participants will respond provides for a measure of the previous seven days. Participants will also record daily emotional states at the evening, 8:00 pm phone call through the IVR system.

The PANAS scales are a 10-item mood negative and 10-item mood positive affect response instrument (Watson, Clark et al. 1988). The scales are reported to display adequate internal stability and validity. The 10 labels for 
positive emotional components in the PANAS are: attentive, interested, alert, excited, enthusiastic, inspired, proud, determined, strong and active. Each emotional component can be rated on a scale from one to five: very slightly or not at all, a little, moderately, quite a bit, or extremely. The responses are scored from very slightly (1) through extremely (5). The total possible score for the positive affect scale is 50 points $(10 \times 5)$. Average daily positive mood means $(x=31.72, S D=7.38)$ and alpha reliability scores $\alpha=.90(n=657)$ have been documented (Erez, Isen 2002). The daily scores for the positive score only will be the primary variable for statistical analysis as the dependent variable. The negative affect score provides a comparative score to evaluate discriminant validity. Weekly scores will be a sum of the six daily scores.

Positive Affect Induction.

The positive affect treatments will be delivered through phone contacts. Positive affect has been induced in laboratory conditions with humor, emotional movie or audio clips emphasizing positive emotions, positive word association exercises, personal affirmations and small unexpected gifts to participants (Isen, Daubman et al. 1987; Isen 1998). The positive affect induction methods for the treatment groups will be conducted through audio files via the telephone. Humorous vignettes, word lists, affirmative statements that have been previously validated by a comparable demographic participant group in a pilot study, will be induced through the telephony system. The final set of selected induction methods will be selected by a pilot study of 30 volunteers. The recruitment of this pilot study group will occur prior to randomization and has been described in 
a previous section (see Figure 4). The effects of similar inducements on positive affect have been observed to last commonly for 10-30 minutes with longer-term impact on decision making with daily treatments (Roehm and Roehm 2005; Isen 2008). Individuals in the two treatment groups will receive a scheduled two phone calls per day, six days per week, Monday through Saturday. The calls will be scheduled at 2:00 pm and 8:00 pm. The sequence of steps and affect induction is illustrated in Figure 6.

\section{Physical Activity}

\section{Physical Activity Level, Steps per Day}

Physical activity is measured objectively by a mechanical pedometer (Merom, Rissel et al. 2007; Clemes and Parker 2009). The pedometer is battery powered and maintains approximately 14 days data. Each study participant will receive the pedometer at the conclusion of the screening examination. The step data will be collected on four consecutive Saturday's. The four Saturday collection points are the Saturday prior to the first week of treatment, at the end of the first week, the conclusion of week two and one week after the conclusion of the study. Data will be transferred via a USB cable to the database storage of the commercial pedometer vendor on these four days at a single location. Individuals will bring the pedometer in for uploading. The uploading process takes approximately one-minute. The pedometer records steps within a 24-hour period for each calendar day. A single missed upload is not critical as the pedometer can maintain step storage for 14-days. The data storage and electronic transmission capability reduces reporting error through the removal of 
participant requirements to maintain logs for self reported activity. Each step is recorded when the hip drops and rises during walking. Although the pedometer will collect all steps, a limitation is the intensity of physical activity and the exact nature of the activity that records each hip movement. Vigorous physical activity at a running pace of 10 miles per hour, over 1,000 steps is indistinguishable from 1,000 steps at a pace of 4 miles per hour. Participant adherence of wearing the pedometer at all times is critical for accurate collection of daily step activity. Participants will upload the pedometer data at a designated location on the Saturday of each week. The data will be stored on the commercial pedometer vendor server and database until the end of study download is completed.

\section{Physical Activity - Seven-Day Recall}

Participants will be asked to complete a recall diary of physical activity for the previous seven-days on Saturday's during the study period. The instrument for this data collection is the tool validated by Sallis and colleagues title, SevenDay Physical Activity Recall (PAR) (Sallis, Haskell et al. 1985). The PAR instrument has been widely used since the 1980's and includes a methodology for estimating energy expenditure in standardized units of metabolic equivalents of task (MET). The calculated METs from the PAR will be the variable used for analysis.

The PAR collection is intended to account for all physical activity performed by participants. The self-reported physical activity may increase the sensitivity of changes in behavior, for activity that may not be accounted for in daily steps through pedometer readings. A comparison between pedometer 
steps and PAR activity is planned to evaluate any potential influence between affect, activity and self-reported activity.

The PAR will be administered on four occasions. The collection days will be scheduled on the Saturday preceding the start of the treatment period, the two Saturdays in each treatment week and the final recall one-week post treatment.

\section{Physical Activity - Walking}

Participants will be asked to increase daily physical activity as measured by steps. Individuals who average less than 7,000 steps per day during the runin period will be asked to increase activity to 7,000 steps per day. Individuals who average more than 7,000 steps per day during the run-in period will be asked to increase activity to 10,000 steps per day or 3,000 additional steps if activity average exceeded 10,000 steps.

\section{Steps Completed}

An aggregate count is calculated for each 24-hour period 0001 to 2400 daily. Pedometers are registered to each participant and linked through the device serial number. The activity file with all participants daily step count will be obtained from the vendor at the conclusion of the study for analysis and integration with other study variables. Intra-group analysis including interaction between the treatment calls, PANAS scores and steps will be analyzed using this variable.

Steps per Week Calculation

The total steps for each seven-day period Monday through Sunday will be summed and coded as weekly activity as well as each daily step count. 
Comparisons between groups (treatment vs. neutral/control) and within groups (treatment week vs. neutral week) will be analyzed with this variable.

Demographic and Screening Variables.

Demographic and screening variables will be collected during the screening interview. Individuals will complete the survey via Internet application. The instruments will collect general demographic variables including gender, date of birth, race, socio-economic status (Featherman and Hauser 1976; Gallo and Matthews 2003; LaVeist 2005), occupation, medical history, age (calculated), CES-D cut-off score and subscale scores, and PANAS affect score. Body Mass Index (calculated)

The weight and height measurement self-reported at screening will be used to calculate BMI. This calculated BMI will be used to determine eligibility in accordance with the study inclusion criteria. BMI will be calculated with the Quetelet Index (Bray 2004), reported as kilograms divided by meters squared, $\mathrm{kg} / \mathrm{m}^{2}$. The weight recordings in pounds will be converted to kilograms. Height in feet and inches will be converted to meters.

\section{$\underline{\text { Socio-economic Status }}$}

Financial profile and occupation will be used to calculate a socio-economic status (SES) index. The two sections are weighted with an index scaled from 0 to 99 , calculated from normative research analysis (Nam and Powers 1983). A mean score will be created from the two separate sections as a new calculated variable for the SES index score. The SES index is based on general principles outlined in the literature (Lillie-Blanton and Laveist 1996; Gallo and Matthews 
2003; LaVeist 2005; LaVeist, Thorpe et al. 2007). The SES score is unique and has been developed for this study. This interval score is based on the conclusions and findings outlined by LaVeist. This interval score is designed to be practical for descriptive and correlation analysis.

The scoring methodology is intended to provide discriminant results for economic well being that displays increased accuracy as positioned by LaVeist. Scoring for the 99-point financial profile will follow; business owners garner 29 points, and affirmative responses to the remaining five questions are valued at 14 point each; savings account, checking account, own real estate, own stocks/bonds/mutual funds, own certificates of deposit.

Respondents are requested to provide a descriptive label for their occupation. A 100-point scale has been previously published that established a relationship between occupation and social status (Reiss 1961; Nam and Powers 1983). The occupational score is combined with the financial profile and a calculated mean will become the SES Index variable for subsequent analysis.

\section{PAR-Q Pass}

The physical activity readiness questionnaire (PAR-Q) has been designed and updated to screen individuals for risk of a cardiac injury and restrict low, moderate or vigorous physical exertion (Thomas, Reading et al. 1992). Any affirmative response is considered a trigger for high-risk status and subsequent physician clearance prior to initiation in any physical activity regimen. Volunteers who do not obtain physician clearance will be excluded from participation in the study. 
SF36/MOS score.

Quality of life is assessed through the use of the Medical Outcomes Study Standard Form Health Survey (SF-36). This is a 36-question instrument that produces subscales in physical function, role function-physical, role functionemotional, bodily pain, general health, social function and psychological wellbeing/mental health, vitality health transition, and side effects checklist. This widely used instrument is comprehensive in scope and has been used in previous studies. The SF-36 has a normative database with previous validation with reported stable psychometric properties. Life Orientation Test (LOT).

The LOT is a 12-question instrument that assesses expectancies for positive and negative outcomes. Four items are positive statements, four items are negative statements and four items are neutral statements. The positive and negative statements are scored on a five-point response scale. Negative statements are reverse scored. Neutral statements are not scored. The range of scores for the eight statements is 0 to 32 , the higher scores associated with higher optimism (Scheier, Carver et al. 1994).

Behavioral theory measures.

A survey will record components of behavioral change intent and status using the Health Action Process Approach (HAPA) theory as the model of choice (Schwarzer 2008; Schwarzer and Luszczynska 2008). The components for behavioral staging and evaluation include self-efficacy (Bandura 2001; Dishman, Motl et al. 2005; Blanchard, Fortier et al. 2007), outcome expectancies, risk 
perception, planning tasks, action tasks, maintenance tasks (Dishman, Motl et al. 2005; Schwarzer 2008).

Center for Epidemiologic Studies, Depressed Mood (CES-D)

Due to the potential adverse impact on compliance in future data collection, individuals who exceed a cut-off score of 16 points and are therefore considered symptomatic of depression (Radloff 1977), will be excluded from participation in this study. This exclusion includes individuals with a current or past history of bipolar disorder, and cyclothymic and dysthymic disorders. In addition, individuals evidencing present or past history of psychotic symptoms as part of a depressive disorder will be excluded from the study, without exception. Individuals who have a history of major depressive or mood disorder who have been mood and weight stable for at least 6 months on antidepressant medication will be considered, but will be excluded unless physician guidance encourages participation. Factors to be considered when evaluating the eligibility of depressed participants for inclusion in the study are history of study appointments, prominence and severity of depressive symptoms, impact of depressive symptoms on daily activities, expressed interest in participation, and absence of in-patient treatment of the disorder. All these issues are addressed in the screening instrument.

Depression will be screened by use of the CES-D as described above. Individuals who develop depressive symptoms during the trial will be referred to their medical provider for evaluation. Any individual expressing suicidal thoughts or ideation during screening will be referred to their medical provider. No 
individual expressing suicidal ideation or thoughts will be randomized into the study. Suicidal ideation will require immediate study withdrawal of the participant from further study participation.

\section{CES-D Score Calculation}

The respondent's answers are used to calculate a score and then compared to a previously established score that provides an indication for depression, with a maximum score of 60 . Twenty symptomatic questions are presented with four response options: rarely or none of the time (less than 1 day), some or a little of the time (1-2 days), occasionally or a moderate amount of time (3-4 days), most or all of the time (5-7 days). The lowest acuity option is scored at zero, and the subsequent responses increase in score value, 1-2 days -1 point, 3-4 days -2 points, and 5-7 days -3 points. Previously published means in a white population ranged from 7.94 to 9.25 . Four CES-D subscales including depressed affect, positive affect, somatic activity and interpersonal factors, will be calculated and analyzed for potential change as a result of interventions (Radloff 1977). The total score for psychiatric treatment patients was 24.42. Volunteers that score equal to or above a score of 16 will be considered for exclusion from consideration as a study participant.

Telephone Contact.

The utility of telephone contact and the induction of positive affect through an IVR system will be introduced as the treatment of interest in this study. Personal telephone contacts have been reported to be an effective behavioral counseling technique with increased efficiency compared to face-to-face 
counseling (Vanwormer, Boucher et al. 2006; Chapman, Lesch et al. 2007; Eakin, Lawler et al. 2007). The calls will be designed for participants to use the numeric touch pad of the phone or interactive voice response. If the participant does not answer the call, a voice message will be left that the participant can return and receive the intervention interaction. The calls allow the participant to receive a message that will include a positive induction for the treatment group. 
Figure 6. Telephone Call Interaction and Flow Chart

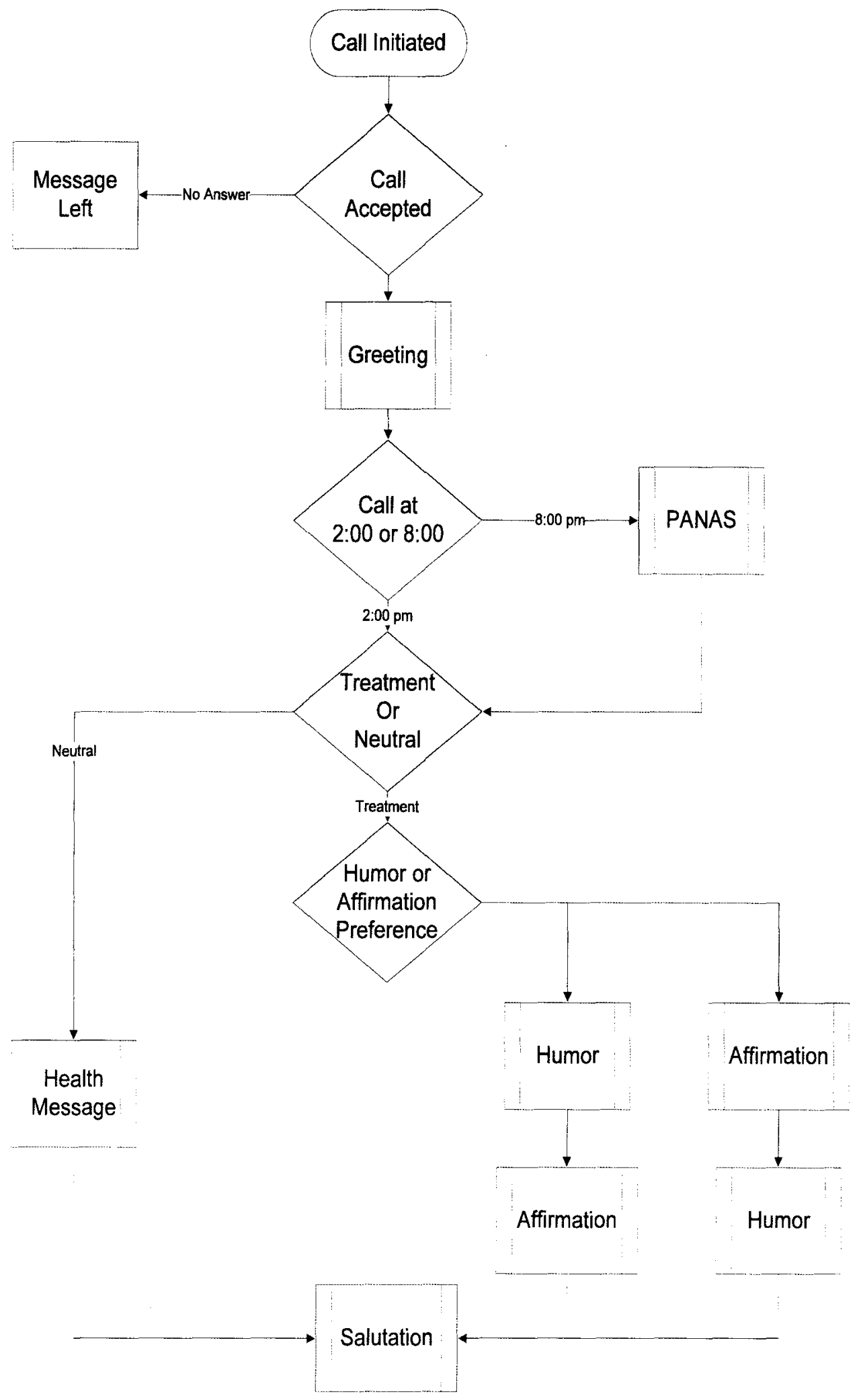




\section{Phone Calls Completed - Calculation}

An electronic record is compiled by the computer generated health dialog system for each outbound call to a participant. When the participant accepts a call and selects a decision option, the call is defined as accepted; when one of the final decision options is selected the call is defined as completed.

The number of calls completed each day $(n=2)$ and for each week $(n=12)$ will be used in a ratio calculation. Completed calls will be the numerator and the attempted/scheduled calls will be the denominator in the expressed ratio. This interval score expressed as a ratio to the hundred decimal points will be available for analysis.

\section{Measurement Challenges}

Self-reporting of positive affect has limitations. The inducement of positive affect through introspect or mindfulness can be particularly problematic given the difficulty of interpreting the feeling descriptions of the subjects (Isen and Erez 2007). A recommendation by Isen and Erez is to evaluate affect through multiple perspectives such as cognitive evidence, physiological measures and comparisons to a cohort that is expected to perform differently considering theoretical expectations (Isen and Erez 2007). The PANAS instrument used in this study includes a five-point scale for ten emotional constructs with the following discrete choices; very slightly or not at all, a little, moderately, quite a bit, extremely(Watson, Clark et al. 1988). Using synonymous terms in multiple questions can help assess the same emotional state, such as how happy, how pleased, how pleasant. When a single affective state is the focus of study, 
including additional affective states with multiple questions for each can improve the construct validity and allow for discriminant validation (Isen and Erez 2007). The use of the PANAS instrument combined with the physical activity measurement is intended to reduce potential bias of affect measurements (Isen and Erez 2007).

\section{Statistical Analysis Methodology}

Randomization

A biostatistician consultant to the study team will perform the randomization process. Each participant volunteer will be assigned a computer generated random number through the Microsoft Excel application. The sample population will be sorted in ascending order by the random generated number. The first individual will be assigned to TG1, the second to TG2, and the third to CG. This will be repeated until there are 96 participants in each arm. The initial pilot group of 30 participants will use the same procedure for random assignment.

Prior to final assignment, each study arm will be compared for potential confounding variable interactions. Demographic variables, baseline PANAS, behavioral profile variables and baseline pedometer steps will be compared using Chi-squared statistical test for categorical variables and ANOVA for continuous variables to validate random assignment. If any of these variables fail the statistical test and therefore randomization, a stratified assignment will be performed to reduce potential confounding effects. 
When the randomization and cohort assignment process is completed, participant notification will occur. Participants will learn their treatment assignment via phone from a staff member who is not involved in follow-up data collection or direct participant contact.

Study Parameters

Will the induction of positive affect through a telephone based interactive voice response system (IVR) increase positive affect in the treatment group compared to a control group? The instrument to assess this change is the PANAS.

The secondary research question becomes, will the induction of daily positive affect be an effective means of driving an increase in daily physical activity in patients in the treatment group compared to a control group in a community environment?

Null Hypotheses $(\alpha=0.05)$

Ho-1: Patients who receive positive affect inductions will not increase their daily positive affect scores on a PANAS scale instrument.

TG1 daily PANAS score means will be compared to TG2 daily PANAS score means as a joint hypothesis using regression analysis or analysis of variation (ANOVA), with two parts: $\beta_{1}=0$ and $\beta_{2}=0$. The comparison will be completed inter-group for each of the two weeks, TG1 on TG2, TG2 on TG1. A comparison intra-group for the positive affect week compared to neutral affect week will be completed using the same statistical methods, TG1-week 1 vs. TG1week 2, TG2-week 1 vs. TG2 week 2. 
The use of latent growth curve modeling, a form of structural equation modeling (SEM) of the participant's daily PANAS scores is planned. Latent growth curve modeling (LGM) is intended to permit analysis of intra-individual change over time as well as inter-individual and intergroup change over time (Preacher, Wichman et al. 2008). LGM is designed to investigate inter-individual differences in change of time in consideration of antecedents. LGM can also provide group level statistics such as mean growth rate of scores. LGM may be considered a unique model of structural equation modeling (SEM) (Preacher, Wichman et al. 2008). LGM can provide more flexibility measuring change than ANOVA (Duncan, Duncan et al. 2006). A key distinction of LGM is the ability to approximate random changes in measurement error. ANOVA is not able to accommodate complex growth and correlates of change as well as LGM (Duncan, Duncan et al. 2006).

Ho-2: Patients who are targeted for physical activity and receive positive affect induction will not yield a change in daily physical activity compared to comparable sets of control subjects. The use of physical activity as measured in daily steps may be supportive of increased PANAS scores. TG1 daily steps will be compared to TG2 daily steps as a joint hypothesis using regression analysis, with two parts: $\beta_{1}=0$ and $\beta_{2}=0$. The comparison will be completed inter-group for each of the two weeks, TG1 on TG2, TG2 on TG1. A comparison intra-group for the positive affect week compared to neutral affect week will be completed using the same statistical methods, TG1-week 1 vs. TG1-week 2, TG2-week 1 vs. TG2 week 2. 
Alternate Hypotheses $(\alpha=0.05)$

Ha-1: Patients who receive positive affect inductions will increase daily scores on the PANAS scale instrument.

Ha-2: Patients who are targeted for daily physical activity and receive positive affect influence will increase daily steps.

Sample size determination

We will be evaluating the change in PANAS scores. A mean effect size of 0.34 has been reported, across a range of positive affect studies (14 studies, 749 subjects) (Lyubomirsky, King et al. 2005). The mean PANAS score difference between positive affect treatment and control reported in multiple studies of a typical population is 2.5 to 8.3 , with reported standard deviations of 5.8 - 6.3 (Erez and Isen 2002; Roehm and Roehm 2005) for mean PANAS 10 question scores. Calculating an $(\alpha=0.05$, beta $=0.80)$, between mean difference for within participants $\mathrm{SD}=7.38$ for total PANAS scores and seeking to prove a mean difference of at least 2.5 units the minimum sample size is calculated to be 55 per arm for a crossover study design (Schoenfeld 1995). Funding limitations and the exploratory intent of this study have limited the initial sample size to 47 per study arm. The sample size in each treatment arm is estimated to reach a minimum cohort of 40 at the final outcome measurements. At the final treatment cohort of $n=40$, and a detectable difference of 2.5 units, with alpha acceptable limits for a Type I error set at 0.05 , then the power estimate of a Type II error becomes 0.68 (Schoenfeld 1995). At the final treatment cohort of $n=40$ per treatment arm, with alpha acceptable limits for a Type I error set at 0.05 , and the 
power estimate of a Type II error at 0.8 , then the detectable difference required becomes 2.93 units (Schoenfeld 1995).

\section{Outcome Measures}

\section{Primary Analysis}

The planned primary analysis of dependent variables will use the intent to treat model (ITT) (Haynes, Sackett et al. 2006). Participants assigned to one of the treatment arms at randomization will be included in the analysis, irrespective of completion or participation level. Missing data will be imputed, based on pre-randomization data and other available data. The ITT methodology is intended to produce a conservative analysis of any treatment effect. The inclusion of all participants, post randomization, regardless of the reason for nonparticipation produces the least favorable treatment effect (Haynes, Sackett et al. 2006).

\section{Outcome Measures}

The primary outcome is change in daily positive affect scores. These scores will be recorded as a self-report of emotional state over the previous day. This primary outcome will also be measured at the end of the treatment period, with a self-report of emotional state over the previous 7-day period.

Secondary Analysis

The secondary outcome includes change from baseline to outcome assessment for physical activity, recorded in step units by pedometer. Dependent Variables

- PANAS Scores 
- Physical Activity Level, steps per day

- Physical Activity Level, PAR survey instrument

Independent Variables

- Positive Affect Treatments

- Neutral Affect Treatments/daily phone calls

- Usual Care/Control

Screening Variables

- Date of Birth

- Race

- Age (calculated)

- CES-D depression score

- Physical Activity Levels, PAR (self-reported), pedometer steps

- Socio-economic index

- Physical issues that can limit activity, pre-existing or extemporaneous, PAR-Q high risk responses, lower extremity injuries that limit walking, pregnancy

Mediating Variables

- Behavioral theory measures, self-efficacy, intention to change, perceived control

- CES-D Subscales

- SF-12 Subscales

- Life Orientation Test 


\section{Safety Issues}

Screening Methods. A pilot group of 30 volunteers will be selected at random from the participant pool. Qualitative investigation of research preferences for small gifts, incentive levels, acceptance of daily calls, and humor and affirmation preferences and receptivity to positive induction will be completed. The pilot group will also be used as an initial training group for examiner training and orientation. The examiners and research assistants will validate timing, baseline measures and intake survey responses. The following safety procedures are designed to increase the safety of study participants through appropriate screening techniques and monitoring processes during the treatment period.

Physical Activity

All participants will be asked to increase physical activity as a component of overall health improvement. Participants who are sedentary are at increased risk for musculoskeletal discomfort such as delayed onset muscle soreness (DOMS), discomfort during exercise, shortness of breath, increased thirst and potentially foot blisters or irritation. Walking will be the recommended mode of physical activity for participants at a moderate to light level of intensity. Individuals who are at a physical activity level greater than sedentary will be asked to increase physical activity by an additional 3,000 steps per day. Vigorous activity will not be recommended (USDHHS 1996; Grundy, Blackburn et al. 1999) for any participant. Individuals with elevated blood pressure will be referred back to their personal physician for clearance to continue in the study 
protocol. Written approval of a participant's personal physician is required for further participation when elevated blood pressure has been documented.

The physical activity type, duration and intensity are beyond the control of study staff. Participants will be encouraged to follow established light to moderate physical activity guidelines. Periodic IVR phone contact will reinforce these physical activity recommendations. Participants will receive a brochure with information on proper physical activity guidelines including warm-up, cooldown, stretching and intensity monitoring (Borg Scale). A research assistant at the conclusion of the screening appointment will distribute the physical activity brochure.

Adverse Event Reporting.

The participants in this study will be overweight or obese and at a slightly elevated risk over the general population for morbidity and mortality. We will not track adverse events with the same intensity of a clinical Phase III drug study, but we will track Serious Adverse Events (SAEs) during the treatment period. The experience of the Diabetes Prevention Program - Observational Study at the Pennington Biomedical Research Center (Brantley 2008) provides an indication of $10 \%$ SAEs, with a higher risk for co morbid conditions than our proposed participant cohort. Thus, a much lower level incidence of SAEs is anticipated.

The rationale for tracking SAEs is to determine increased risk of the positive affect intervention and or intensive weight management treatments (future investigations). The two treatment groups receiving daily phone calls are 
the most likely to experience SAEs due to the increased frequency of interaction, albeit the expectation is for no SAEs. All groups will be monitored for SAEs.

The study director will fill out the SAE form and transmit to the Dissertation Research Chairperson within 48 hours of the reported incident.

\section{Contact with Medical Providers}

The medical care delivered by providers to participants is not expected to impact any planned intervention. Participants will be instructed to inform any medical provider of their participation during the study period when seeking medical care or advice. The study director will field inquiries from any medical provider. The treatment group interventions will be revealed or unmasked upon request of any medical provider. This will not be considered grounds for participant exclusion. The specific research questions or hypotheses will not be revealed to medical providers prior to the conclusion of the final outcome assessments.

The cooperation and support of personal physicians is a key component for participants and study success. Although physicians will be blinded to treatment arm assignment, communication on study progress and final outcomes will be made to the University of Louisville Family Practice physicians who are the source of participant referrals to the study. The study director prior to recruitment and after data analysis will complete research presentations to physicians and office staff. 
Participants will be encouraged to consult with their personal physician to obtain clearance for participation for any clinical circumstance or condition that is a concern to the participant.

\section{Study Closeout}

Participants will receive a copy of personal measures for baseline and outcome periods including pedometer steps counts. All participants will have access to health educator recommendations on continued physical activity or related health behavior programs of their own choosing at the final evaluation individual session. Participants will be informed of potential follow up studies and possible recruitment efforts.

\section{Data Safety Monitoring Board (DSMB)}

A DSMB will not be designated for this study. All safety issues will be reported to the study director within 24 hours of any incident. The Study Director will consult with the Faculty Advisor/Principal Investigator within hours verbally and in no more than 24 hours with a written incident report. The Principal Investigator will inform the IRB Director about any serious safety incident.

\section{Benefits}

The delivery of benefits to participants is expected but cannot be guaranteed. Anticipated benefits for all participants are an increased awareness and new knowledge of physical activity benefits. The benefit of positive affect induction in this study is hypothesized but unknown for study participants. The participation of individuals in this study will help identify the utility and influence of positive affect in a practical setting for health behaviors. The lessons learned 
through this study are expected to provide insight and yield improved techniques for follow-on and larger scale studies of a similar nature.

Participants will receive monetary incentives up to $\$ 50.00$ total for completion of baseline and final outcome examinations in an attempt to compensate for time and transportation expenses.

\section{Blinding, Unblinding, Masking}

We will maintain confidence wherever feasible and practical to limit the knowledge of treatment group assignment in an effort to reduce any potential bias. The participants will know the treatment group assignment and be informed of the expected interventions. Participants will not be informed if they will receive positive affect induction, beyond the general orientation and informed consent during screening and baseline interactions. Participants in TG1 and TG2 will be informed of the telephone contact, but not the intended intervention of positive affect or neutral affect.

The research staff and evaluators who conduct the initial screening, baseline assessments, final assessments and data handling will be blinded to participants' treatment group assignments. The data collected in the baseline and final assessment will be blinded to all staff interacting with participants.

Each individual participant will receive a copy of his or her initial assessment and final assessment measures at the conclusion of the study period. These results will be distributed at a group session or mailed to the individual at their home address of choice. 


\section{Exploratory Study Summary}

The exploratory study was planned to validate positive affect induction methods. A qualitative study of induction techniques followed by a limited quantitative analysis was conducted. This preliminary study was designed to investigate the ability to induce positive affect in a participant group in an applied environment. The a priori hypothesis is that the conclusions and findings will support the successful induction of positive affect in an applied setting. These findings will shape the research design and methodology for a planned follow-up main study.

A group of 30 volunteer participants were obtained through multiple recruitment methods. Three qualitative feedback sessions were conducted to document input and opinions on planned induction techniques and procedures. The first group was shown each communication message on a projector screen. Individuals graded each message on a five-point scales. A=most preferred, $B=$ somewhat preferred,$C=$ neutral preference,$D=$ not preferred, $E=$ least preferred. Individual scores were recorded anonymously through an electronic audience response system. The proposed health messages were organized in three segments; general health messages, affirmation statements and humor messages/jokes. Approximately 80 communications in each of the three segments were presented to the first of three sessions $(n=5)$. The messages were presented as a group and feedback obtained on each segment before moving to the next message type. The 25 highest scoring messages, the 5 lowest scoring messages and 5 random messages were grouped after the first 
group session and these 35 messages were evaluated by groups two and three. The 24 messages in each segment with the highest scores were identified for use in the proposed follow-up study. Discussion and feedback after each session was obtained to develop common themes or rationale for message scoring.

Planned recruiting methods yielded less than 15 study volunteers after eight months of recruitment. The initial qualitative session to obtain feedback on potential communications was completed with five participants. The recruiting protocol was modified to a more direct contact. Emails were distributed to contacts in employee populations and personal recruiting or networking techniques were designed and executed. This resulted in the second and third sessions of 12 and 13 participants.

The evaluation, and scoring of messages were sorted and used to select the 24 most preferred messages in each of the three categories; general health, affirmations, and humor. Commentary and feedback was documented in each session. Common word and trend analysis of comments were used to conclude that communication via phone, email, text are acceptable. Phone contact was considered to be the least acceptable by many study participants.

The completion of the survey instruments did not reveal any negative comments or experiences with the exception of the physical activity recall survey. The completion of the seven-day physical activity recall was the most challenging survey. Participants indicated a degree of frustration over an inability to recall the time and duration of activity for the previous 7-days. The design and 
distribution of a log-sheet for hand completion prior to the on-line survey application data entry mitigated some of the stated frustration among participants.

The use of the pedometers / accelerometers was executed without difficulty. Missing data on days when participants temporarily lost the device, or forget to wear the device were noted. The missing data fields were few and did not jeopardize the data analysis. Participants commented on the novelty of the accelerometer and enjoyed the use of the device during the study.

The distribution of messages through the various communication channels was completed without any major obstacles. The email and text distribution of messages were the least difficult to design and deploy. The design and programming required for the operation of the automated interactive phone system required a large amount of resources. The time to develop the architecture and program the computer-generated system exceeded ninemonths. The ability to connect with participants at each scheduled contact exceeded $90 \%$. The timing of the message receipt was not consistent. Participants were unavailable for phone or email contact and did not always receive messages at the target delivery times. The use of multiple channels appears for each scheduled contact provided flexibility for participants to select the most convenient communication medium. 


\section{CHAPTER IV \\ MAIN STUDY RESULTS}

\section{Introduction}

An exploratory study was previously completed to validate positive affect induction methods. A qualitative study of induction techniques followed by a limited quantitative analysis was performed. This preliminary research was designed to study the ability to induce positive affect in a participant group. The exploratory study was intended to increase the accuracy of the induction methodology and serve as a validation of the screening procedures and other methods. Participant tolerance for the daily contact and pedometer use was also validated. The findings and conclusions from the exploratory study resulted in modifications to the main study methodology. The results, discussion and conclusions from the exploratory study are included in Appendix 1.

The influence of mood induction on positive affect was measured through completion of the self-reported Positive and Negative Affect Survey (PANAS). The PANAS was accessed through an Internet survey application. A link for the daily completion of the PANAS in the main study was distributed at 1:00 pm on each of the weekday treatment periods over the two-week observation period. A seven-day recall PANAS was completed on Saturdays by participants at the baseline-intake prior to the treatment period and on Saturdays of both week one and two. 
Each participant in the main study received an accelerometer/pedometer and instruction on proper use. Participants were asked to wear their accelerometer/pedometer throughout the two-week period after distribution. Study participants received the positive affect inductions Monday through Friday for one week in a two-week observation period. A single humor message/joke was distributed to participants four times per day over the five-day period. The humor vignettes and jokes were obtained through an Internet search and www. Jokes.com for health related humor. Messages were delivered through email or text (SMS) at 10:00 am, 2:00 pm, 4:00 pm and 8:00 pm in the participant's time zone. Respondents provided the preferred email addresses and/or phone numbers for humor text message distribution. General health messages were distributed to participants in the alternate week of the two-week study period and were referred to as neutral treatments.

\section{Participants}

\section{Recruiting and Selection}

The main study volunteers were asked to complete forms and screening instruments through an Internet based survey application. Study volunteers were recruited from the workforces of large employers in various locations across the United States. The recruitment period ran for 3 months. Volunteers accessed the initial data form application 157 times and 53 individuals completed the form. An internal access corporate social media site obtained 41 participants. These two volunteer pools combined, produced an initial 50 volunteers who agreed to proceed and initiated the informed consent and research authorization process. 
A total of 36 participants completed the main study baseline survey. Six individuals withdrew from the study. Figure 1 illustrates the study population and study arm composition tree.

\section{Demographics and Randomization}

Volunteers who completed the informed consent and research authorization process and met the criteria who successfully completed the baseline survey were assigned to one of two volunteer pools. Individuals who met all inclusion and exclusion criteria were assigned to the first pool $(n=19)$. Individuals who did not meet the BMI or age criteria, but met all other inclusion and exclusion criteria were assigned to the second pool $(n=17)$. The demographic profile and other personal characteristics are displayed in Table 1. The study cohort was generally White American, married, college educated, employed full-time. The participants in the first pool were assigned to the two treatment groups through a randomized assignment process. Each individual in the first pool was assigned random number generated through a statistical software application (SPSS 2008). The file was sorted on the random number and individuals were then sequentially assigned group one $(n=10)$ and group two $(n=9)$. 
Figure 7.

\section{Cohort Tree Diagram - Main Study}

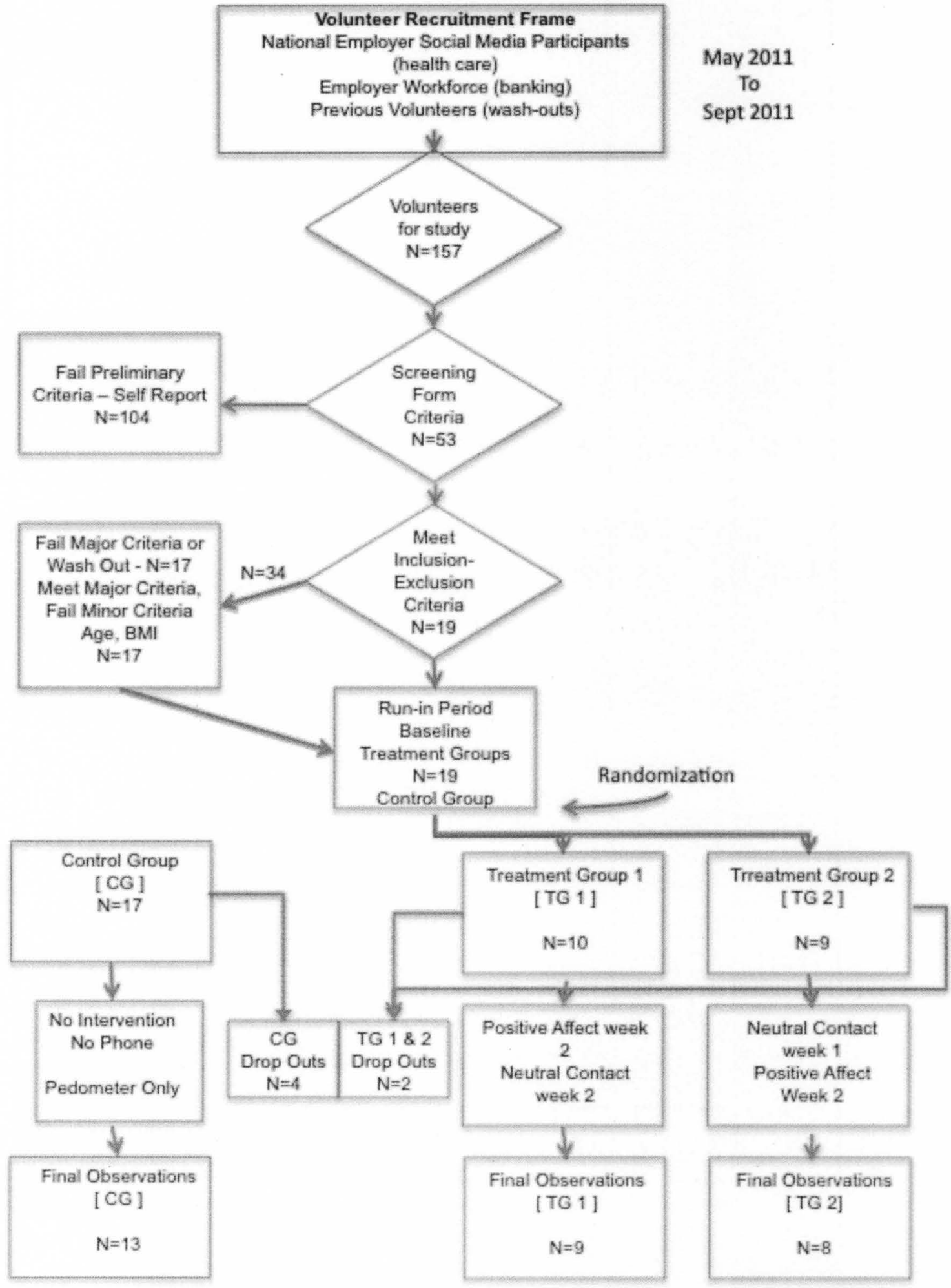


Table 4.

\section{Demographics}

\begin{tabular}{|c|c|c|c|}
\hline Demographics & All Groups & & $\mathbf{n}$ \\
\hline Race (\% Caucasian) & $75 \%$ & & 27 \\
\hline Married & $75 \%$ & & 27 \\
\hline Bachelor / Graduate Degree & $62 \%$ & & 22 \\
\hline Employed & $92 \%$ & & 33 \\
\hline Household Income > \$60K & $91 \%$ & & 32 \\
\hline No Chronic Conditions & $56 \%$ & & 20 \\
\hline Mean Age & 41.3 & 10.6 (SD) & 36 \\
\hline \multicolumn{4}{|l|}{ Medical Conditions } \\
\hline Hypertension & $12 \%$ & & 4 \\
\hline Hyperlipidemia & $12 \%$ & & 4 \\
\hline Previous History of Cancer & $3 \%$ & & 3 \\
\hline Other Chronic Conditions & $3 \%$ & & 3 \\
\hline
\end{tabular}

Table 5.

\section{Cohort Characteristics}

\begin{tabular}{|c|c|c|c|c|c|c|c|}
\hline & \multicolumn{2}{|c|}{ Group 1 (T1) } & \multicolumn{2}{|c|}{ Group 2 (T2) } & \multicolumn{2}{|c|}{ Group $3(\mathrm{C})$} & \multirow{2}{*}{$\begin{array}{c}\mathrm{p} \\
\text { value }\end{array}$} \\
\hline & Mean & (SD) & Mean & (SD) & Mean & (SD) & \\
\hline \multicolumn{8}{|l|}{ Measure } \\
\hline Cohort Size n- & & & & & & & \\
\hline Conort Size, $n=$ & $\frac{9}{296}$ & (70) & $\frac{8}{278}$ & (19) & $\frac{13}{248}$ & $(51)$ & \\
\hline SES Score & 87.7 & $(8.7)$ & 81.4 & $\frac{(1.0)}{(9.1)}$ & $\frac{24.0}{79.4}$ & $\frac{(3.1)}{(14.9)}$ & 0.48 \\
\hline CESD Score & 2.2 & $(2.8)$ & $6.3^{\prime}$ & $(5.7)$ & 9.8 & $(6.5)$ & $0.01^{*}$ \\
\hline PANAS, Positive & 37.7 & (4.9) & 37.8 & $(7.7)$ & 37.3 & (9.4) & 0.95 \\
\hline PANAS, Negative & 15.1 & $(7.8)$ & 11.3 & $(1.3)$ & 15.1 & (6.3) & 0.19 \\
\hline Life Orientation Score & 19.1 & (3.1) & $17.6^{\prime}$ & (3.2) & 12.9 & (2.0) & 0.18 \\
\hline Confidence Score & 8.0 & (1.3) & $7.9^{\prime}$ & $(1.3)$ & 8.4 & $(1.8)$ & 0.68 \\
\hline SF-36 Score & 84.4 & $(8.4)$ & $80.6^{\prime}$ & $(9.2)$ & 85.0 & $(15.0)$ & 0.55 \\
\hline Age & 37.7 & $(8.8)$ & $38.1^{\prime}$ & $(4.8)$ & 45.8 & $(12.8)$ & 0.22 \\
\hline${ }^{*}$ CESD significant $d$ & veen & & & & & & \\
\hline
\end{tabular}




\section{Characteristics}

The profile of the participants in the two treatment groups and the third comparison group are displayed in table 2. Psychometric instruments for scoring in several domains were completed by participants in the baseline survey. The study population characteristics between group one and group two were analyzed for differences. Several scales for assessing socio-economic-status (SES) were used to gauge this characteristic. All ten characteristics were statistically comparable with one exception. Only the CES-D score difference between the groups was statistically significant, ANOVA $(p=0.015)$ at baseline. No other characteristic differences were significant.

The generally higher education attainment of the participant group was evidenced in the socio-economic index (SES score). The depression attributes survey (CESD) and life orientation (LOT) scores were more favorable compared to previously reported general populations scale (Scheier, Carver et al. 1994; Poresky and Clark 2000). The Positive Affect and Negative Affect Scale instrument (PANAS) scores were higher than reported mean scores in the population for the positive affect. Previously reported PANAS mean scores were 33.3 (7.2) for a representative U.S. sample (Watson, Clark et al. 1988).

\section{Research Questions}

The main study research questions included; would the induction of positive affect through electronic media (text/SMS, email) increase positive affect in the treatment group compared to a control group? The instrument to assess this change was the PANAS. Another research question framed included; would 
the induction of daily positive affect be an effective means of driving an increase in daily physical activity in participants in the treatment group compared to a control group in a community environment

\section{Hypotheses}

Ho-1: Patients who received positive affect inductions did not increase their daily positive affect scores on a PANAS scale instrument.

Ho-2: Patients who were targeted for physical activity and received positive affect induction did not yield a change in daily physical activity compared to comparable sets of control subjects.

\section{Results}

The PANAS scores were recorded for the two-week study period Monday through Friday. The daily PANAS requested participants to respond for emotions noted within the previous day (24-hours). A PANAS response was also included in the weekly Saturday battery of surveys. A summary of the PANAS scores are illustrated in table 3. A trend-line chart is illustrated in figure 2 for the positive and negative affect scores of the two treatment groups.

The daily PANAS scores during the treatment and neutral message weeks Tuesday through Friday were tested for TG1 and TG2 in a one-way ANOVA. Each test exceeded the statistical significance threshold of $p 0.05$. No significant differences in the PANAS scores were detected. Monday was established as a baseline score since only one message was sent at 10:00 am prior to the PANAS completion at 1:00 pm. All four days recorded a higher PANAS score than Monday during the positive affect treatment week in both groups. The low 
number of cases in the groups and the low power of the study impacted the analysis.

The daily PANAS scores were analyzed as non-parametric data using the Wilcoxon signed ranks test. The PANAS score differences between the same days in the two study weeks scores (Tuesday of the neutral week compared to Tuesday of the treatment week) exceeded the study statistical significance threshold of $p 0.05$ for each comparison.

Weekly PANAS scores were also collected on consecutive Saturdays. The initial PANAS score was part of the baseline data collection. A second PANAS was recorded immediately preceding the start of the study treatment periods. The PANAS scores requesting a 7-day recall of affect at the end of week-one and week-two were used in the data analysis. A summary of the seven-day Saturday PANAS score means are illustrated in table 4. A graph plot of the PANAS scores for each subject in the two treatment groups is displayed in Figure 8. 
Table 6.

Daily PANAS Scores

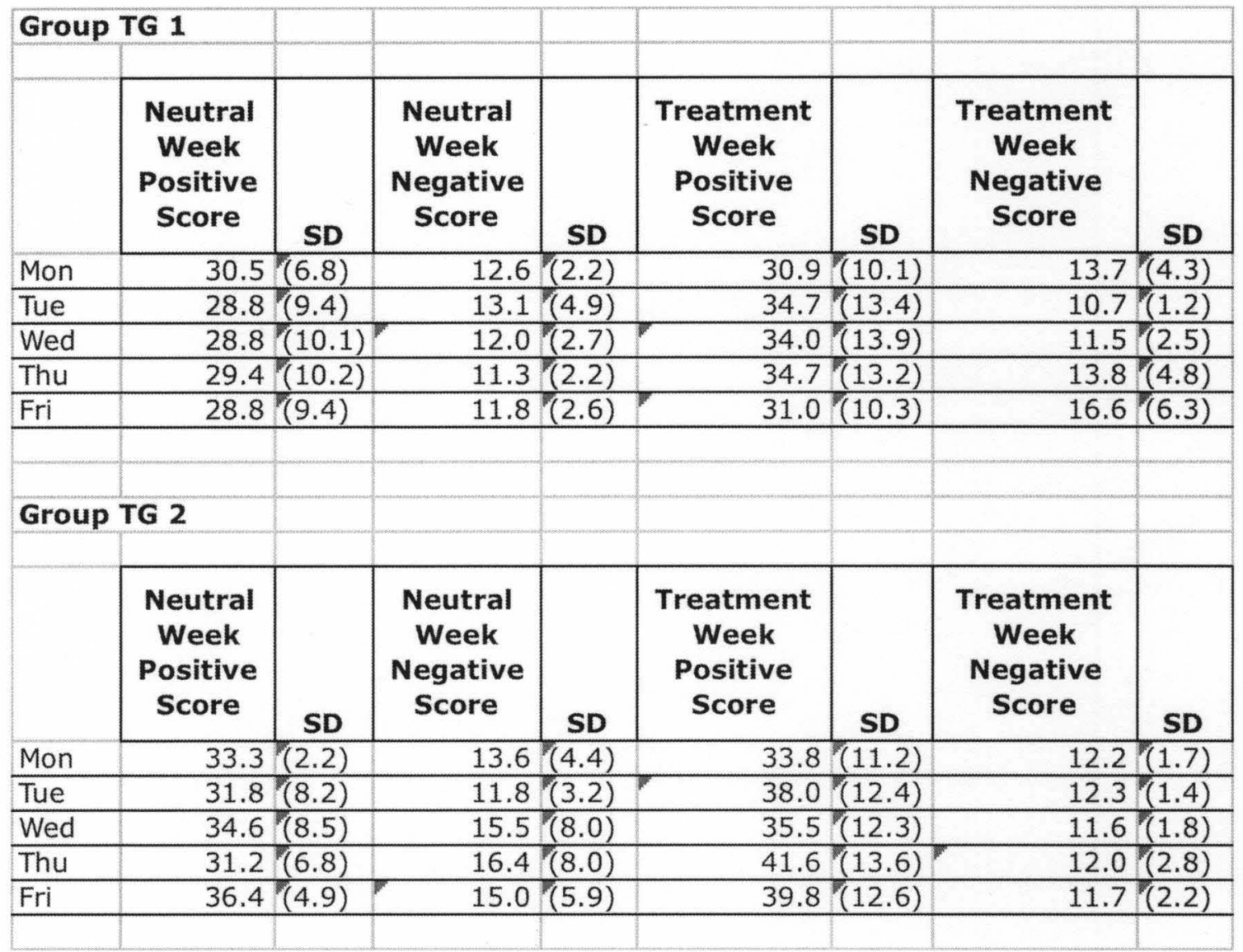

Table 7.

Seven-Day Recall PANAS Scores

\begin{tabular}{|c|c|c|c|c|}
\hline & \multicolumn{3}{|c|}{ Week } & \\
\hline Group & Neutral & SD & Treatment & SD \\
\hline TG1 & 32.6 & $(13.6)$ & 33.4 & $(12.4)$ \\
\hline TG2 & 37.8 & $(10.3)$ & 39.3 & (6.4) \\
\hline & Week 1 & & Week 2 & \\
\hline CG & 38 & (10.8) & 35.5 & (9.6) \\
\hline
\end{tabular}


Figure 8.

Individual PANAS Score Plots for TG 1 and TG2.
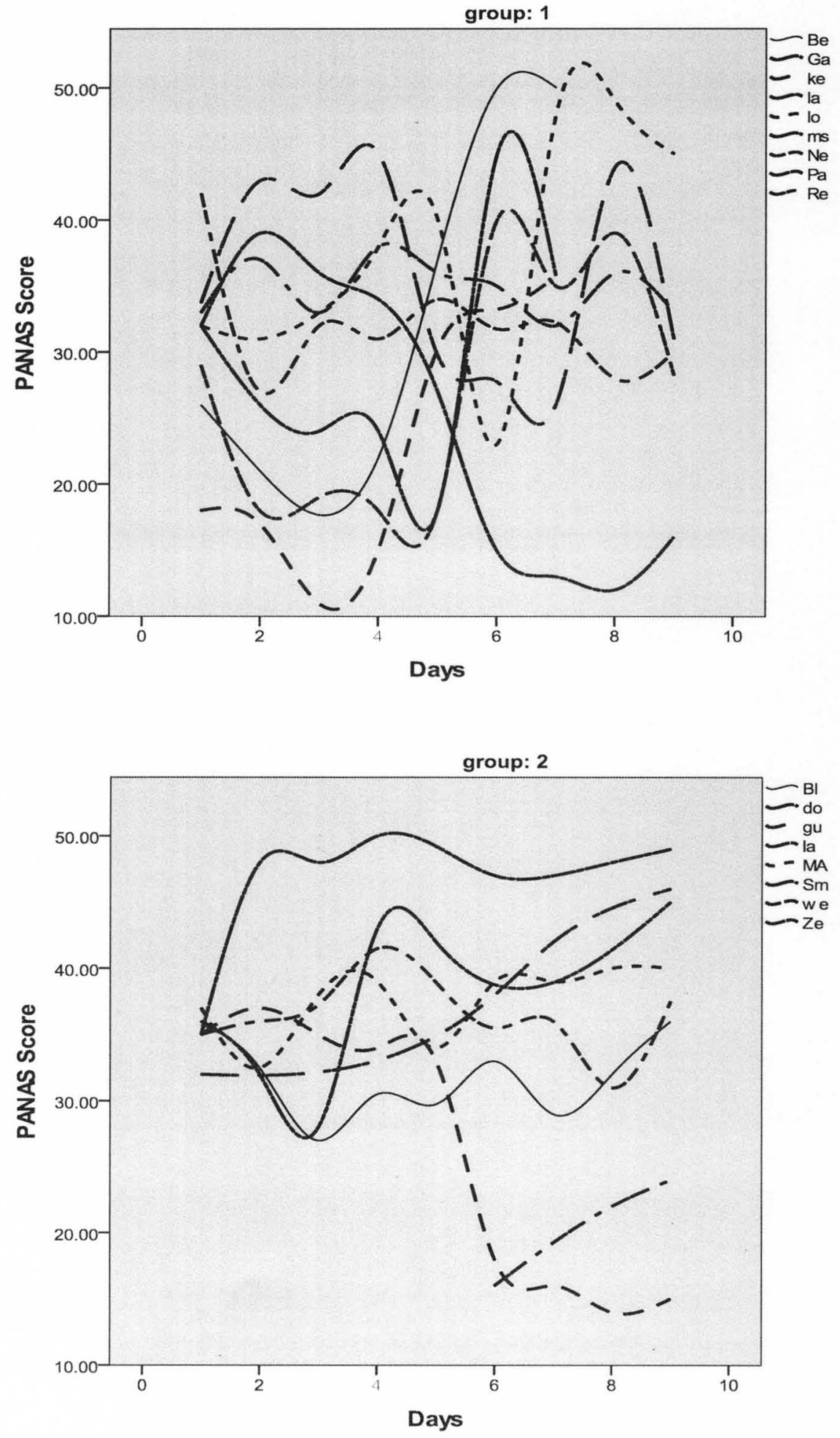
Figure 9.

TG1 and TG2 Daily PANAS Scores

Daily PANAS

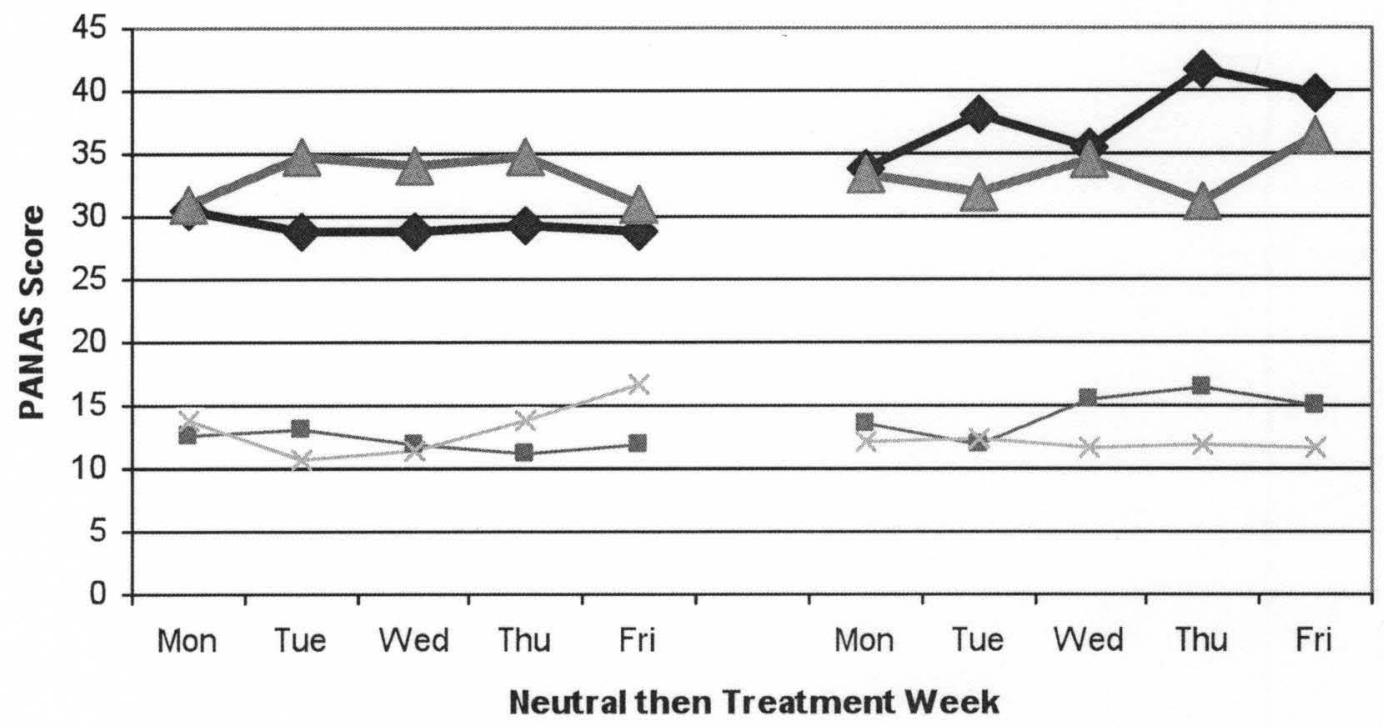

$\longrightarrow$ TG1 pos score Tx - TG1 neg score $=$ TG2 pos score TX - TG2 neg score

Table 8.

Seven-Day Recall SF-36 Role Emotional Sub-Scale

\begin{tabular}{|c|c|c|c|}
\hline & \multicolumn{3}{|c|}{ Week } \\
\hline Group & Neutral & \multicolumn{2}{|c|}{ Treatment } \\
\hline TG 1 & $69.5^{\prime}(25.2)$ & $72.8^{\prime}$ & $(18.8)$ \\
\hline \multirow[t]{2}{*}{ TG 2} & $80.8 \quad(14.5)$ & $85.3^{\prime}$ & $(11.8)$ \\
\hline & Week 1 & \multicolumn{2}{|c|}{ Week 2} \\
\hline CG & $95.0^{\prime}(5.0)$ & $820^{\prime \prime}$ & $\frac{2}{(28)}$ \\
\hline & & & \\
\hline
\end{tabular}


The Saturday PANAS score following the treatment week was compared to the neutral message week PANAS score using a non-parametric MannWhitney test comparing each group. All group results exceeded the study significance threshold and supported the null hypotheses (Ho). Group T1 $(p=0.59)$, Group T2 $(p=0.79)$, Group C $(p=0.46)$.

Weekly SF-36 role emotional scales were collected on consecutive Saturdays. The initial SF-36 score was part of the baseline data collection. The role emotional sub-scale is a component of the total SF-36 score. The collection of the SF-36 responses was a component of the Saturday survey battery. The SF-36 questions requested a seven-day recall of role emotional responses at the end of week-one and week-two for use in the data analysis. A summary of the seven-day Saturday SF-36 role emotional sub-scales are illustrated in table 5.

The Saturday SF-36 role emotional scale or dimension following the treatment week was compared to the neutral message week SF-36 role emotional scale using a non-parametric Mann-Whitney test comparing each group. All groups supported the null hypotheses. Group T1 $(p=0.46)$, Group T2 $(p=0.70)$, Group $C(p=0.18)$.

Pedometer data was available from $22(73 \%)$ study participants. The remaining participants did not return the pedometers or the data indicated less than two days per week of appropriate wearing and data availability. The frequency of missing data for each day in the observation period was greater than $50 \%$. Statistical analysis of the pedometer data was not completed and is not presented in this document. 
The missing data that occurred in the daily PANAS scores and also the weekly PANAS and SF-36 role emotional sub-scale scores were subsequently filled by imputation. Imputation using the nearest two available data point means were calculated using SPSS (SPSS 2008). The statistical analysis previously described was replicated with imputed data. There were no significant changes in any of the statistical functions performed even after imputation. The results of these statistical operations using imputed data are not reported.

\section{Growth Curve Analysis}

The unique characteristics of evaluating data that was effected by treatments over time with multiple observation periods was evaluated using structured equation modeling (SEM) including growth curve analysis (Duncan, Duncan et al. 2006; Preacher, Wichman et al. 2008). The two cohorts of participants in TG1 and TG2, were evaluated for both intra-individual and interindividual differences using growth curve analysis techniques. Each treatment week provided a point zero (Monday) and nine waves of subsequent observations (Tuesday-Friday during the neutral week including Monday of the crossover week, Tuesday-Friday in the treatment week) using the available PANAS scores. Several growth curve models were used to test the hypotheses regarding the increase in PANAS scores over time progressing from neutral messages to the humor messages for TG1 and humor to neutral for TG2. The grand mean fixed effects and the true score variances of random effects would have been expected to differ significantly from zero. All analyses used restricted 
maximum likelihood estimation (REML). Growth curve analysis results are displayed in Table 6.

The intercept of both group and individual PANAS scores differed significantly from the grand mean in all models tested $(p=0.00)$. Thus, the initial PANAS scores as means between groups and the individual starting scores were statistically different. The difference in PANAS score variation increased between days compared to the variation between subjects. The $\chi^{2}$ tests of the variances for the random effects indicated that there was reliable variance among $Y$-intercepts ( $p$ values all $=0.00$ ), but not a statistically significant relationship between days of treatment and PANAS slopes. None of the linear slope analysis were significantly different from zero, $(p>0.05)$ see Table 9.

Several growth curve models were tested with individual covariates inserted in the model to control for each variable. None of these covariates effect was significant in either the influence on the slope or variation reduction. Covariates inserted in this phase of the model testing included; life orientation score, CESD score, body mass index, socio-economic score, confidence score. A growth curve model was tested to fit each subjects PANAS scores, and allow for the possibility that the individual growth curves might differ reliably from the grand mean growth curve. The growth curve models tested evaluate the random effects of individual PANAS scores repeated across days. The variance of each individual PANAS is compared using the $\chi^{2}$ tests. The random measure analysis comparing the variance for daily PANAS scores between the treatment and neutral periods did not find any statistical significance, $p=0.77$. Table 9 
summarizes the covariance parameters for this repeated measures growth curve analysis. The auto regressive models (AR1) indicate that there is a meaningful relationship across measurements within persons in PANAS scores across days TG1, $(p=0.03)$, but not meaningful as a predictor for TTG, $(p=0.09,0.14)$.

Table 9. Growth Curve Outputs

\begin{tabular}{|c|c|c|c|c|c|c|}
\hline \multicolumn{7}{|c|}{ Growth Curve Analysis Fixed Effect } \\
\hline & \multicolumn{3}{|c|}{ TG 1} & \multicolumn{3}{|c|}{ TG 2} \\
\hline & $\begin{array}{c}\text { Mean } \\
\text { Estimate }\end{array}$ & $\begin{array}{c}\text { Standard } \\
\text { Error }\end{array}$ & Sig. & $\begin{array}{c}\text { Mean } \\
\text { Estimate }\end{array}$ & $\begin{array}{c}\text { Standard } \\
\text { Error }\end{array}$ & Sig. \\
\hline Y Intercept & 30.30 & 5.40 & 0.00 & 31.90 & 5.50 & 0.00 \\
\hline Slope & 0.45 & 0.85 & 0.60 & 0.64 & 0.60 & 0.29 \\
\hline \multicolumn{7}{|c|}{ Growth Curve Analysis Random Effect, Repeated Within Subjects } \\
\hline & TG 1 & Sig. & & TG 2 & Sig. & \\
\hline & & & & & & \\
\hline Residual & 0.49 & 0.03 & & 0.50 & 0.09 & \\
\hline$(1,1)$ & 35.50 & 0.69 & & 117.00 & 0.16 & \\
\hline$(2,1)$ & -9.40 & 0.55 & & -7.10 & 0.37 & \\
\hline$(2,2)$ & 2.50 & 0.45 & & 0.50 & 0.64 & \\
\hline AR1 diagonal & 70.06 & 0.03 & & 29.39 & 0.09 & \\
\hline AR1 rho & 0.49 & 0.03 & & 0.46 & 0.14 & \\
\hline
\end{tabular}




\section{Summary}

The results of the daily and weekly PANAS and SF-36 data support the acceptance of the null hypotheses (Ho). That is the difference between groups $\mathrm{T} 1, \mathrm{~T} 2$ and within groups $\mathrm{T} 1, \mathrm{~T} 2$ comparing the positive affect treatment versus neutral message weeks were not statistically significant. The lack of available pedometer data did not allow for meaningful analyses. The a priori statistical analysis plan outlined recommended no physical activity evaluation if the first null hypotheses $(\mathrm{Ho})$ was accepted, as it was. 


\section{CHAPTER V \\ SUMMARY, CONCLUSIONS, RECOMMENDATIONS}

\section{Introduction}

The main study research questions included; would the induction of positive affect through electronic media (text/SMS, email) have increased positive affect in the treatment group compared to a control group? The instrument to assess this change was the PANAS. Another research question framed; was the induction of daily positive affect an effective means of driving an increase in daily physical activity in participants in the treatment group compared to a control group in a community environment

The exploration of improved techniques and intervention designs to influence health behavior is a growing need in the United States. The literature is informative regarding these theories and successful applications, yet the prevalence of lifestyle issues and chronic conditions continue to increase (USDHHS 2000). Lifestyle behaviors such as sedentary habits, poor nutritional intake and excess body fat have been identified as key factors in the development of chronic conditions including heart disease and cancer (Finkelstein, Fiebelkorn et al. 2003; Schroeder 2007). Behavioral change research has evolved in theories and constructs targeting lifestyle changes. There appear to be multiple theories, cognitive and behavioral techniques that have been studied in applied settings (Glanz, Rimer et al. 2002). 
The use of positive affect as a process or adjunctive treatment is an area that may have value in future applications. Positive affect can be induced through an external influence. The topic or issue that is the target of the influence must be viewed as neutral or in a positive perspective for the induction to be effective (Isen and Reeve 2005; Isen 2008). Positive affect induction has not been shown to reverse a negative emotion or feeling. Positive affect appears to increase the positive valence when the issue of interest is viewed as positive or at a minimum neutral (Isen 2005; Isen 2008). This specificity of effect is a key aspect of positive affect. This specificity is not a mood shift that acts as a consistent bias, but a trigger than only effects certain issues (Isen 1999). Emotions and cognitions appear to be bidirectional causes and effects, based upon the trigger event. That is, an emotion can directly influence the cognitive process or the cognitive evaluation of a situation or, conversely, the environment can result in a changed emotional state (Isen 2008).

There are various types of positive affect induction approaches represented in the empirical literature that include imagery; audiotapes, humorous videotapes, video clips, reflection on positive scenarios, autobiographical recall of positive events, fragrance, personal diary of positive events, candy as an unexpected gift, positive word list/association (Lyubomirsky, King et al. 2005; Isen and Erez 2007). The most important factor in consideration of introducing positive affect, is the amount of time or latency, an individual is influenced rather than the intensity of the affect (Lyubomirsky, King 
et al. 2005). Studies previously cited constitute controlled laboratory studies that have not been tested in an applied setting.

The evaluation of positive affect inductions in applied settings across multiple days may be influenced by factors not previously identified. As an example, there is a definite circadian rhythm to levels of positive affect. After rising from sleep, positive affect increases and maintains levels throughout the day, peaks at approximately 8-hours post waking, and then decreases in the evening (Watson, Wiese et al. 1999; Lawton, Conner et al. 2009).

\section{Research Need and Problems}

The research implications and opportunities to explore the impact of positive affect on health care decisions and behavior appears to be numerous. The role of emotions has not been applied to personal decisions regarding medical care and preventive care (Diffenbach, Miller et al. 2008), and is recommended for additional study of nutritional intake (Macht and Simons 2000). It appears that depressed affect supports indecisiveness, yet the impact of this circumstance on health related decisions has not been directly explored (Consedine 2008). The effects of induction of specific emotions such as anger, sadness and fear has not been reported (Macht 2008). It appears that evidence is absent regarding the effect of positive affect on coping behavior and health behaviors (Lyubomirsky, King et al. 2005).

The literature reviewed appears to be missing several facets of positive affect manipulation study. Practical application of induction techniques outside of controlled conditions has not been reported. A multi-week correlation study of 
self-reported emotions has been published (Macht and Simons 2000), but no prospective randomized experimental study of positive affect was found.

\section{Conclusions}

The results of the daily PANAS scores in this study were suggestive of increased positive affect states as a result of the humorous message treatments. The trend-line chart of each group was higher during the treatment week period compared to the neutral message period. The weekly period recall for both the PANAS and SF-36 role emotional scale scores were also suggestive of the same influence although a smaller effect. The comparison of the two main treatment groups to the third comparison group was inconclusive as a result of different neutral week PANAS scores. The comparison group scores increased in the first week and declined during the second week without any treatments. Although the intercepts and variances when measured using growth curve analysis were promising, the slopes of most group comparisons and individuals were within expected ranges of variability.

The negative scale scores during all each observation period supported previously reported performance of independence between the positive and negative values captured through the PANAS. The decline in PANAS scores for the neutral message cohort during the first week suggests that the run-in period and previous exposure to the survey collection process limited a maturation effect. The increase in positive emotion scores through both the PANAS and SF36 instruments appears to limit any probable influence of an artifactual influence of the reporting instrument or any structural bias in the instruments with the 
participants in this study. The crossover study design combined with the increase in positive scores for each appears to have eliminated a historical effect or environmental factor introduction as an influence.

The data analysis used structural equation modeling (SEM), specifically growth curve modeling to account for the multiple data points through time. Growth curve analysis allowed for a comparison of both within group PANAS scores and between group PANAS scores

The increase in the positive PANAS scores during the treatment week was comparable to previous studies of induced positive affect (Isen 2008). The humor treatment appeared to increase positive affect similar to previously reported research data. The seven-day PANAS score increase was supported by the SF-36 role emotional sub-scale increase during the same period. The same direction of change during the same time period recorded through two survey instruments limits the likelihood of a maturation effect or an artifactual influence inherent in the survey instrument.

The lack of evidence and statistically significant findings to support the research questions and disprove the null hypotheses precluded any subsequent analysis of influence on physical activity. The large number of missing data in the pedometer data set limited this analysis. The seven-day physical activity recall data was available but not evaluated as outlined in the statistical evaluation plan documented a priori absent significant positive affect change.

The characteristics of the participant population indicated a more educated and affluent female compared to U.S. population averages. The 
baseline battery of survey data produced a profile of health that was comparable but with less illness and chronic condition influence than the U.S. population. The role emotional health, affect scores, quality of life and functionality scores were comparable but slightly higher than comparative U.S. norms. Differences in characteristics between the three study cohorts were not significant with the sole exception of the CES-D group differences. The low CES-D scores in TG1 were significantly lower than TG2 and the CG.

\section{Discussion}

The results and analysis were challenged due to the small number of respondents to a given survey and the large number of missing data elements. A thorough analysis of the results was not feasible as a result of the small number of cases available through the two-week study. The growth curve analysis appeared to offer a useful statistical model well suited to the applied study of PANAS scores over time. The missing data and smaller number of participants that may be associated with similar future studies are well suited for growth curve analysis. This lack of power limited the statistical tests. The a priori sample size determination for the main study estimated a minimum of 40 participants and potentially 65 participants depending on the induction effect. The erosion of study participants who agreed to informed consent and were consistent participants in study activities was approximately $50 \%$. The pool of volunteers accepted through informed consent will likely require 80 to 130 participants in each study arm as a guideline for follow on studies. The growth curve analysis 
may only require as few as 20 to 30 participants with 10 data points for measurement.

The statistical analysis of the study participants was not conclusive. The small numbers of participants, resulting in under-powered analysis contrasts with suggestive emotional scores that increased during treatment periods were promising but inconclusive. The CES-D scores of group TG1 compared to group TG2 and CG were significantly different. Although randomization appeared to succeed in creating closely matched characteristics in both groups, the PANAS scores during the study were not matched. The PANAS score bias between the groups rendered between group analyses not useful.

The applied nature of the reporting does not allow for respondents to respond at the same time of the day. Late respondents may introduce a confounding factor that is not easily controlled. Consideration of limiting the PANAS collection to the seven-day recall and eliminating the daily scores is practical when reviewing the exploratory study results and the comparison with the main study daily and weekly scores.

The exploratory study was designed to test several aspects of positive affect induction in an applied setting. The induction techniques were explored using qualitative research methods and yielded several insights. The feedback and survey sessions established a practical approach to attempted positive affect inducements.

The use of the accelerometers/pedometers was introduced without difficulty. Missing data on days when participants temporarily lost the device, or 
forget to wear the device were noted. Participants commented on the novelty of the accelerometer and enjoyed the use of the device during the study. The lack of usable pedometer data from most participants was not anticipated. Previous pedometer studies have not reported non-adherence to study protocols to the degree experienced in this study (Merom, Rissel et al. 2007; Clemes and Parker 2009).

\section{Practical Implications}

The distribution of messages through the communication channels was completed without any major obstacles. The email and text distribution of messages were relatively easy to design and deploy. The design and programming required for the operation of the automated interactive phone system would have required a large amount of resources. The time to develop the architecture and program the computer-generated system exceeded 9months. The interactive phone system was not included in the study methodology based on the explorative study findings. The ability to connect with participants at each contact using email or SMS/text messages exceeded $90 \%$.

The timing of the message receipt was not consistent. Participants were sometimes unavailable for phone or email contact and did not always receive messages at the target delivery times. The use of multiple channels for scheduled contact provided flexibility for participants to select the most convenient communication channel.

The novel delivery of the positive affect inductions appears to have merit and was well tolerated by participants. Increased access to multiple 
communication venues may improve the timely delivery of induction messages. The use of positive affect induction with newer technology applications such as public and employer exclusive social media sites combined with peer support and peer humor is a potential concept for exploration. The lost cost of message distribution and easy message replication through multiple communication venues has particular promise for population health applications.

Related results to previous studies

The exploratory study revealed the promise of measuring the influence of positive affect inductions in an applied setting. Although the findings are not conclusive it appears that there is some level of probability that humor induction techniques will influence positive feelings based on the 1.37 point increase in the seven-day PANAS scores. The increase in the PANAS score is consistent with similar controlled laboratory findings (see table 3 , pages $35-36$, chapter 3 ). The use of humor as an intervention technique has the potential to increase positive affect in a similar fashion as previous studies using movies or unexpected gifts. The range and distribution of PANAS scores in the participant groups were comparable to other U.S. population observations (Watson, Clark et al. 1988). This study appears to be the first prospective randomized study of positive affect induction in an applied setting. The use of email and/or SMS text messages through a smartphone as an induction technique was not previously reported in the literature. The use of email and smartphones has been reported for behavior change support in combination with a health educator interaction (Wing, Jeffery et al. 1996; Castro and King 2002; Estabrooks and Smith-Ray 2008; Fjeldsoe, 
Marchall et al. 2009). The attempt to link positive affect and influence on a target health behavior was not previously reported in the literature.

The positive affect induction supported the Broaden and Build Theory through the consistent and higher PANAS scores across time. The influence on PANAS scores through these small emotional manipulations using humor repeated within and across days is explained by the Broaden and Build theoretical construct.

\section{Discussion of Uncontrolled Variables}

The research of positive affect induction and potential influence in an applied setting posed multiple threats to the study and data validity. Positive affect variation between individuals has been previous reported ((Watson, Clark et al. 1988; Isen and Erez 2007). The ranges of daily PANAS scores were large. Larger group size would have been expected to reduce the impact of this variation for future consideration. Positive affect variation within individuals has been documented to follow a circadian rhythm with peak values approximately eight hours post waking (Watson, Wiese et al. 1999). The distribution of the PANAS survey at 1:00 pm to all participants was designed to reduce this factor's variation. The actual time of PANAS completion was not consistent and poses an internal threat to data validity. Historical effect influences through social and environmental issues have the potential to boost or degrade the positive affect responses in the PANAS. As an example, during the treatment weeks the U.S. stock index displayed volatility and included large declines in stock index 
performance. This aspect of historical effect influence on PANAS responses has not been reported.

Randomization appeared to have succeeded based on multiple demographic characteristics. The CES-D scores between groups were observed to be significantly different. Various psychometric characteristics that were not observed had the possibility to influence the PANAS scores of study cohorts, although the literature is silent regarding this type of association or effect.

\section{Recommendations}

\section{Practical Implications}

The exploratory study and anecdotal comments from participants support the use of positive affect induction in behavior change interventions. Although the results of this study were not conclusive but suggestive, there are practical approaches that can be considered. Behavioral change theories typically include techniques to better inform and persuade individuals to attempt a target lifestyle behavior. The incorporation of humor and other positive affect induction into intervention design may have merit. Humor is a sophisticated communication interaction and requires an intimate understanding between the sender and receiver. Research on acceptable humor messages similar to the exploratory study appear prudent in future endeavors. The use of low-cost unexpected gifts may be a lower risk induction technique to explore. A drawback is the single dose impact of an unexpected gift that is harder to deliver longitudinally in multiple doses. When the gift is expected, the impact becomes diminished or 
eliminated. The over-arching implication may be the need to create, deliver and research the use of positive affect inductions in applied settings. Improvement in research design and methodology

Further study of positive affect in applied settings appears worthy of resources and investigation. The exploratory study reviewed here documented promising approaches to applied techniques of affect induction. Many challenges were identified in the scientific exploration process that will require subsequent studies to replicate and conquer.

The small number of participants degraded the statistical power of the analysis, precluding conclusions regarding the impact of the positive affect inductions. This may or may not be influenced by the low power of the analysis. Increased study cohorts with a planned wash-out rate of 50\% appear prudent to overcome this issue.

The daily variations and external confounding factors such as weather, social issues, and news may introduce a history effect bias into PANAS scores. The exclusion of daily PANAS surveys and reliance on the seven-day recall may reduce history effect influences, missing data and participant washout. The seven-day recall displays a more stable level of response reproducibility and may be more suitable for the weekly treatment periods.

The temporal relationship between induction and PANAS score changes and the residual effect of the inductions is not informed from available literature. A longer set of observed PANAS scores will allow for improved analysis and conclusions in the main study. A follow-on study with larger cohorts may still not 
be able to conclude with statistical significance the effect of the inductions, but such a study appears to have merit and advance the scientific knowledge in this field of study 


\section{REFERENCES}

Andrade, E. B. and D. Ariely (2009). "The enduring impact of transient emotions on decision making." Organizational Behavior and Human Decision Processes 109: 9.

Ashby, F. G., A. M. Isen, et al. (1999). "A neuropsychological theory of positive affect and its influence on cognition." Psychological Review 106(3): 22.

Aspinwall, L. G. and S. E. Taylor (1993). "Effects of social comparison direction, threat, and self-esteem on affect, self-evaluation, and expected success." Journal of Personality and Social Psychology 64(5): May 1993.

Ball, K. and D. Crawford (2006). "An investigation of psychological, social and environmental correlates of obesity and weight gain in young women." International Journal of Obesity 30: 9.

Bandura, A. (2001). "Social Cognitive Theory: An Agentic Perspective." Annual Review of Psychology 52: 26.

Barger, S. D., S. M. Burke, et al. (2007). "Do induced moods really influence health perceptions?" Health Psychology 26(1): 11.

Barrett, L. F., J. Gross, et al. (2001). "Knowing what you're feeling and knowing what to do about it: Mapping the relation between emotion differentiation and emotion regulation." Cognition and Emotion 15(6): 12.

Bickmore, T. and T. Giorgino (2006). "Health dialog systems for patients and consumers." Journal of Biomedical Informatics 39: 16.

Blanchard, C. M., M. Fortier, et al. (2007). "Explaining physical activity levels from a self-efficacy perspective: the physical activity counseling trial." Annals of Behavioral Medicine 34(3): 6.

Bless, H., G. Bohner, et al. (1990). "Mood and persuasion: A cognitive response analysis." Personality and Social Psychology Bulletin 16(2): 14.

Bless, H., G. L. Clore, et al. (1996). "Mood and the use of scripts: Does a happy mood really lead to mindlessness?" Journal of Personality and Social Psychology 71(4): 15.

Booth, A. O., C. A. Nowson, et al. (2008). "Evaluation of an interactive, Internetbased weight loss program: a pilot study." Health Education Research 23(3): 11.

Brantley, P. (2008). Weight Loss Maintenance Trial, Protocol. Baton Rouge, Pennington Biomedical Research Center: 74.

Bray, G. A. (2004). Historical Framework. Handbook of Obesity. G. A. Bray and C. Bouchard. New York, Marcel Dekker, Inc.: 1-31.

Busey, J. C. and P. Michael (2008). "Telehealth-opportunities and pitfalls." Journal of the American Dietetic Association 108(8): 6. 
Carnevale, P. J. D. and A. M. Isen (1986). "The influence of positive affect and visual access on the discovery of integrative solutions in bilateral negotiation." Organizational Behavior and Human Decision Processes 37: 14.

Castro, C. M. and A. C. King (2002). "Telephone-assisted counseling for physical activity." Exercise Sport and Science Reviews 30(2): 5.

Chapman, G. B. and E. J. Coups (2006). "Emotions and preventive health behavior: Worry, regret, and influenza vaccination." Health Psychology 25(1): 9.

Chapman, L. S., N. Lesch, et al. (2007). "The role of health and wellness coaching in worksite health promotion." The Art of Health Promotion(July, 2007): 12.

Clemes, S. A. and R. A. A. Parker (2009). "Increasing our understanding of reactivity to pedometers in adults." Medicine \& Science in Sports \& Exercise 41(3): 7.

Consedine, N. S. (2008). Health-Promoting and health-damaging effects of emotions: The view from developmental functionalism. Handbook of Emotions. M. Lewis, J. M. Haviland-Jones and L. F. Barrett. New York, The Guilford Press: 15.

Croyle, R. T. and M. B. Uretsky (1987). "Effects of mood on self-appraisal of health status." Health Psychology 6 (3): 14.

Damasio, A. R., T. J. Grabowski, et al. (2000). "Subcortical and cortical brain activity during the feeling of self-generated emotions." Nature Neuroscience 3(10): 7 .

Delichatsios, H. K., R. H. Friedman, et al. (2001). "Randomized trial of a "talking computer" to improve adults' eating habits." American Journal of Health Promotion 15(4): 10.

Diffenbach, M. A., S. M. Miller, et al. (2008). Emotions and Health Behavior: A self-regulation perspective. Handbook of Emotions. M. Lewis, J. M. HavilandJones and L. F. Barrett. New York, The Guilford Press: 17.

Dishman, R. K., R. W. Motl, et al. (2005). "Self-management strategies mediate self-efficacy and physical activity." American Journal of Preventive Medicine 29(1): 9.

Dovidio, J. F., S. L. Gaertner, et al. (1998). "Intergroup bias: Status, differentiation, and a common in-group identity." Journal of Personality and Social Psychology 75(1): 12.

Dreisbach, G. and T. Goschke (2004). "How positive affect modulates cognitive control: reduced perseveration at the cost of increased distractibility." Journal of Experimental Psychology: Learning, Memory, and Cognition 30(2): 11.

Duncan, T. E., S. C. Duncan, et al. (2006). An introduction to latent variable growth curve modeling. Mahwah, NJ, Lawrence Erlbaum Associates.

Dyer, J. B. and J. G. Crouch (1988). "Effects of running and other activities on moods." Perceptual and Motor Skills 67: 8.

Eakin, E. G., S. P. Lawler, et al. (2007). "Telephone interventions for physical activity and dietary behavior change." American Journal of Preventive Medicine 32(5): 6 . 
Ekman, P. (1992). "An argument for basic emotions." $\underline{\text { Cognition and Emotion }}$ 6(3): 31.

Erber, R. and M. W. Erber (2000). "The self-regulation of moods: Second thoughts on the importance of happiness in everyday life." Psychological Inquiry 11(3): 7 .

Erez, A. and A. M. Isen (2002). "The influence of positive affect on the components of expectancy motivation." Journal of Applied Psychology 87(6): 13.

Estabrooks, P. A., J. A. Shoup, et al. (2009). "Automated telephone counseling for parents of overweight children." American Journal of Preventive Medicine 36(1): 8.

Estabrooks, P. A. and R. L. Smith-Ray (2008). "Piloting a behavioral intervention delivered through interactive voice response telephone messages to promote weight loss in a pre-diabetic population." Patient Education and Counseling 72: 8.

Estrada, C. A., A. M. Isen, et al. (1994). "Positive affect improves creative problem solving and influences reported source of practice satisfaction in physicians." Motivation and Emotion 18(4): 14.

Estrada, C. A., A. M. Isen, et al. (1997). "Positive affect facilitates integration of information and decreases anchoring in reasoning among physicians." Organizational Behavior and Human Decision Processes 72(1): 19.

Finkelstein, E. A., I. C. Fiebelkorn, et al. (2003). "National medical spending attributable to overweight and obesity: How much, and who's paying?" Health Affairs Supplement(W3): 7.

Finucane, M. L., A. Alhakami, et al. (2000). "The affect heuristic in judgments of risks and benefits." Journal of Behavioral Decision Making 13(1): 18.

Fjeldsoe, B. S., A. L. Marchall, et al. (2009). "Behavior change interventions delivered by mobile telephone short-message service." American Journal of Preventive Medicine 36(2): 9.

Forgas, J. P. (1994). "The role of emotion in social judgments: An introductory review and an affect infusion model (AIM)." European Journal of Social Psychology 24: 25.

Forgas, J. P. (1995). "Mood and judgment: The affect infusion model (AIM)." Psychological Bulletin 117(1): 28.

Forgas, J. P. and G. H. Bower (1987). "Mood Effects on Person-Perception Judgements." Journal of Personality and Social Psychology 53(1): 8.

Fredrickson, B. L. (1998). "What good are positive emotions?" Review of General Psychology 2(3): 20.

Fredrickson, B. L. (2001). "The role of positive emotions in positive psychology: The broaden-and-build theory of positive emotions." American Psychologist 56(3): 9 .

Fredrickson, B. L. (2005). Positive Emotions. Handbook of Positive Psychology. C. R. Snyder and S. J. Lopez. New York, Oxford Publishing. 1: 15.

Fredrickson, B. L. and C. Branigan (2005). "Positive emotions broaden the scope of attention and thought-action repertoires." Cognition and Emotion 19(3): 30. 
Fredrickson, B. L. and M. A. Cohn (2008). Positive Emotions. Handbook of Emotions. M. Lewis, J. M. Haviland-Jones and L. F. Barrett. New York, The Guilford Press: 20.

Fredrickson, B. L. and T. Joiner (2002). "Positive emotions trigger upward spirals toward emotional well-being." Psychological Science 13(2): 4.

Friedman, M. and K. D. Brownell (1995). "Psychological correlates of obesity: moving to the next research generation." Psychological Bulletin 117(1): 18.

Futterman, A. D., M. E. Kemeny, et al. (1994). "Immunological and physiological changes associated with induced positive and negative mood."

Psychosomatic Medicine 56: 13.

Gallo, L. C. and K. A. Matthews (2003). "Understanding the association between socioeconomic status and physical health: do negative emotions play a role?" Psychological Bulletin 129(1): 41.

Glanz, K., B. K. Rimer, et al. (2002). Health behavior and halth education: theory research and practice. San Francisco, California, John Wiley \& Sons.

Goldman, S. L., D. T. Kraemer, et al. (1996). "Beliefs about mood moderate the relationship of stress to illness and symptom reporting." Journal of Psychosomatic Research 41(2): 14.

Gordis, L. (2004). Epidemiology. Baltimore, MD, Elsevier Saunders.

Grundy, S. M., G. Blackburn, et al. (1999). "Roundtble Consensus Statement:

Physical activity in the prevention and treatment of obesity and its comorbidities." Medicine and Science in Sports and Exercise 31(11, Supplement): 7.

Hakn, B. E. and A. M. Isen (1993). "The influence of positive affect on variety seeking among safe, enjoyable products." Journal of Consumer Research 20 : 14.

Harvey-Berino, J., S. J. Pintauro, et al. (2002). "The feasibility of using Internet support for the maintenance of weight loss." Behavior change 26(1): 14.

Haynes, R. B., D. L. Sackett, et al. (2006). Clinical Epidemiology. New York, Lippincott Williams and Wilkins.

Hermalin, B. E. and A. M. Isen (2008). "A model of the effect of affect on economic decision making." Quantitative Market Economics 6: 24.

Hill, A. J., C. F. L. Weaver, et al. (1991). "Food craving, dietary restraint and mood." Appetite 17: 11.

Hunter, C. M., A. L. Peterson, et al. (2008). "Weight management using the Internet." American Journal of Preventive Medicine 34(2): 8.

Huntsinger, J. R. and C. T. Smith (2009). "First thought, best thought: positive mood maintains and negative mood degrades implicit-explicit attitude correspondence." Personality and Social Psychology Bulletin 35(2): 11. Isen, A. M. (1998). Positive affect, cognitive flexibility, and self-control: 18. Isen, A. M. (1999). Positive Affect. Handbook of cognition and emotion. T.

Dalgleish and M. Power. West Sussex, John Wiley \& Sons: 19.

Isen, A. M. (2000). "Some perspectives on positive affect and self-regulation." Psychological Inquiry 11(3): 4. 
Isen, A. M. (2001). "An influence of positive affect on decision making in complex situations: Theoretical issues with practical implications." Journal of Consumer Psychology 11(2): 11.

Isen, A. M. (2005). A role for neuropsychology in understanding the facilitating influence of positive affect on social behavior and cognitive processes. Handbook of Positive Psychology. C. R. Snyder and S. J. Lopez. New York, Oxford Publishing. 1: 12.

Isen, A. M. (2008). Some ways in which positive affect influences decision making and problem solving. Handbook of Emotions. M. Lewis, J. M. Haviland-Jones and L. F. Barrett. New York, The Guilford Press: 36.

Isen, A. M., K. A. Daubman, et al. (1987). "Positive affect facilitates creative problem solving." Journal of Personality and Social Psychology 52(6): 10.

Isen, A. M. and A. Erez (2007). Some measurement issues in the study of affect. Oxford Handbook of Methods in Positive Psychology. A. D. Ong and M. H. M. v. Dulmen, Oxford University Press.

Isen, A. M. and P. F. Levin (1972). "Effect of feeling good on helping: Cookies and kindness." Journal of Personality and Social Psychology 21(3): 5.

Isen, A. M., T. E. Nygren, et al. (1988). "Influence of positive affect on the subjective utility of gains and losses: It is just not worth the risk." Journal of Personality and Social Psychology 55(5): 8.

Isen, A. M. and J. Reeve (2005). "The influence of positive affect on intrinsic and extrinsic motivation: Facilitating enjoyment of play, responsible work behavior; and self-control." Motivation and Emotion 29(4): 29.

Isen, A. M., T. E. Shalker, et al. (1978). "Affect, accessibility of material in memory, and behavior: A cognitive loop?" Journal of Personality and Social Psychology 36(1): 13.

Izard, C. E. (2009). "Emotion theory and research: highlights, unanswered questions and emerging issues." Annual Review of Psychology 60: 26.

Kahn, B. E. and A. M. Isen (1993). "The influence of positive affect on variety seeking among safe, enjoyable products." Journal of Consumer Research 20: 14.

Kelsey, K. S., B. M. DeVellis, et al. (2006). "Positive affect, exercise and selfreported health in blue-collar women." American Journal of Health Behavior $30(2): 9$.

Keltner, D. and J. J. Gross (1999). "Functional accounts of emotions." Cognition and Emotion 13(5): 14.

Kuiper, N. A. and N. McHale (2009). "Humor styles as mediators between selfevaluative standards and psychological well-being." The Journal of Psychology 143(4): 18.

LaVeist, T. A. (2005). "Disentangling race and socioeconomic status: a key to understanding health inequalities." Journal of Urban Health 82(2, suppl 3): 8.

LaVeist, T. A., R. J. Thorpe, et al. (2007). "Overcoming confounding of race with socio-economic status and segregation to explore race disparities in smoking." Addiction 102(Suppl. 2): 6. 
Lawton, R., M. Conner, et al. (2009). "Desire or reason: predicting health behaviors from affective and cognitive attitudes." Health Psychology 28(1): 10.

LeDoux, J. (1996). The Emotional Brain. New York, Simon \& Schuster

Lillie-Blanton, M. and T. Laveist (1996). "Race/ethnicity, the social environment and health." Social Science Medicine 43(1): 9.

Lyubomirsky, S., L. King, et al. (2005). "The benefits of frequent positive affect: Does happiness lead to success?" Psychological Bulletin 131(6): 52.

Macdiarmid, J. I. and M. M. Hetherington (1995). "Mood modulation by food: an exploration of affect and cravings in chocolate addicts." British Journal of Clinical Psychology 34: 10.

Macht, M. (1999). "Characteristics of eating in anger, fear, sadness and joy." Appetite 33: 11.

Macht, M. (2008). "How emotions affect eating: A five-way model." Appetite 50: 12.

Macht, M. and G. Simons (2000). "Emotions and eating everyday life." Appetite 35: 7.

Maio, G. R., G. G. Haddock, et al. (2007). "Social psychological factors in tackling obesity." Obesity Reviews 8(Supplement 1): 3.

Martin, L. L., D. W. Ward, et al. (1993). "Mood as input: People have to interpret the motivational implications of their moods." Journal of Personality and Social Psychology 64(3): 10.

McCaul, K. D. and A. B. Mullens (2003). Affect, thought, and self-protective health behavior: the case of worry and cancer screening. Social Psychological Foundations of Health and Illness. J. Suls and K. A. Wallston. Malden, Mass., Blackwell Publishing: 33.

Mellers, B. A. and A. P. McGraw (2001). "Anticipated emotions as guides to choice." Current Directions in Psychological Science 10(6): 5.

Merom, D., C. Rissel, et al. (2007). "Promoting walking with pedometers in the community." American Journal of Preventive Medicine 32(4): 7.

Moskowitz, J. T., E. S. Epel, et al. (2008). "Positive affect uniquely predicts lower risk of mortality in people with diabetes." Health Psychology 27(3S): 10.

Nam, C. B. and M. G. Powers (1983). The Socioeconomic Approach to Status Measurement. Houston, Cap and Gown Press, Inc.

Newman, V. A., S. W. Flatt, et al. (2008). "Telephone counseling promotes dietary change in healthy adults: results of a pilot trial." Journal of the American Dietetic Association 108: 5.

Peters, E., D. Vastfjall, et al. (2006). "Affect and decision making: A "Hot" topic." Journal of Behavioral Decision Making 19: 7.

Poresky, R. H. and K. Clark (2000). "Longitudinal characteristics of the center for epidemiologic studies-depression scale." Psychological Reports 86: 8.

Preacher, K. J., A. L. Wichman, et al. (2008). Latent Growth Curve Modeling. Thousand Oaks, Sage Publications.

Pressman, S. D. and S. Cohen (2005). "Does positive affect influence health?" Psychological Bulletin 131(6): 47. 
Prochaska, J. O., W. F. Velicer, et al. (1994). "Stages of change and decisional balance for 12 problem behaviors." Health Psychology 13(1): 8.

Radloff, L. S. (1977). "The CES-D scale: a self-report depression scale for research in the general population." Applied Psychological Measurement 1(3): 17.

Reiss, A. J. (1961). Occupations and Social Status, The Free Press of Glencoe, Inc.

Richman, L. S., L. Kubzansky, et al. (2005). "Positive emotion and health: going beyond the negative." Health Psychology 24(4): 8.

Roehm, H. A. and M. L. Roehm (2005). "Revisiting the effect of positive mood on variety seeking." Journal of Consumer Research 32: 7.

Rosenberg, E. L. (1998). "Levels of analysis and the organization of affect." Review of General Psychology 2(3): 24.

Russell, J. A. and J. M. Carroll (1999). "On the bipolarity of positive and negative affect." Psychological Bulletin 125(1): 28.

Ryan, R. M. and C. Frederick (1997). "On energy, personality, and health: subjective vitality as a dynamic reflection of well-being." Journal of Personality and Social Psychology 65(3): 36.

Ryff, C. D. and B. Singer (1998). "The contours of positive human health." Psychological Inquiry 9(1): 29.

Sallis, J. F., W. Haskell, et al. (1985). "Physical activity assessment methodology in the Five-City Project." American Journal of Epidemiology 121: 16.

Salovey, P. and D. Birnbaum (1989). "Influence of mood on health-relevant cognitions." Journal of Personality and Social Psychology 57(3): 13.

Salovey, P., J. B. Detweiler, et al. (2001). Affect and health-relevant cognition. Affect and Social Cognition. J. P. Forgas. Mahwah NJ, Lawrence Erlbaun Associates: 20.

Scheier, M. F., C. S. Carver, et al. (1994). "Distinguishing optimism from neuroticism (and trait anxiety, self-mastery, and self-esteem): a reevaluation of the life orientation test." Journal of Personality and Social Psychology 67(6): 16.

Schoenfeld, D. A. (1995, October 24, 1995). "Statistical considerations for clinical trials and acientific experiments." Retrieved December 15, 2009, 2009.

Schroeder, S. (2007). "We can do better - improving the health of the american people." New England Journal of Medicine 357: 8.

Schwarzer, R. (2008). "Modeling health behavior change: how to predict and modify the adoption and maintenance of health behaviors." Applied Psychology 57(1): 30.

Schwarzer, R. and A. Luszczynska (2008). "How to overcome healthcompromising behaviors." European Psychologist 13(2): 11.

Seligman, M. E. P., T. A. Steen, et al. (2005). "Positive psychology progress: Empirical validation of intrventions." American Psychologist 60(5): 11.

Simon, G. E., E. J. Ludman, et al. (2008). "Association between obestiy and depression in middle-aged women." General Hospital Psychiatry 30: 8.

Slovic, P., E. Peters, et al. (2005). "Affect, risk, and decision making." Health Psychology 24(4 Supplement): 6 . 
Smith, P. M., R. Cameron, et al. (2004). "Telephone Counseling for populationbased smoking cessation." American Journal of Health Behavior 28(3): 11.

Smith, S. M. and D. R. Shaffer (1991). "The effects of good moods on systematic processing: Willing but not able, or able but not willing?" Motivation and Emotion 15(4): 36.

SPSS (2008). SPSS 16 Guide to Data Analysis. M. J. Norusis. Upper Saddle River NJ, Prentice Hall.

Stunkard, A. J., Faith Myles S., Allison Kelly C. (2003). "Depression and Obesity." Society of Biological Psychiatry 54: 8.

Tate, D. F., E. H. Jackvony, et al. (2006). "A randomized trial comparing human e-mail counseling, computer-automated tailored counseling, and no counseling in an internet weight loss program." Archives of Internal Medicine 166(August, 2006): 6.

Tate, D. F., R. R. Wing, et al. (2001). "Using Internet technology to deliver a behavioral weight loss program." Journal of the American Medical Association 285: 6.

Teasdale, J. D. and S. J. Fogarty (1979). "Differential effects of induced mood on retrieval of pleasant and unpleasant events from episodic memory." Journal of Abnormal Psychology 88(3): 10.

Teasdale, J. D. and M. L. Russell (1983). "Differential effects of induced mood on the recall of positive, negative and neutral words." British Journal of Clinical Psychology 22: 8.

Thomas, S., J. Reading, et al. (1992). "Revision of the Physical Activity Readiness Questionnaire (PAR-Q)." Canadian Journal of Sport Sciences $17(4): 8$.

Tucker, J. S., M. Orlando, et al. (2006). "Affective and behavioral responses to health-related social control." Health Psychology 25(6): 8.

Tucker, L. A., A. J. Cook, et al. (2008). "Telephone-based diet and exercise coaching and a weight-loss supplement result in weight and fat loss in 120 men and women." American Journal of Health Promotion 23(2): 9.

USDHHS, U. S. D. o. H. a. H. S. (1996). Physical Activity and Health: A Report of the Surgeon General. P. USDHHS, Office of the Surgeon General. Atlanta, GA, National Center for Chronic Disease Prevention and Health Promotion.

USDHHS, U. S. D. o. H. a. H. S. (2000). Healthy People 2010. P. H. S. U. S. Department of Health and Humana Services, Office of the Surgeon General. Washington DC, U.S. Government Printing Office. 2: 60.

Vanwormer, J. J., J. L. Boucher, et al. (2006). "Telephone-based counseling improves dietary fat, fruit, and vegetable consumption: a best-evidence synthesis." Journal of the American Dietetic Association 106(9): 11.

Wardle, J., A. M. Haase, et al. (2004). "Gender differences in food choice: the contribution of health beliefs and dieting." Annals of Behavioral Medicine 27(2): 10.

Watson, D. (2005). Positive affectivity, the disposition to experience pleasurable emotional states. Handbook of Positive Psychology. C. R. Snyder and S. J. Lopez. New York, Oxford: 14. 
Watson, D., L. A. Clark, et al. (1988). "Development and validation of brief measures of positive and negative affect: the PANAS scales." Journal of Personality and Social Psychology 54(6): 8.

Watson, D., D. Wiese, et al. (1999). "The two general activation systems of affect: Structural findings, evolutionary considerations, and psychobiological evidence." Journal of Personality and Social Psychology 76(5): 19.

Wing, R. R. and C. G. Greeno (1994). "Behavioral and psychosocial aspects of obesity and its treatment." Bailliere's Clinical Endocrinology and Metabolism 8(3): 14.

Wing, R. R., R. W. Jeffery, et al. (1996). "Effect of frequent phone contacts and optional food provision on maintenance of weight loss." Annals of Behavioral Medicine 18(3): 5.

Zajonc, R. B. (1980). "Feeling and thinking, preferences need no inferences." American Psychologist 35(2): 25.

Zajonc, R. B. (1984). "On the primacy of affect." American Psychologist 39(2): 7. 


\section{Appendix 1}

\section{Participant Recruitment Flyer}

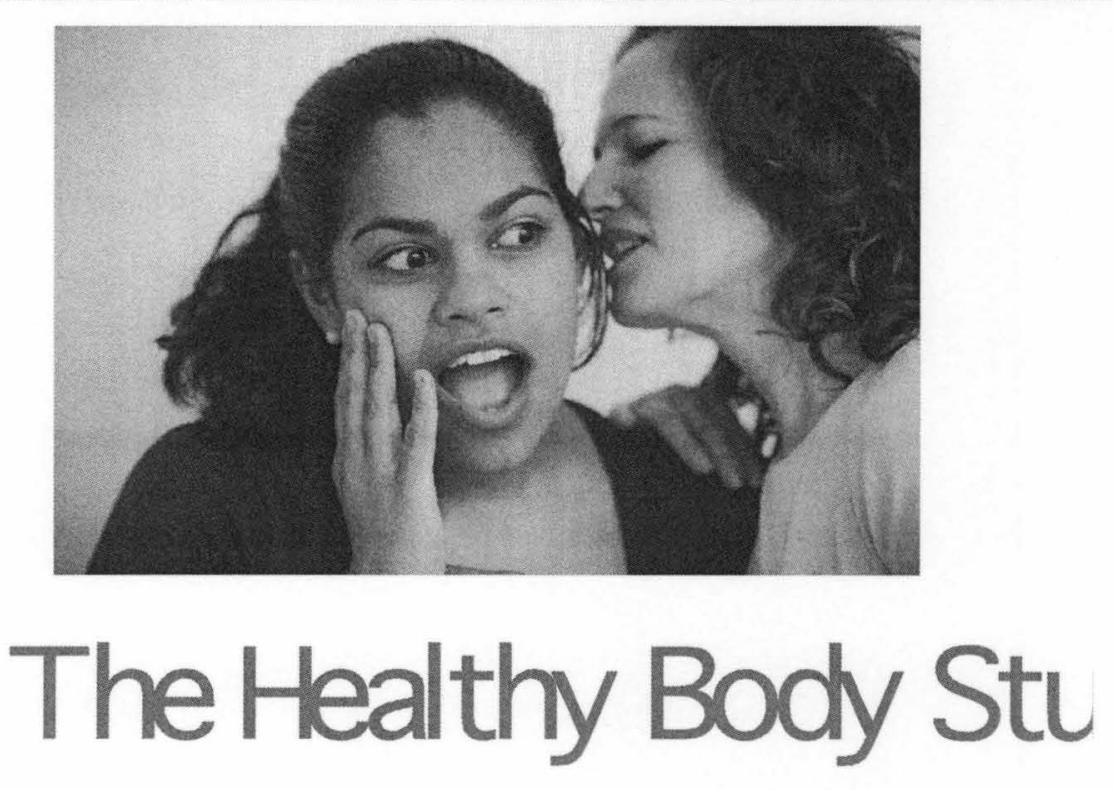

Are you a woman 21-45 years of age? If yes, you may be eligible to participate in a health behavior and emotion study through the UofL.

This study will require a 6-week commitment that includes health surveys, pedometers and phone contact. You will be asked to complete forms, participate in a brief interview and respond to daily phone calls. Surveys will occur at the end of the program also. The study is voluntary without compensation. Group orientation will be held at the UofL Public Health and Health Promotion Dept. 485 Gray Street, Louisville KY.

Study Participants will receive

- Free health information

- Pedometers for use during the study

- Daily phone calls about health behaviors

Please contact Philip Smeltzer, Student Investigator, (502) 744-9293 or philip.smeltzer@louisville.edu to volunteer. Richard Wilson, Principal Investigator, 485 Gray St. Louisville KY 40202 


\section{Appendix 2}

Humor Treatment Message Samples

Chocolate is a vegetable. How, you ask? Chocolate is derived from cacao beans.

Bean = vegetable. Sugar is derived from either sugar CANE or sugar BEETS.

Both are plants, which places them in the vegetable category. Thus, chocolate is a vegetable.

My mother's menu consisted of two choices, take it, or leave it.

\section{Buddy Hackett}

A bagel is a doughnut with the skin removed.

George Rosenbaum

Self delusion is pulling in your stomach when you step on the scale.

Paul Sweeney

The second day of a diet is always easier than the first. By the second day you're off it.

Jackie Gleason

Those who flee temptation generally leave a forwarding address.

Lane Olinghouse.

I always wanted to be somebody. I see now that I should have been more specific.

Lily Tomlin 
I keep trying to lose weight, but it keeps finding me.

Vegetarian - that's an old Indian word meaning lousy hunter.

Andy Rooney

If at first you don't succeed, you're just about average.

Bill Cosby

The best way to get husbands to do something is to suggest that perhaps they are too old to do it.

Shirley Maclaine

Bathroom scales: equipment which only seems to work correctly when one hold on to towel rail, stands on one foot and leans hard to the left.

Men like to barbecue. Men will cook if danger is involved.

Rita Rudner

A diet is when you have to go to some length to change your width.

Too many Americans go in for weight lifting with the wrong equipment, a knife and a fork.

I found there was only one way to look thin, hang out with fat people.

Rodney Dangerfield

My idea of a balanced diet is a quarter pounder in each hand.

George Foreman 


\section{Appendix 3}

\section{Neutral Treatment, General Health Message Samples}

\section{Stress and Exercise}

Has the recession forced you to cut back on things, including your regular workout? Psychologists have found that during a recession, people are more likely to experience a loss of control that can lead to excessive eating and drinking. Whether it is in your local fitness center or at home, it is important to schedule time each day to exercise. It is also one of the least expensive ways to reduce stress and improve your overall health.

\section{Hydration}

How much water should you drink each day? Your water needs depend on your health, where you live and how active you are. But in general, most adults need about nine to 13 servings ( 8 ounces each) of fluids each day. Most fluids, even chicken soup and orange juice, count as part of your fluid intake. If a glass of water sounds boring, spruce it up. Garnish your glass with a mint sprig and a slice of lime, lemon, orange, pineapple, kiwi, star fruit or even a few fresh berries. 


\section{Skipping meals}

Do you frequently skip meals? Do you always deny yourself the foods that you most enjoy? For some people, these behaviors actually interfere with maintaining a healthy weight. Don't hesitate to consult a health educator or dietician. She or he can help you develop a balanced approach to eating the foods you love.

\section{Kids and Exercise}

Consider using family time to get more physical activity. While out for a walk or jog, have your kids ride their bikes alongside you. While walking around a school track, your kids can play on the infield. Being with your children can go hand-in-hand with keeping fit. An added benefit is that they see that you value physical activity.

\section{Nuts}

Nuts are a good source of protein, fiber and beneficial fats. Almonds, walnuts, peanuts and peanut butter are rich sources of heart-healthy unsaturated fats, which lower LDL (bad) cholesterol when substituted for saturated fat. But DO stay informed of any product recalls by visiting www.fda.gov. DO NOT eat products that have been recalled. It's also important to remember that nuts are a calorie-dense food, so keep portion size in mind. 


\section{Appendix 4}

\section{Positive and Negative Affect Scale Survey Instrument}

\section{Feelings and Emotions}

The following scale consists of a number of words that describe different feelings and emotions. Read each item and then mark the appropriate answer in the space next to that word. Indicate to what extent over the past week you have felt this way.

Use the following scale to record your answers.

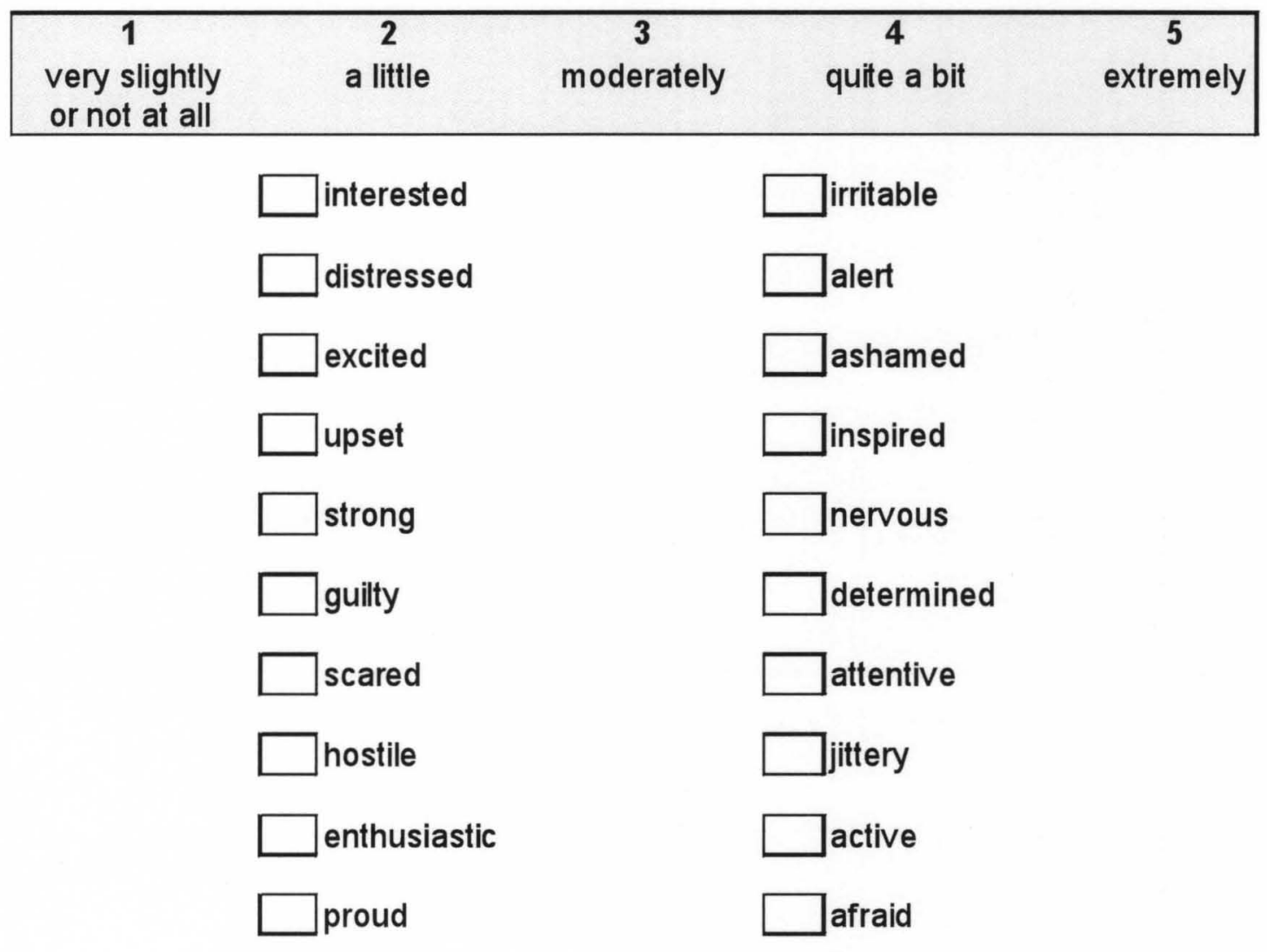




\section{Appendix 5}

\section{Pilot Study Summary}

\section{Introduction}

An exploratory study was planned to validate positive affect induction methods. A qualitative study of induction techniques followed by a limited quantitative analysis was conducted. This preliminary study was designed to empirically study the ability to induce positive affect in a participant group. The study is intended to increase the accuracy of the induction methodology and serve as a validation of the screening procedures and other methods. Participant tolerance for the daily contact and pedometer use will also be validated. This will also serve as a training opportunity for examiners.

Qualitative studies with for 30 participants were planned without randomization. Audio and printed media using phone, email or postal mail as inductions methods were scheduled for implementation in the exploratory study. Positive affect induction methods to be evaluated will include word lists, humorous vignettes, and social support of peers, autobiographical recall, unexpected gifts, positive feedback and affirmations.

The participants were asked to complete all forms and screening instruments. Three group meetings of were held during the qualitative study to evaluate the various induction methods and obtain satisfaction feedback and scoring on the effectiveness of each method to induce positive affect. The humor 
vignettes and jokes were obtained through an Internet search and www. Jokes.com for health related humor.

Each individual in the pilot group received a pedometer and instruction on proper use. Pilot study participants received the positive affect inductions daily over a five-day period to evaluate tolerance and validate induction system operations.

Participants were asked to wear their pedometers/accelerometers for the two-week period after distribution. The message distribution process and interactions were planned to mimic the methodology and procedure planned for the main study. A single humor message/joke was distributed to participants at four times per day over a five-day period. Messages were delivered through email or text (SMS) at 10:00 am, 2:00 pm, 4:00 pm and 8:00 pm. Respondents provided the preferred email addresses and/or phone numbers for humor text message delivery.

The influence on positive affect was measured through completion of the self-reported Positive and Negative Affect Survey (PANAS). The PANAS was accessed through an Internet survey application. A link for the daily completion of the PANAS was distributed at 1:00 on each of the five-day treatment periods. A seven-day recall PANAS was completed on Saturdays by participants at the baseline intake, prior to the treatment period and at the end of the induction treatment period. A set of daily comparison period PANAS scores were collected two weeks after the treatment week. 
The participants returned the accelerometer to collection locations following the study period.

Modifications to the study methodology will be completed based on the qualitative data analysis and conclusions drawn from participants. The conclusions and recommendations obtained through the planned exploratory study will be considered for modification of the procedures in the main study.

\section{Participants}

Recruiting and Selection

A group of 38 participants were recruited over an eight-month period. The planned methodology was to randomly select 50 volunteers from a pool of more than 300 individuals. The first six-months of recruiting efforts were primarily unsuccessful. Medical patients in physician clinic offices were offered the opportunity to volunteer for the study. This effort produced four volunteers in sixmonths. E-mail and newsletter distributions to students, staff and faculty of a university produced 5 volunteers. Flyers posted in high traffic locations of an urban employer were unsuccessful. Four health care employers with large employee populations were requested to allow for direct employee contact for recruitment. Employer permission was denied in each case. Individual employee contacts in two large employers obtained support and access to volunteers. 29 volunteers were obtained through these individual network recruiting efforts.

Study volunteers were requested to schedule either a 15-minute phone appointment to review the informed consent process, or a face to face informed 
consent counseling session. Some volunteers did not attend scheduled informed consent sessions, resulting in 27 volunteers who agreed to participate and completed the informed consent process.

\section{Demographics}

The demographic profile of the participants is displayed in table 1. The study cohort was generally Caucasian, married, college educated, employed full time.

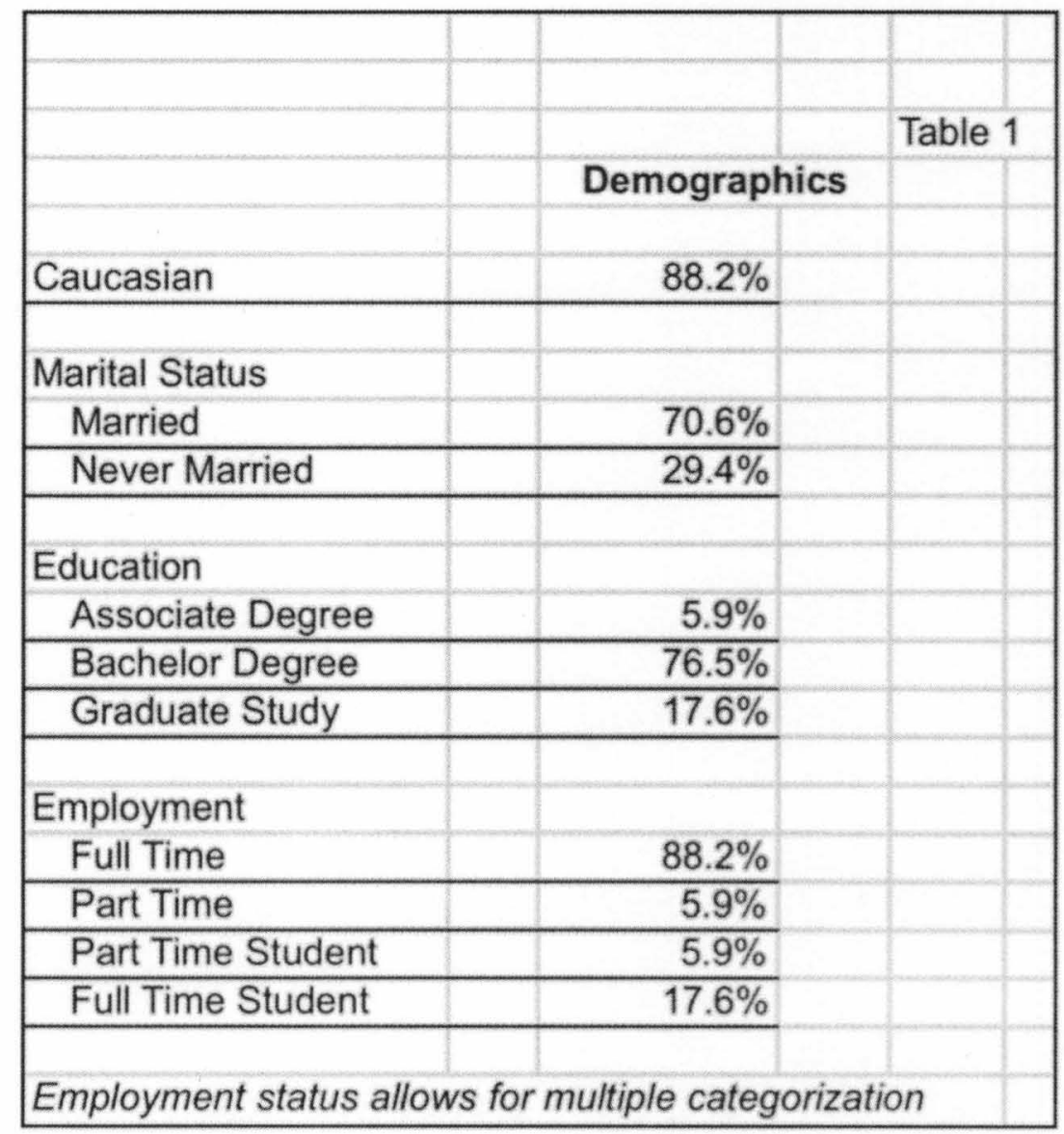

\section{$\underline{\text { Characteristics }}$}

The additional characteristics of the study group are displayed in table 2 . Several scales for assessing socio-economic-status (SES) were used to gauge 
this characteristic. Psycho-metric instruments for scoring in several domains were completed by participants in the baseline survey. The self-efficacy score and confidence to change score were designed through previously validated instruments with a score calculated for statistical analysis convenience.

The generally higher education attainment of the participant group was observed in the educational index, income score and occupational index. A socio-economic index was calculated as an additional characteristic. The CES-D score mean was higher than anticipated and above means informed by the literature in previous studies. The life orientation score (LOT) was similar to previously reported general populations. The higher educated, working female profile of participants may be contributing to the higher characteristic scores.

CES-D screening results indicated two volunteers who were above the common threshold of 19 points. The volunteers who scored 26 and 54 points were encouraged to discuss the results with their personal medical provider and were excluded from the study results. PAR-Q screening revealed one individual who supplied a positive response to the physical limitation question. Subsequent inquiry revealed the issue to be minor and the study participation including daily walking was deemed acceptable. 


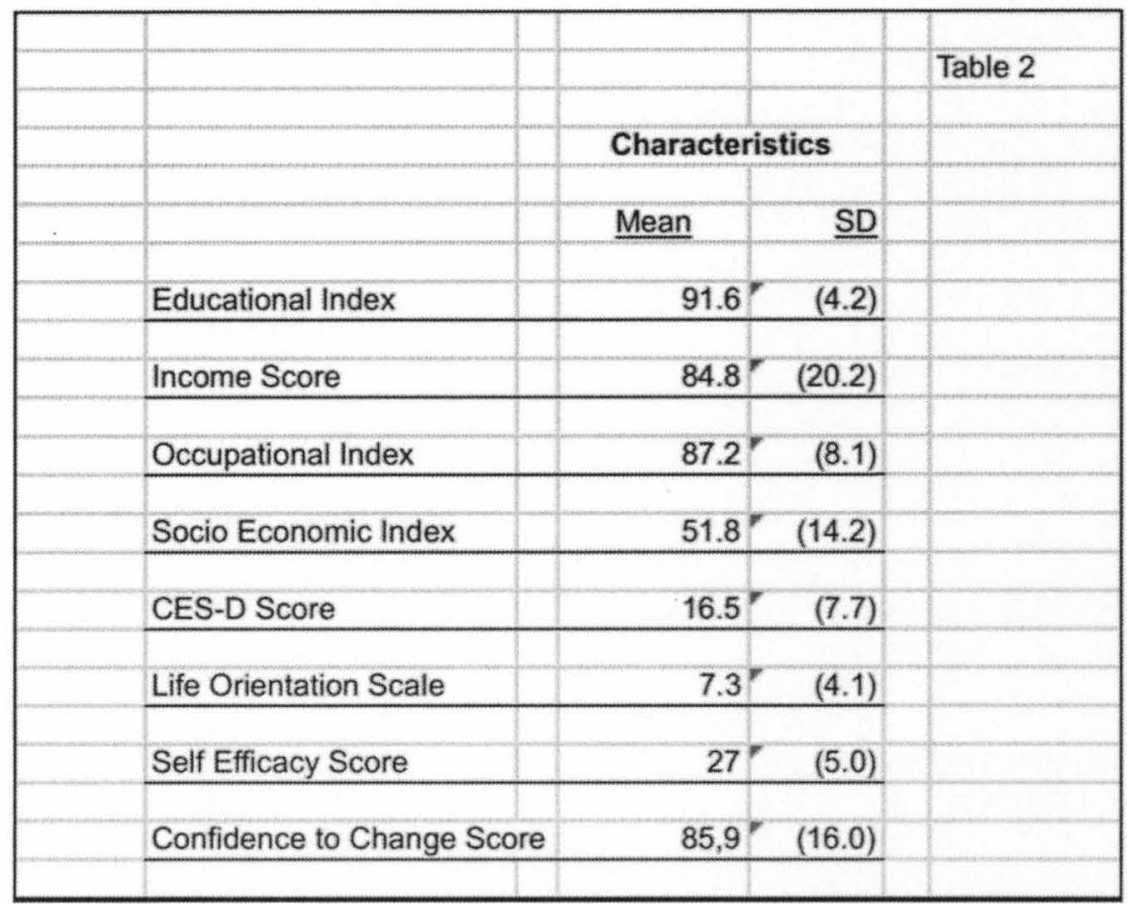

\section{Research Questions}

The exploratory study conclusions and findings are expected to support the tolerance for messages distributed four times per day and a daily PANAS completion.

The induction of positive affect through the daily messages is expected to increase the PANAS scores within individuals during the week of induction.

The main study conclusions and findings are expected to support the successful induction of positive affect. If the findings during this exploratory study conclude that one or all methods are not effective in increasing positive affect, the primary study design may require modification.

The main study research questions are outlined below. 
Can positive affect be induced in an applied setting across multiple days, comparing treatment and control groups?

A secondary research question is, will the induction of positive affect through a variety of electronic media (text/SMS, email, Twitter) increase positive affect in the treatment group compared to a control group? The instrument to assess this change is the PANAS.

Another secondary research question framed becomes, will the induction of daily positive affect be an effective means of driving an increase in daily physical activity in participants in the treatment group compared to a control group in a community environment Hypotheses

Ho-1: Patients who receive positive affect inductions will not increase their daily positive affect scores on a PANAS scale instrument.

Ho-2: Patients who are targeted for physical activity and receive positive affect induction will not yield a change in daily physical activity compared to comparable sets of control subjects.

\section{Results}

$\underline{\text { Qualitative Results }}$

Modifications to the study methodology were completed based on the qualitative data analysis and conclusions drawn from participants. The primary finding was a recommendation to distribute daily messages through email and/or phone text message (SMS). Phone calls with IVR system interface for message delivery was the least preferred delivery method. The protocol was modified, 
eliminating the phone IVR system and adding email and text message delivery capabilities.

Participants attended one feedback session. Three sessions were conducted to capture feedback from all volunteer participants. Participants were exposed to a library of planned telephone communications. The messages were presented on a screen and participants read the messages and immediately rated each potential communication on a scale from one to five through a remote keypad for audience response. The first session was used to narrow potential messages from a library of 278 messages to 90 total messages.

The message rating in the initial session $(n=70$ was analyzed and messages selected were unanimous highest possible ratings. Messages in the bottom $10 \%$ were examined for common traits. This process yielded 15 general health messages, 13 affirmations and 23 humor messages. The second session of study participants rated 30 messages in each category, inclusive of the highest rated messages from the first session. The third session repeated the same 30 messages in each category for rating and feedback. All messages were scored and the highest ratings for 24 messages in each category were selected for inclusion in the main study.

The conclusion of the qualitative research supports the feasibility of multiple daily induction methods. The use of electronic media channels was preferred to phone calls. Daily survey completion through a hyperlink to a survey application within an email message for the daily PANAS survey and weekly surveys was considered acceptable by study participants. 


\section{Quantitative Results}

The baseline and screening battery of surveys was completed by 18 respondents. Each participant received an accelerometer for daily physical activity monitoring after completing the baseline and screening instruments. A follow-up survey seven-days post-baseline was obtained from 7 respondents. A final survey at the conclusion of the treatment period with a seven-day recall on survey responses was distributed and completed by 13 respondents.

The PANAS scores captured for the baseline and final survey requested respondents to recall feeling for the previous seven-days. The daily PANAS requested participants to respond for feelings noted within the previous day (24hours). The PANAS score for the positive induction treatment period, Monday through Friday and the one-week comparison period were collected. A summary of the unadjusted PANAS scores are illustrated in figure 1. A trend-line chart illustrated in figure 2 for the positive affect scores and figure 3 for the negative affect scores.

The weekly PANAS scores were analyzed as non-parametric data using the Wilcoxon signed ranks test. The PANAS score differences between the neutral week and the treatment week were not statistically significant $(p=0.57)$. A summary of the seven-day Saturday PANAS scores are illustrated in table 4.

Three of the four days recorded a higher PANAS score than Monday. The non-parametric Wilcoxon signed ranks test for Thursday compared to Monday $(p=.49)$ was not statistically significant. All other days were found to less 
than statistically significant. The low number of cases between groups was a factor in the analysis.

\begin{tabular}{|c|c|c|c|}
\hline & & & Figure 1 \\
\hline & \multicolumn{2}{|c|}{ PANAS Scores } & \\
\hline & \multicolumn{2}{|c|}{ Positive Scale } & \\
\hline & & & \\
\hline & $\underline{N}$ & Mean & $\underline{\mathrm{SD}}$ \\
\hline Baseline 7 day & $\overline{16}$ & 34.94 & $(8.7)$ \\
\hline Final 7 day & 13 & 36.31 & $(8.9)$ \\
\hline Monday (Baseline) & 14 & 32.36 & (8.2) \\
\hline Tuesday & 19 & 35.00 & (8.5) \\
\hline Wednesday & 10 & 30.90 & $(10.6)$ \\
\hline Thursday & 12 & 33.58 & $(10.2)$ \\
\hline Friday & 11 & 34.82 & $(11.5)$ \\
\hline & & & \\
\hline & PANAS S & & \\
\hline & Negative & & \\
\hline & & & \\
\hline & $\underline{N}$ & Mean & $\underline{\mathrm{SD}}$ \\
\hline Baseline 7 day & 16 & 16.00 & $(6.5)$ \\
\hline Final 7 day & 13 & 14.00 & $(2.6)$ \\
\hline Monday (Baseline) & 14 & 15.71 & $(4.7)$ \\
\hline Tuesday & 19 & 17.21 & $(8.5)$ \\
\hline Wednesday & 10 & 14.80 & $(10.6)$ \\
\hline Thursday & 12 & 13.42 & $(10.2)$ \\
\hline Friday & 11 & 12.55 & $(11.5)$ \\
\hline
\end{tabular}


Figure 2.

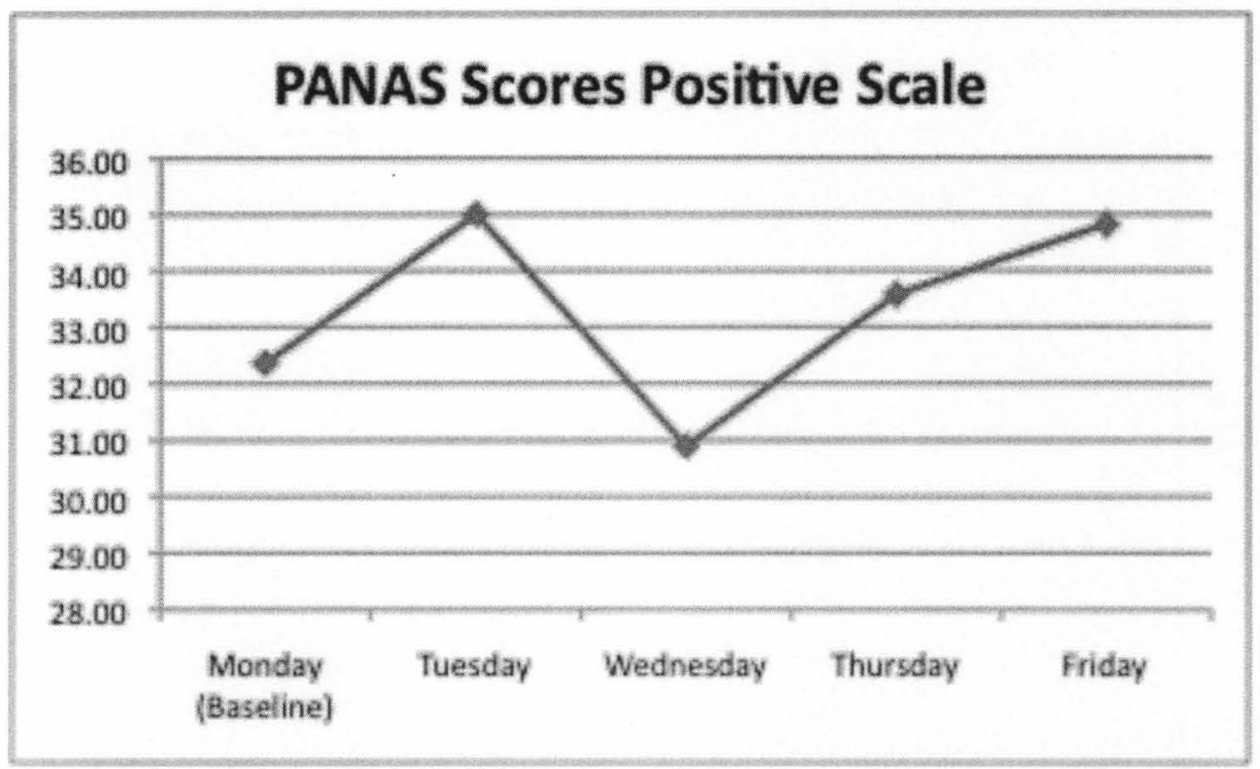

Figure 3 .

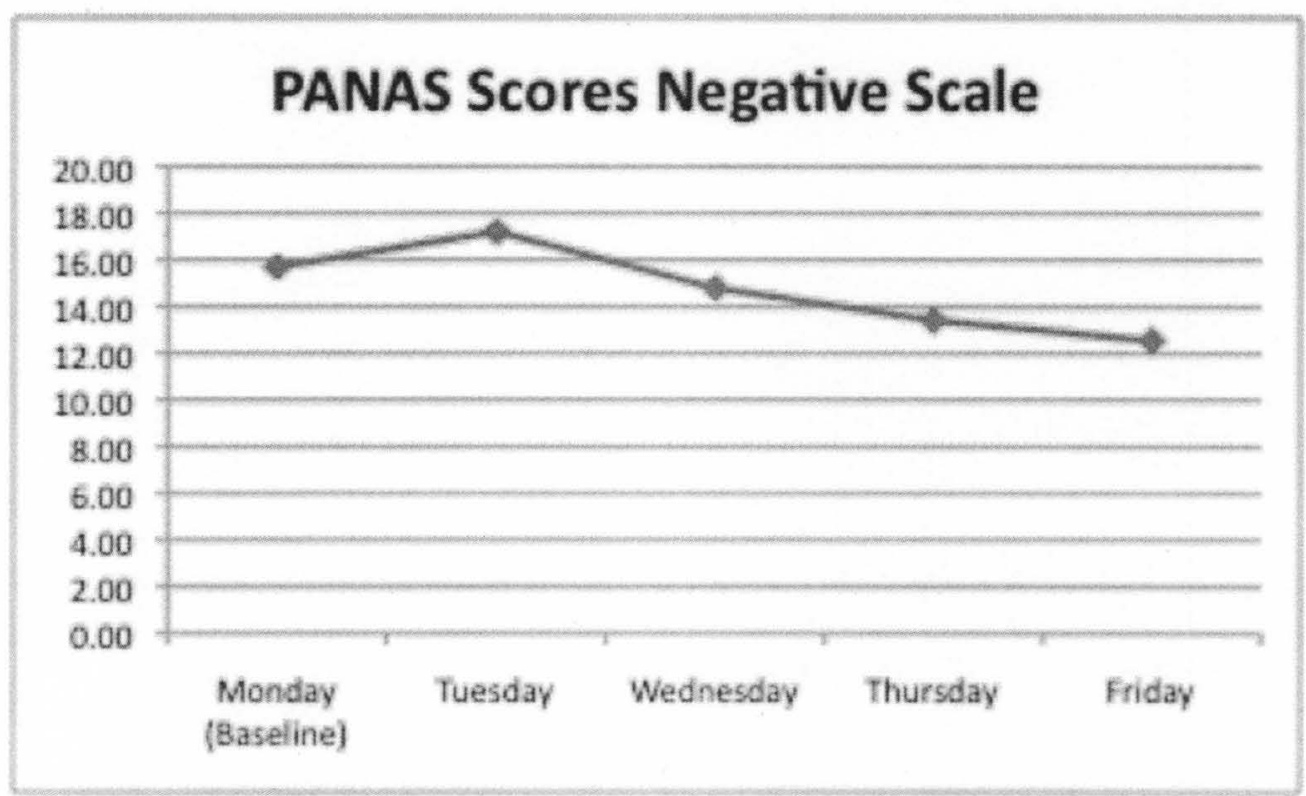


The induction of daily positive affect in participants was not proven to increase the PANAS scores. The low number of participants appears to be a factor in the statistical analysis. The exploratory study supported the null hypothesis, no difference between the treatment period and comparative period within participants was detected. The subsequent analysis of physical activity levels is not reported.

\section{Summary}

The qualitative investigation results of the exploratory study provided insights and practical solutions for use in the main study. The library of messages was distilled into 24 messages in each of three categories. Elaboration on themes and common traits of messages increased the confidence and strength of the selected messages. Practical suggestions to use telephone text messages (SMS) and email will improve the ability to distribute the main study messages and expected increase in the message dosage during the positive affect treatment period. This precludes the requirement for an IVR telephone system during the main study and increases the tolerance for daily contact.

The results and analysis were challenged due to the small number of respondents to a given survey and the large number of missing cases. A thorough analysis of the results was not feasible as a result of the small number of cases available through the two-week study. This lack of power rendered most statistical tests not practical. The sample size determination for the main 
study estimated a minimum of 40 participants and potentially 65 participants depending on the induction effect. The erosion of study participants who agreed to informed consent and were consistent participants in study activities was approximately $50 \%$. The pool of volunteers accepted through informed consent will likely require 80 to 130 participants in each study arm.

The inability to adequately answer the research questions and hypothesis degrades the confidence to implement the main study. The daily PANAS scores may not display adequate specificity. The applied nature of the reporting does not allow for respondents to respond at the same time of the day. Late respondents may introduce a confounding factor that is not easily controlled. Consideration of limiting the PANAS collection to the 7-day recall and eliminating the daily scores is practical when reviewing the exploratory study results.

An unexpected result was the reported negative PANAS scores. Negative affect PANAS scores are collected to monitor unexpected emotional induction or bias. The limited data set precluded any statistically significant conclusions. There was a trend for the negative PANAS scores to decrease during the treatment week. This warrants exploration during the main study. 


\section{EXPLORATORY STUDY SUMMARY, CONCLUSIONS, RECOMMENDATIONS}

\section{Introduction}

The research questions that frame the planned main study and the exploratory study are:

Can positive affect be induced in an applied setting across multiple days, comparing treatment and control groups?

A secondary research question is, will the induction of positive affect through a telephone based interactive voice response system (IVR) increase positive affect in the treatment group compared to a control group? The instrument to assess this change is the PANAS.

Another secondary research question framed becomes, will the induction of daily positive affect be an effective means of driving an increase in daily physical activity in participants in the treatment group compared to a control group in a community environment

The exploration of improved techniques and intervention designs to influence health behavior is a growing need in the United States. The literature is informative regarding these theories and successful applications, yet the prevalence of lifestyle issues and chronic conditions continue to increase [1]. Lifestyle behaviors such as sedentary habits, poor nutritional intake and excess 
body fat have been identified as key factors in the development of chronic conditions including heart disease and cancer $[2,3]$. Behavioral modification research has evolved in theories and constructs targeting lifestyle changes. There appear to be multiple theories, cognitive and behavioral techniques that have been studied in applied settings [4].

The use of positive affect as a process or adjunctive treatment is an area that may have value. Positive affect can be induced through an external influence. The topic or issue that is the target of the influence must be viewed as neutral or in a positive perspective for the induction to be effective. Positive affect has not been proven to reverse a negative emotion or feeling. Positive affect appears to increase the positive valence when the issue of interest is viewed as positive or at a minimum neutral $[5,6]$. This specificity of effect is a key aspect of positive affect. This specificity is not a mood shift that acts as a consistent bias, but a trigger than only effects certain issues [7]. Emotions and cognitions appear to be bidirectional causes and effects, based upon the trigger event. That is, an emotion can directly influence the cognitive process or the cognitive evaluation of a situation or, conversely, the environment can result in a changed emotional state [6].

Positive affect is subject to biorhythms related to typical waking patterns in the day. Positive affect parallels the circadian rhythm of body temperature during the day. Positive affect has been measured to begin increasing one hour post waking and reach an approximate steady state approximately three hours later, peaking at the eight-hour mark. The level of positive affect slowly degrades from 
the eight-hour peak, experiencing an acute drop at the 12-hour post waking point. Positive affect, sleep, depression and other mood disorders all appear to be influenced by sleep patterns and energy exertion in daily cycles. Negative affect has not been observed to follow any cyclical or circadian patterns [8].

There are various types of positive affect induction that include imagery, audiotapes, humorous videotapes, video clips, reflection on positive scenarios, autobiographical recall of positive events, fragrance, personal diary of positive events, candy, positive word list/association $[9,10]$. The most important factor in consideration of introducing positive affect, is the amount of time or latency, an individual is influenced rather than the intensity of the affect [9]. Studies previously cited constitute controlled laboratory studies that have not been tested in an applied setting.

The evaluation of positive affect inductions in applied settings across multiple days may be influenced by factors not previously identified. As an example, there is a definite circadian rhythm to levels of positive affect. After rising from sleep, positive affect increases and maintains levels throughout the day, peaks at approximately 8-hours post waking, and then decreases in the evening $[8,11]$.

\section{Research Need and Problems}

The research implications and opportunities to explore the impact of positive affect on health care decisions and behavior appears to be numerous. Meller's and McGraw's subjective expected pleasure theory has not been applied to health and medical decision making situations [12]. The role of emotions has 
not been applied to personal decisions regarding medical care and preventive care [13], and is recommended for additional study of nutritional intake [14]. It appears that depressed affect supports indecisiveness, yet the impact of this circumstance on health related decisions has not been directly explored [15]. The effects of specific emotions such as anger, sadness and fear has not been reported [16]. It appears that evidence is absent regarding the effect of positive affect on coping behavior and health behaviors [9].

The literature reviewed appears to be missing several facets of positive affect study. Practical application of induction techniques outside of controlled conditions has not been reported. A multi-week correlation study of selfreported emotions has been published [14], but no prospective randomized experimental study of positive affect was found.

\section{Conclusions}

The qualitative investigation results of the exploratory study provided insights and practical solutions for use in the main study. The library of messages was distilled into 24 messages in each of three categories. Elaboration on themes and common traits of messages increased the confidence and strength of the selected messages. Practical suggestions to use telephone text messages (SMS) and email will improve the ability to distribute the main study messages and expected increase in the message dosage during the positive affect treatment period. This precludes the requirement for an IVR telephone system during the main study and increases the tolerance for daily contact. 
The results and analysis were challenged due to the small number of respondents to a given survey and the large number of missing cases. A thorough analysis of the results was not feasible as a result of the small number of cases available through the two-week study. This lack of power rendered most statistical tests not practical. The sample size determination for the main study estimated a minimum of 40 participants and potentially 65 participants depending on the induction effect. The erosion of study participants who agreed to informed consent and were consistent participants in study activities was approximately $50 \%$. The pool of volunteers accepted through informed consent will likely require 80 to 130 participants in each study arm.

The inability to adequately answer the research questions and hypothesis degrades the confidence to implement the main study. The daily PANAS scores may not display adequate specificity. The applied nature of the reporting does not allow for respondents to respond at the same time of the day. Late respondents may introduce a confounding factor that is not easily controlled. Consideration of limiting the PANAS collection to the 7-day recall and eliminating the daily scores is practical when reviewing the exploratory study results.

An unexpected result was the reported negative PANAS scores. Negative affect PANAS scores are collected to monitor unexpected emotional induction or bias. The limited data set precluded any statistically significant conclusions. There was a trend for the negative PANAS scores to decrease during the treatment week. This warrants exploration during the main study. 
The exploratory study was designed to test several aspects of positive affect induction in an applied setting. The induction techniques were explored using qualitative research methods and yielded several insights. These feedback and survey sessions established a practical approach to attempted positive affect inducements.

The measurement of daily PANAS scores proved cumbersome and resulted in sporadic participation in approximately $50 \%$ of the population on any given day. The degraded statistical power through low participation rendered virtually all of the statistical analysis inconclusive.

\section{Discussion}

The completion of the survey instruments did not reveal any negative comments or experiences with the exception of the physical activity recall survey. The completion of the seven-day physical activity recall was the most challenging survey. Participants indicated a degree of frustration over an inability to recall the time and duration of activity for the previous 7-days. The design and distribution of a log-sheet for hand completion prior to the on-line survey application data entry mitigated some of the stated frustration among participants.

The use of the accelerometers was executed without difficulty. Missing data on days when participants temporarily lost the device, or forget to wear the device were noted. The missing data fields were few and did not jeopardize the data analysis power. Participants commented on the novelty of the accelerometer and enjoyed the use of the device during the study. 
The distribution of messages through the various communication channels was completed without any major obstacles. The email and text distribution of messages were the least difficult to design and deploy. The design and programming required for the operation of the automated interactive phone system required a large amount of resources. The time to develop the architecture and program the computer-generated system exceeded 3-months. The ability to connect with participants at each contact exceeded $90 \%$. The timing of the message receipt was not consistent. Participants were unavailable for phone or email contact and did not always receive messages at the target delivery times. The use of multiple channels appears for scheduled contact provided flexibility for participants to select the most convenient communication medium.

The selection of humor messages through the qualitative research process and the subsequent use of the same messages on participants during the treatment phase may have degraded the impact of the humor induction. Participants commented that they considered the humor funny and impactful despite previous exposure was collected anecdotally.

The completion of surveys and the general participation rate of study activities was lower than projected. Generally, approximately $50 \%$ of the participant group did not participant in any given survey or message receipt. Modified protocol approaches may improve this participation rate, but the washout / erosion rate of $50 \%$ is prudent for main study planning. 
The novel delivery of the positive affect inductions appears to have merit and was well tolerated by participants. Increased access to multiple communication venues may improve the timely delivery of induction messages.

The temporal relationship between induction and PANAS score increases and the residual effect of the inductions is not informed from available literature. A longer set of observed daily PANAS scores may allow for improved analysis and conclusions in the main study.

\section{Recommendation}

Further study of positive affect in applied settings appears worthy of resources and investigation. The exploratory study reviewed here documented promising approaches to applied techniques of affect induction. Many challenges were identified in the scientific exploration process that will require subsequent studies to replicate and conquer.

The small number of participants degraded the statistical power of the analysis. The study conclusions could not prove that the positive affect inductions were successful. This may or may not be influenced by the low power of the analysis. Increased study cohorts with a planned wash-out rate of $50 \%$ appears practical to overcome this issue.

The daily variations and external confounding factors such as weather, social issues, news may introduce uncontrolled bias into PANAS scores. The exclusion of daily PANAS surveys and reliance on the seven-day recall may reduce participant wash-out. The seven-day recall displays a more stable level 
of score reproducibility and may be more suitable for the weekly treatment periods.

The exploratory study revealed the promise of measuring the influence of positive affect inductions in an applied setting. Although the findings are inconclusive it appears that there is some level of probability that humor induction techniques will influence positive feelings. A follow-on study with larger cohorts may still not be able to conclude with statistical significance the effect of the inductions, but such a study appears to have merit and advance the scientific knowledge in this field of study 


\section{Exploratory Study References}

1. USDHHS, U.S.D.o.H.a.H.S., Healthy People 2010, P.H.S. U. S. Department of Health and Humana Services, Office of the Surgeon General, Editor. 2000, U.S. Government Printing Office: Washington DC. p. 60.

2. Finkelstein, E.A., I.C. Fiebelkorn, and G. Wang, National medical spending attributable to overweight and obesity: How much, and who's paying? Health Affairs, 2003. Supplement (W3): p. 7.

3. Schroeder, S., We can do better - improving the health of the american people. New England Journal of Medicine, 2007. 357: p. 8.

4. Glanz, K., B.K. Rimer, and F.M. Lewis, Health behavior and health education: theory, research and practice. Third ed. 2002, San Francisco, California: John Wiley \& Sons. 571.

5. Isen, A.M., A role for neuropsychology in understanding the facilitating influence of positive affect on social behavior and cognitive processes, in Handbook of Positive Psychology, C.R. Snyder and S.J. Lopez, Editors. 2005, Oxford Publishing: New York. p. 12.

6. Isen, A.M., Some ways in which positive affect influences decision making and problem solving, in Handbook of Emotions, M. Lewis, J.M. Haviland-Jones, and L.F. Barrett, Editors. 2008, The Guilford Press: New York. p. 36.

7. Isen, A.M., Positive Affect, in Handbook of cognition and emotion, T. Dalgleish and M. Power, Editors. 1999, John Wiley \& Sons: West Sussex. p. 19.

8. Watson, D., et al., The two general activation systems of affect: Structural findings, evolutionary considerations, and psychobiological evidence. Journal of Personality and Social Psychology, 1999. 76(5): p. 19.

9. Lyubomirsky, S., L. King, and E. Diener, The benefits of frequent positive affect: Does happiness lead to success? Psychological Bulletin, 2005. 131(6): p. 52.

10. Isen, A.M. and A. Erez, Some measurement issues in the study of affect, in Oxford Handbook of Methods in Positive Psychology, A.D. Ong and M.H.M.v. Dulmen, Editors. 2007, Oxford University Press.

11. Lawton, R., M. Conner, and R. McEachan, Desire or reason: predicting health behaviors from affective and cognitive attitudes. Health Psychology, 2009. 28(1): p. 10.

12. Mellers, B.A. and A.P. McGraw, Anticipated emotions as guides to choice. Current Directions in Psychological Science, 2001. 10(6): p. 5.

13. Diffenbach, M.A., et al., Emotions and Health Behavior: A self-regulation perspective, in Handbook of Emotions, M. Lewis, J.M. Haviland-Jones, and L.F. Barrett, Editors. 2008, The Guilford Press: New York. p. 17.

14. Macht, M. and G. Simons, Emotions and eating everyday life. Appetite, 2000. 35: p. 7.

15. Consedine, N.S., Health-Promoting and health-damaging effects of emotions: The view from developmental functionalism, in Handbook of Emotions, M. Lewis, J.M. Haviland-Jones, and L.F. Barrett, Editors. 2008, The Guilford Press: New York. p. 15.

16. Macht, M., How emotions affect eating: A five-way model. Appetite, 2008. 50; p. 12. 


\section{CURRICULUM VITAE}

Philip A. Smeltzer

4803 Bilandon Road

Louisville KY 40241

502-744-9293

\section{Education and Training}

B.A., Physical Education

Oregon State University

1977

M.S., Physical Education

Canisius College

2000

Ph.D., Public Health \& Information Science

University of Louisville

2011

\section{Professional Memberships}

- American Association of Health Insurance Plans, Obesity Initiative Task

Force Member

(2008-present)

- BCBSA Disease Management Sub-committee, National Medical Management Forum

- Society of Public Health Educators (1996-present)

- Health Care Executive Forum (1999-2006)

- Association of Fundraising Professionals (2001-2004)

- Association Fellow, Public Health Leadership Institute (class of 2002)

- Association for Worksite Health Promotion (1994-2001)

- American Public Health Association (1995-2001)

- New York State Professional Health Educators (1993-1998)

- American College of Sports Medicine (1994-2001)

- National Wellness Institute (1996-2000) 


\section{Community Leadership}

- Health Promotion Consultant, Humana Foundation (2007-2010)

- Advisor, WNED-TV b-Healthy Initiative for Healthy Children and Families (2005-2007)

- Chairman, Community Health Coalition (2003-2005)

- Health and Recreation Committee, Town of Wilson (2004-2006)

- Educational Degree Task Force, National Athletic Training Association, 20052007

- Chairman, Erie County Stabilization Committee, Health Services Subcommittee (2005)

- Steering Committee Member (elected), Joint Women's Health Initiative and Colorectal/Prostate Cancer Coalition (2000-2001)

- Board Member \& Sports Director, Niagara Frontier Officials, Football. (19982006)

- Charter member and Organizing Committee, National Blue Cross Blue Shield Health Promotion Conference (1996-1999)

- Chair, Worksite Health (elected), New York State Professional Health Educators (1997)

- Chairman, Physical Activity Coalition of Erie County (1998-1999)

\section{Peer Reviewed Conference Selections}

Obesity Management: A Health Plan's Perspective. Obesity Congress Conference. Washington DC. October 2009

Consumer Engagement and Behavior Change. Association of Health Insurance Plans, Institute 2009 Conference. San Diego CA. June 2009.

Where's the ROI. Financial Considerations of Health Coaching. World Research Group, Health Coaching Conference. San Diego CA. October 2008.

Preventive Health Messages: A Health Plan's Approach. Center for Disease Control, Health Marketing Conference. Atlanta GA. August 2008.

Humana \& Virgin HealthMiles Financial Incentive Program. Health \& Wealth Summit. Miami FL. September 2008.

Pediatric Obesity and Pediatric Wellness: A Health Plan's Perspective. Teleconference, September 2007.

Wellness Incentives. World Research Group, Incentives and Wellness Conference. Chicago IL July 2007, November 2007 (Teleconference)

Managed Care Organization Asthma Regional Collaboration. Center for Health Care Studies Conference. Atlanta GA. October 2002. 
Obesity Treatment in Managed Care Organizations. American Obesity

Association Conference, Washington DC. August 2000.

Cardiovascular Disease Management. National Wellness Council. National Conference, Stevens Point WI. July 1998.

\section{Publications}

2008-Present Reviewer for American Journal of Health Behavior

Evaluating Health Promotion Programs: Applying Best Practices to Evaluate and Study the Impact of Health Promotion Programs Benefit Costs, Health and Productivity. PA Smeltzer, R Ozminkowski, S Musich. Corporate Wellness Magazine. March 8, 2011.

Are We Having Fun Yet: Influence of Positive Affect. IAWHP Newsletter. May/June 2011.

Well Informed. The WorkCare Group, Inc. 2004. Charlottesville Virginia. Contributing Editor, Philip Smeltzer; Walking: Possibly the Best Exercise, Rating Popular Fitness Activities, Improving Your Flexibility, Stretching for Health, Building Strong Muscles. P 6-15.

The Effectiveness of One-on-One Nurse Education on the Outcomes of HighRisk Adult and Pediatric Asthma Patients. James D. Forshee, MD, MBA. Forshee JD, Whalen EB, Hackel R, Butt LT, Smeltzer PA, Martin J, Lavin P, Buchner DA. Managed Care Interface. 1998;12(11):82 - 92. 